\title{
Numerical infinities and infinitesimals: Methodology, applications, and repercussions on two Hilbert problems
}

\author{
Yaroslav D. Sergeyev*
}

\begin{abstract}
In this survey, a recent computational methodology paying a special attention to the separation of mathematical objects from numeral systems involved in their representation is described. It has been introduced with the intention to allow one to work with infinities and infinitesimals numerically in a unique computational framework in all the situations requiring these notions. The methodology does not contradict Cantor's and non-standard analysis views and is based on the Euclid's Common Notion no. 5 'The whole is greater than the part' applied to all quantities (finite, infinite, and infinitesimal) and to all sets and processes (finite and infinite). The methodology uses a computational device called the Infinity Computer (patented in USA and EU) working numerically (recall that traditional theories work with infinities and infinitesimals only symbolically) with infinite and infinitesimal numbers that can be written in a positional numeral system with an infinite radix. It is argued that numeral systems involved in computations limit our capabilities to compute and lead to ambiguities in theoretical assertions, as well. The introduced methodology gives the possibility to use the same numeral system for measuring infinite sets, working with divergent series, probability, fractals, optimization problems, numerical differentiation, ODEs, etc. (recall that traditionally different numerals $\infty, \aleph_{0}, \omega$, etc. are used in different situations related to infinity). Numerous numerical examples and theoretical illustrations are given. The accuracy of the achieved results is continuously compared with those obtained by traditional tools used to work with infinities and infinitesimals. In particular, it is shown that the new approach allows one to observe mathematical objects involved in the Hypotheses of Continuum and the Riemann zeta function with a higher accuracy than it is done by traditional tools. It is stressed that the hardness of both problems is not related to their nature but is a consequence of the weakness of traditional numeral systems used to study them. It is shown that the introduced methodology and numeral system change our perception of the mathematical objects studied in the two problems.
\end{abstract}

Mathematics Subject Classification (2010). 65-02, 03-02, 11-02, 40-02, 00A30, 03E65, 03E50.

Keywords. Numerical infinities and infinitesimals, numbers and numerals, grossone, Infinity Computer, numerical analysis, infinite sets, divergent series, Continuum Hypothesis, Riemann zeta function, numerical differentiation, ODEs, optimization, probability, fractals.

${ }^{*}$ The author is very grateful to the reviewers for their careful and meticulous reading of the paper.

Yaroslav D. Sergeyev, University of Calabria, Via P. Bucci, Cubo 42-C, 87030 Rende, Italy; Lobachevsky University, Nizhni Novgorod, Russia; Institute of High Performance Computing and Networking of the National Research Council of Italy, Rende, Italy

E-mail: yaro@dimes.unical.it 


\section{Contents}

1 Introduction .................... 3

2 Numeral systems and numbers they can express . . . . . . . . . . 6

2.1 Differences among numeral systems used to express finite and infinite

quantities ..................... 6

2.2 Amazonian and Australian tribes prompt a new point of view on infinity . 7

3 The computational methodology . . . . . . . . . . . . . . . . . . 11

3.1 Methodological postulates . . . . . . . . . . . . . . . . 11

3.2 Counting huge finite quantities in practice - a granary example . . . . 16

4 A new way of counting and the infinite unit of measure (1) . . . . . . . . . 17

4.1 The Infinite Unit Axiom and first examples . . . . . . . . . . . . . . 17

4.2 Postulate 3 versus bijections of infinite sets: no contradiction . . . . . . . 24

4.3 Positional numeral system with the infinite radix (1) . . . . . . . . 27

4.4 Arithmetical operations with (1)-based numerals . . . . . . . . . . 29

5 Numerical infinities and infinitesimals in applications . . . . . . . . . 32

5.1 Higher order numerical differentiation on the Infinity Computer . . . . . . 32

5.2 Infinitesimal perturbations for solving systems of linear equations . . . . 34

5.3 Infinite penalty coefficients in constrained non-linear optimization . . . 37

5.4 Lexicographic multi-objective linear programming . . . . . . . . . . . . 39

5.5 Numerical methods using infinitesimals for solving ODEs . . . . . . . . 40

6 Traditional mathematical objects viewed from the new methodological positions 44

6.1 Infinite sequences and Turing machines . . . . . . . . . . . . . 44

6.2 From divergent series to sums with a fixed infinite number of addends . . 47

6.3 Riemann series theorem and Ramanujan summation . . . . . . . . . . 51

6.4 From limits to expressions evaluated at actual infinite/infinitesimal points 55

6.5 Functions, continuity, derivatives, and integrals . . . . . . . . . . 59

7 Measuring infinite sets and the Continuum Hypothesis . . . . . . . . . . . 65

7.1 Measuring infinite sets with elements defined by formulae . . . . . . 65

7.2 Countable sets can be measured more accurately . . . . . . . . . 67

7.3 The number of elements of sets having the cardinality of the continuum . 70

7.4 Relations to results of Georg Cantor . . . . . . . . . . . . 72

8 Repercussions on the Riemann zeta function . . . . . . . . . . . . . 76

8.1 Some Euler's results revisited in the (1)-based framework . . . . . . . 78

8.2 Calculating $\zeta(s, n)$ for different infinite values of $n$ at $s=0,-1,-2$, etc . . 83

9 Grossone for studying probability and fractals . . . . . . . . . . . 87

9.1 Probability and absence of nonempty sets of measure zero . . . . . . . 87

9.2 Koch snowflake and its quantitative analysis using (1)-based numerals . . . 89

10 A brief conclusion . . . . . . . . . . . . . . . . . . . . 93

References . . . . . . . . . . . . . . . . . . . . . . . 94 


\section{Introduction}

In different periods of human history, mathematicians and physicists developed mathematical languages and philosophical positions leading to different approaches to the ideas of infinite and infinitesimal (see [3, 6, 10, 16, 23, 25, 42, 44, 46, 49, 51, 53, 59, 63, 64, $72,77,82,106,111,112,113])$ and references given therein). There is no need to explain the importance of this subject for Mathematics. Let us just mention that the Continuum Hypothesis related to infinity has been included by David Hilbert as the Problem Number One in his famous list of 23 unsolved mathematical problems (see [53]) that have influenced strongly development of Mathematics in the $X X^{\text {th }}$ century. The famous Riemann Hypothesis is the problem number 8 in this list and it is also related to infinity since the Riemann zeta function is introduced as an infinite series.

Many approaches describing manipulations with infinities and infinitesimals are rather old: ancient Greeks following Aristotle distinguished the potential infinity from the actual infinity; John Wallis (see [112]) credited as the person who introduced the infinity symbol, $\infty$, published his work Arithmetica infinitorum in 1655; the foundations of analysis we use nowadays were developed more than 200 years ago with the goal to develop mathematical tools allowing one to solve problems that were emerging in the world at that time; Georg Cantor (see [16]) has introduced his cardinals and ordinals more than 140 years ago, as well. As a result, mathematical languages that we use now to work with infinities and infinitesimals do not reflect numerous achievements made by Physics of the $X X^{\text {th }}$ century ${ }^{1}$. Let us illustrate this observation by a couple of examples.

We know from the modern Physics that the same object can be viewed as either discrete or continuous in dependence on the instrument used for the observation (we see a table continuous when we look at it by eye and we see it discrete (consisting of molecules, atoms, etc.) when we observe it through a microscope). In addition, physicists do not give absolute results of their observations. Together with the result of the observation they always supply the accuracy of the instrument used for this observation.

In Mathematics, both facts are absent: each mathematical object (e.g., function) is either discrete or continuous and nothing is said about the accuracy of the observation of the mathematical objects and about tools used for these observations. The mathematical notion of continuity itself comes from the $X I X^{\text {th }}$ century. Many of mathematical notions have an absolute character and the ideas of relativity are rarely present in them. One of the main ideas of Physics of the $X X^{t h}$ century regarding ideas of the influence of the instrument of an observation on the object of the observation are almost absent in Mathematics, as well.

In some sense, there exists a gap between achievements in Physics made during the last two hundred years (especially during the $X X^{\text {th }}$ century) and their mathematical mod-

${ }^{1}$ Even the brilliant efforts of the creator of the non-standard analysis Robinson that were made in the middle of the $X X^{\text {th }}$ century have been also directed to a reformulation of the classical analysis (i.e., analysis created two hundred years before Robinson) in terms of infinitesimals and not to the creation of a new kind of analysis that would incorporate new achievements of Physics. In fact, he wrote in paragraph 1.1 of his book [82]: 'It is shown in this book that Leibniz's ideas can be fully vindicated and that they lead to a novel and fruitful approach to classical analysis and to many other branches of Mathematics' (italics mine). In fact, classical analysis reformulated using the language of Robinson was then used to address such areas as topology, probability, etc. 
els that continue to be written using the mathematical language developed two centuries ago on the basis of (among other things) physical ideas of that remote time that now are absolutely outdated.

In particular, in relation to the concepts of infinite and infinitesimal we have an analogous situation. In fact, the point of view on infinity accepted nowadays (see [3, 23, 27, $51,58,59,113]$ ) takes its origins from the famous ideas of Cantor (see [16]) who in the second half of the $X I X^{t h}$ century has shown that there exist infinite sets having different cardinalities. Infinitesimals have been developed even earlier when, in the early history of calculus, arguments involving infinitesimals played a pivotal role in the differential calculus developed by Leibniz and Newton (see $[63,77]$ ). At that time the notion of an infinitesimal, however, lacked a precise mathematical definition and in order to provide a more rigorous foundation for the calculus infinitesimals were gradually replaced by the d'Alembert-Cauchy concept of a limit (see [19, 28]).

The creation of a rigorous mathematical theory of infinitesimals on which it would be possible to construct Calculus remained an open problem until the end of the 1950s when, as was already mentioned, Robinson (see [82]) has introduced his non-standard analysis approach (Levi-Civita has proposed his transfinite numbers in [64] much earlier but they have not received so broad dissemination as the approach of Robinson). He has shown that non-archimedean ordered field extensions of the reals contained numbers that could serve as infinitesimals and their reciprocals could serve as infinitely large numbers. Robinson then has derived the theory of limits, and more generally of calculus, and has found a number of important applications of his ideas in many other fields of Mathematics (see [82]). It is important to emphasize that in his approach Robinson used Cantor's mathematical tools and terminology (cardinal numbers, countable sets, continuum, oneto-one correspondence, etc.) thus incorporating advantages and disadvantages of Cantor's approach into non-standard analysis.

After these few introductory words let us define the aim of the present text. The first goal of this paper is to bridge the gap between modern Physics and Mathematics at least partially, insofar as it concerns the separation of an object and a tool used to observe it existing in Physics and often not present in Mathematics. The main attention will be dedicated to infinities and infinitesimals and ways of their representation in comparison with representation of finite numbers. Recall that the same numerals are used to represent finite numbers in all the occasions we need them, in contrast, different infinities are used in different situations. Traditionally, these various kinds of infinity are perceived by people as distinct mathematical objects. The approach developed here shows that this is not the case. It argues that the objects - infinite numbers - are the same, they are just viewed in different ways by different theories. For example, in analysis and set theory, $\infty$ and $\aleph_{0}$ are not two different objects, they are two different images of infinite quantities that people see through different 'lenses' provided by analysis and set theory.

Then, the second goal of the paper is to describe a numeral system expressing infinities and infinitesimals that can be used in all situations where we need to work with them. This is done in order to have a situation similar to what we have with finite numbers where numerals expressing them can be used in all the occasions we need finite quantities. Several examples of the usage of the new numerals expressing infinities and infinitesimals are given to illustrate their versatility. In particular, numerous numerical applications and 
the two Hilbert problems mentioned above will be considered.

Finally, the third goal consists of describing a computational methodology using the introduced numeral system and a computational device called the Infinity Computer (patented in USA and EU, see [92]) working numerically ${ }^{2}$ with finite, infinite, and infinitesimal numbers in a unique computational framework and in accordance with Euclid's Common Notion no. 5 'The whole is greater than the part'. Several numerical algorithms and applications are described and discussed.

It should be emphasized immediately that the approach to be presented here and originated from $[86,88,94,99,101]$ is not a contraposition to the ideas of Cantor, Levi-Civita, and Robinson. In contrast, it is introduced as an applied evolution of their ideas. The new computational methodology introduces the notion of the accuracy of numeral systems and shows that different numeral systems can express different sets of finite and infinite numbers with different accuracies. It can be shown that Cantor's alephs and numerals described here have different accuracies and cases where the new tools are more accurate can be provided. Thus, traditional approaches and the methodology described here do not contradict one another, they are just different instruments having different accuracies for observations of mathematical objects.

A number of papers studying connections of the approach discussed here to the historical panorama of ideas dealing with infinities and infinitesimals (see [65, 66, 67, 75, 103]) have been published. In particular, metamathematical investigations on the new theory and its consistency can be found in [66]. The methodology described here has been successfully applied in several areas of Mathematics and Computer Science: single and multiple criteria optimization (see [20, 32, 33, 34, 43, 116]), cellular automata (see [29, 30, 31]), Euclidean and hyperbolic geometry (see [68, 69]), percolation (see $[56,57,110]$ ), fractals (see $[15,87,89,97,102,110]$ ), infinite series and the Riemann zeta function (see [91, 96, 99, 101, 114]), the first Hilbert problem, Turing machines, and supertasks (see [81, 93, 103, 104]), numerical differentiation and numerical solution of ordinary differential equations (see $[2,74,95,98,105])$, etc. Some of these applications will be discussed in the following pages.

The survey organizes the presentation of existing results in a natural progression, moving from first principles to more and more sophisticated applications. A special attention is devoted to the introduction of numerical methods that do away with the traditional resort to iterative procedures (e.g., in the computation of derivatives or the resolution of linear and non-linear optimization problems and ODEs) and the treatment of the Continuum Hypothesis and the Riemann zeta function. The style of presentation was especially designed to invite contributions to the existing literature from interested researchers.

The rest of the survey is structured as follows. Section 2 discusses the importance of numeral systems used to express numbers for mathematical theory and practice. Section 3 describes the computational methodology that allows one to look at infinities and infinitesimals (and Mathematics, in general) in a new way that is closer to modern Physics than traditional mathematical views. An infinite unit of measure that is used as the radix of a positional numeral system is introduced in Section 4 showing also that this numeral

\footnotetext{
${ }^{2}$ Recall that numerical computations work with approximate floating point numbers, while symbolic computations are the exact manipulations with mathematical expressions containing variables that have not any given value and are thus manipulated as symbols.
} 
system gives a possibility to express finite, infinite, and infinitesimal numbers in a unique framework and to execute numerical (not symbolic) operations with all of them. Section 5 discusses a variety of numerical applications where the new methodology can be useful. Traditional mathematical objects viewed from the new methodological positions are studied in Section 6 whereas Section 7 establishes relations of the new methodology to some of results of Cantor and the Continuum Hypothesis. Repercussions of the new methodology on the Riemann zeta function are discussed in Section 8. Examples related to probability and fractals are given in Section 9. Finally, Section 10 concludes the survey.

\section{Numeral systems and numbers they can express}

2.1. Differences among numeral systems used to express finite and infinite quantities. Let us start by recalling that a numeral is a symbol (or a group of symbols) that represents a number that is a concept. The same number can be represented by different numerals. For example, symbols '10', 'ten', 'IIIIIIIIII' 'X', '=', and 'Ĩ' are different numerals, but they all represent the same number ${ }^{3}$. Rules used to write down numerals together with algorithms for executing arithmetical operations form a numeral system. Thus, numbers can be considered as objects of an observation that are represented (observed) by instruments of the observation, i.e., by numerals and, more general, by numeral systems.

In our everyday activities with finite numbers the same finite numerals are used for different purposes (e.g., the same numeral 10 can be used to express the number of elements of a set, to indicate the position of an element in a sequence, and to execute practical computations). In contrast, when we need to work with infinities and/or infinitesimals, the situation changes drastically and we face a number of distinctions and complications.

First, different numerals are used to work with infinities and infinitesimals in different situations. To illustrate this fact it is sufficient to mention that we use the symbol $\infty$ in standard analysis, symbol $\omega$ for working with ordinals, symbols $\aleph_{0}, \boldsymbol{\aleph}_{1}, \ldots$ for dealing with cardinalities, etc.

Second, the mainstream of the traditional Mathematics very often does not pay a great attention to the distinction between numbers and numerals (in this occasion it is necessary to recall constructivists who studied this issue, see, e.g., [71]). As a result, many theories dealing with infinite and infinitesimal quantities have a symbolic (not numerical) character (see Footnote 2). For instance, non-standard models and non-archimedean fields use either a generic infinite number or a generic infinitesimal in their constructions (e.g., Levi-Civita numbers are built using a generic infinitesimal $\varepsilon$ ) whereas our numerical

\footnotetext{
${ }^{3}$ The last two numerals, = and $\tilde{I}$, are probably less known. The former belongs to the Maya numeral system where one horizontal line indicates five and two lines one above the other indicate ten. Dots are added above the lines to represent additional units. For instance, numerals $=$ and $\doteq$ mean, respectively, ten and eleven in this numeral system. The latter symbol, I, belongs to the Cyrillic numeral system derived from the Cyrillic script. This numeral system was developed in the late $X^{\text {th }}$ century and was used by South and East Slavic peoples. The system was used in Russia as late as the early $X V I I I^{\text {th }}$ century when it was replaced with Arabic numerals. To distinguish numbers from text a special sign, ${ }^{\sim}$, called titlo is drawn over the symbols showing so that this is a numeral and, therefore, it represents a number and not just a character of text.
} 
computations with finite quantities are concrete and not generic. If we consider a finite $n$ then different values can be assigned to it, e.g., we can use the numeral 34 and write $n=34$ (clearly, any other known numeral used to express finite quantities and consisting of a finite number of symbols can be taken for this purpose). Notice that the finiteness of the number of symbols in the numeral is necessary for executing practical computations since we should be able to write down (and/or to store) values we execute operations with.

In contrast, if we consider a non-standard infinite $m$ then it is not clear which numerals consisting of a finite number of symbols can be used to assign a concrete value to $m$ since non-standard analysis does not provide numeral systems that can be used for this purpose. For a generic infinitesimal $\varepsilon$ again it is not clear which numerals can be used to assign a value to $\varepsilon$ and to write $\varepsilon=\ldots$. Moreover, approaches of this kind leave unclear such issues as, e.g., whether the infinite $1 / \varepsilon$ is integer or not or whether $1 / \varepsilon$ is the number of elements of a concrete infinite set. If one wishes to consider two infinitesimals (or infinities) $h_{1}$ and $h_{2}$, where $h_{2}$ is not expressed in terms of $h_{1}$, then it is not clear how to compare them because numeral systems that can express different values of infinities and infinitesimals are not provided by this kind of techniques. In contrast, when we work with finite quantities, then we can compare $n$ and $k$ if they assume numerical values, e.g., $k=25$ and $n=78$ then, by using rules of the numeral system the symbols 25 and 78 belong to, we can compute that $n>k$.

Third, many arithmetics used to deal with infinities not only should be used for specific purposes only but in addition they are quite different with respect to the way we execute computations with finite quantities. Let us give some examples:

- There exist undetermined operations $\left(\infty-\infty, \frac{\infty}{\infty}\right.$, etc.) that are absent when we work with finite numbers.

- Arithmetic with ordinals is very different from arithmetic with finite quantities. For instance, addition and multiplication are not commutative (e.g., $1+\omega=\omega$ and $\omega+1>\omega)$, there does not exist an ordinal $\gamma$ such that $\gamma+1=\omega$, etc.

- Arithmetic with $\infty$ and infinite cardinals does not satisfy Euclid's Common Notion 5 saying 'The whole is greater than the part' that holds when we work with finite quantities. In fact, it follows for these numerals, e.g., $\infty+1=\infty$ and $\boldsymbol{\aleph}_{0}+1=$ $\aleph_{0}$ whereas clearly for any finite $x$ it follows $x+1>x$.

Even though there exist codes allowing one to work symbolically with $\infty$ and other symbols related to the concepts of infinity and infinitesimals, traditional computers work numerically only with finite numbers and situations where the usage of infinite or infinitesimal quantities is required are studied mainly theoretically (see [6, 16, 25, 44, 49, $53,63,64,82,112]$ and references given therein). The fact that numerical computations with infinities and infinitesimals have not been implemented so far on computers can be explained by difficulties mentioned above. There exist also practical troubles that preclude an implementation of numerical computations with infinity and infinitesimals. For example, it is not clear how to store an infinite quantity in a finite computer memory.

2.2. Amazonian and Australian tribes prompt a new point of view on infinity. In order to understand how one can change his/her view on infinity, let us consider some 
numeral systems used to express finite numbers. During the history of humanity numeral systems (see, e.g., $[25,27]$ ) became more and more sophisticated allowing people to express more and more numbers. Since new numeral systems appear very rarely, in each concrete historical period people tend to think that any number can be expressed by the current numeral system and the importance of numeral systems for Mathematics is very often underestimated (especially by pure mathematicians who often write phrases of the kind 'Let us take any $x$ from the interval $[1,2]$ ' and do not specify how this operation of taking can be executed). However, if we observe the situation with expression of numbers in the historical prospective, we can immediately see limitations that various numeral systems induce. It becomes clear that due to practical reasons different numeral systems can express different limited sets of numbers and they can be more or less suitable for executing arithmetical operations.

In fact, all numeral systems have practical limitations on the numbers they can express. Even the powerful positional system is not able to express, e.g., the number $\sqrt{2}$ by a finite number of symbols (remind that the finiteness of symbols in operands is essential for executing numerical computations) and this special numeral, $\sqrt{2}$, is deliberately introduced to express the desired quantity. Moreover, there exists another practical limitation that prohibits us to work even with numbers having a finite number of digits in their positional records. It consists of the fact that nobody is able to write down very long numbers, e.g., a numeral in the decimal positional system having $10^{100}$ digits cannot be written due to physical limitations even by the fastest existing computers. As a consequence, no practical operations can be done with these numbers, no theorems involving them can be formulated, etc.

There exist many numeral systems that are weaker than the positional one. For instance, Roman numeral system is not able to express zero and negative numbers and such expressions as III - VIII or X - X are indeterminate forms in this numeral system. As a result, before the appearance of positional systems and the invention of zero (by the way, the second event was several hundred years later with respect to the first one) mathematicians were not able to create theorems involving zero and negative numbers and to execute computations with them. The appearance of the positional numeral system not only has allowed people to execute new operations but has led two new theoretical results, as well. Thus, numeral systems not only limit us in practical computations, they induce boundaries on theoretical results, as well.

Even though Roman numeral system is weaker than the positional one it is not the weakest numeral system. There exist very weak numeral systems allowing their users to express very few numbers and one of them is illuminating for our study. This numeral system is used by a tribe, Pirahã, living in Amazonia nowadays. A study published in Science in 2004 (see [45]) describes that these people use an extremely simple numeral system for counting: one, two, many. For Pirahã, all quantities larger than two are just 'many' and such operations as $2+2$ and $2+1$ give the same result, i.e., 'many'. Using their weak numeral system Pirahã are not able to see, for instance, numbers 3,4 , and 5 , to execute arithmetical operations with them, and, in general, to say anything about these numbers because in their language there are neither words nor concepts for that.

It is worthy of mention that the result 'many' is not wrong. It is just inaccurate. Analogously, when we observe a garden with 517 trees, then both phrases: 'There are 
517 trees in the garden' and 'There are many trees in the garden' are correct. However, the accuracy of the former phrase is higher than the accuracy of the latter one. Thus, the introduction of a numeral system having numerals for expressing numbers 3 and 4 leads to a higher accuracy of computations and allows one to distinguish results of operations $2+1$ and $2+2$.

The poverty of the numeral system of Pirahã leads also to the following results

$$
\text { 'many' }+1=\text { 'many', 'many’ }+2=\text { 'many’, 'many’ + 'many’ = 'many' }
$$

that are crucial for changing our outlook on infinity. In fact, by changing in these relations 'many' with $\infty$ we get relations used to work with infinity in the traditional calculus

$$
\infty+1=\infty, \quad \infty+2=\infty, \quad \infty+\infty=\infty .
$$

Analogously, if we consider Cantor's cardinals (where, as usual, numeral $\boldsymbol{\aleph}_{0}$ is used for cardinality of denumerable sets and numeral $c$ for cardinality of the continuum, see $[16,22,25,53,64,82,113])$ we have similar relations

$$
\begin{gathered}
\mathfrak{\aleph}_{0}+1=\mathfrak{\aleph}_{0}, \quad \mathfrak{\aleph}_{0}+2=\mathfrak{\aleph}_{0}, \quad \mathfrak{\aleph}_{0}+\mathfrak{\aleph}_{0}=\mathfrak{\aleph}_{0}, \\
\mathfrak{c}+1=\mathfrak{c}, \quad \mathfrak{c}+2=\mathfrak{c}, \quad \mathfrak{c}+\mathfrak{c}=\mathfrak{c} .
\end{gathered}
$$

It should be mentioned that the astonishing numeral system of Pirahã is not an isolated example of this way of counting. In fact, the same counting system, one, two, many, is used by the Warlpiri people, aborigines living in the Northern Territory of Australia (see [14]). The Pitjantjatjara people living in the Central Australian desert use numerals one, two, three, big mob (see [62]) where 'big mob' works as 'many'. It makes sense to remind also another Amazonian tribe - Mundurukú (see [79]) who fail in exact arithmetic with numbers larger than 5 but are able to compare and add large approximate numbers that are far beyond their naming range. Particularly, they use the words 'some, not many' and 'many, really many' to distinguish two types of large numbers. Their arithmetic with 'some, not many' and 'many, really many' reminds one strongly of the rules Cantor uses to work with $\aleph_{0}$ and $\mathfrak{c}$, respectively. In fact, it is sufficient to compare

$$
\text { 'some, not many'+ 'many, really many' = 'many, really many' }
$$

with

$$
\aleph_{0}+\mathfrak{c}=\mathfrak{c}
$$

to see this similarity.

Let us compare now the weak numeral systems involved in (2.1), (2.5) and numeral systems used to work with infinity. We have already seen that relations (2.1) are results of the weakness of the numeral system employed. Moreover, the usage of a stronger numeral system shows that it is possible to pass from records $1+2=$ 'many' and $2+2=$ 'many' providing for two different expressions the same result, i.e., 'many', to more precise answers $1+2=3$ and $2+2=4$ and to see that $3 \neq 4$. In these examples we have the same objects small finite numbers - but results of computations we execute are different in dependence 
of the instrument - numeral system - used to represent numbers. Substitution of the numeral 'many' by a variety of numerals representing numbers 3 , 4 , etc. allows us both to avoid relations of the type (2.1), (2.5) and to increase the accuracy of computations.

Relations (2.2)-(2.4), (2.6) manifest a complete analogy with (2.1), (2.5). Canonically, symbols $\infty, \aleph_{0}$, and $\mathrm{c}$ are identified with concrete mathematical objects and (2.2)(2.4), (2.6) are considered as intrinsic properties of these infinite objects (see e.g., [16, $22,25,53,64,82,113])$. However, the analogy with (2.1), (2.5) shows that relations (2.2)-(2.4), (2.6) do not reflect the nature of infinite objects. They are just a result of weak numeral systems used to express infinite quantities. As (2.1), (2.5) show the lack of numerals in numeral systems of Pirahã, Warlpiri, Pitjantjatjara, and Mundurukú for expressing different finite quantities, relations (2.2)-(2.4), (2.6) show shortage of numerals in mathematical analysis and in Cantor's theories for expressing different infinite numbers. Another hint leading to the same conclusion is the situation with indeterminate forms of the kind III-V in Roman numerals that have been excluded from the practice of computations after introducing positional numeral systems.

Thus, the analysis made above allows us to formulate the following key observation that changes our perception of infinity:

\section{Our difficulty in working with infinity is not a consequence of the nature of infinity but is a result of weak numeral systems having too little numerals to express the multitude of infinite numbers.}

The way of reasoning where the object of the study is separated from the tool used by the investigator is very common in natural sciences where researchers use tools to describe the object of their study and the used instrument influences the results of the observations and determine their accuracy. The same happens in Mathematics studying natural phenomena, numbers, objects that can be constructed by using numbers, sets, etc. Numeral systems used to express numbers are among the instruments of observation used by mathematicians. As we have illustrated above, the usage of powerful numeral systems gives the possibility to obtain more precise results in Mathematics in the same way as usage of a good microscope gives the possibility of obtaining more precise results in Physics. Traditional numeral systems have been developed to express finite quantities and they simply have no sufficiently high number of numerals to express different infinities (and infinitesimals).

In order to avoid situations of the type (2.2)-(2.4), (2.6), a numeral system allowing one to express a variety of different infinities and infinitesimals has been introduced recently in $[86,88,94,99,101]$. The respective computational methodology allows one to execute numerical computations with infinities and infinitesimals on the Infinity Computer (USA patent 7,860,914, EU patent 1728149). This numeral system uses the same numerals for different purposes for dealing with infinities and infinitesimals as it happens with finite numerals that can be used in all the occasions where we need to work with finite quantities. 


\section{The computational methodology}

3.1. Methodological postulates. The aim of this section is to introduce a computational methodology that would allow one to work with infinite and infinitesimal quantities in the same way as one works with finite numbers. Evidently, it becomes necessary to define what does it mean in the same way. Usually, in modern Mathematics, when it is necessary to define a concept or an object, logicians try to introduce a number of axioms describing the object. However, this way is fraught with danger because of the following reasons. First of all, when we describe a mathematical object or concept we are limited by the expressive capacity of the language we use to make this description. A more rich language allows us to say more about the object and a weaker language - less (remind Pirahã that are not able to say a word about number 4). Thus, development of the mathematical (and not only mathematical) languages leads to a continuous necessity of a transcription and specification of axiomatic systems. Second, there is no guarantee that the chosen axiomatic system defines 'sufficiently well' the required concept and a continuous comparison with practice is required in order to check the goodness of the accepted set of axioms. However, there cannot be again any guarantee that the new version will be the last and definitive one.

In this survey, we describe a different, significantly more applied and less ambitious view on axiomatic systems related only to utilitarian necessities to make computations. In contrast to the modern mathematical fashion that tries to make all axiomatic systems more and more precise (decreasing so degrees of freedom of the studied part of Mathematics), we just define a set of general rules describing how practical computations should be executed leaving as much space as possible for further changes and developments dictated by practice of the introduced mathematical language. Notice also that historically human beings started to count without any help of axiomatic systems (and nowadays children learn to count by practice without studying, e.g., Peano axioms). The spirit of this text is the same, namely, it shows how to count and gives examples where this way of counting is useful and more precise with respect to traditional systems of counting. Speaking metaphorically, we prefer to make a hammer and to use it instead of describing what a hammer is and how it works. Moreover, as will become clear soon, the methodological postulates we are to introduce will follow Physics and state that an 'absolute' or 'final' definition of a hammer cannot be given.

We start by introducing three postulates that will fix our methodological positions with respect to infinite and infinitesimal quantities and Mathematics, in general. Notice, that methodological postulates will be stated in this section and their concrete implementation will be carried out in the subsequent section that will introduce practical rules required to execute computations that should be used together with the Postulates both in practical computations and theoretical investigations.

After this preliminary discussion let us start to introduce the first postulate. Usually, when mathematicians deal with infinite objects (sets or processes) it is supposed that human beings are able to execute certain operations infinitely many times. For example, in a fixed numeral system it is possible to write down a numeral with any number of digits. However, this supposition is an abstraction because we live in a finite world and all human beings and/or computers finish operations they have started. In the methodology 
to be introduced, this abstraction is not used and the following postulate is adopted.

Methodological Postulate 1. We postulate existence of infinite and infinitesimal objects but accept that human beings and machines are able to execute only a finite number of operations.

Thus, we accept that we shall never be able to give a complete (in any sense) description of infinite objects due to our finite capabilities. Particularly, this means that we accept that we are able to write down only a finite number of symbols to express numbers. However, we do not agre with finitists who deny infinite mathematical objects. We accept their existence and shall try to study them using our finite capabilities.

The second postulate is adopted following the way of reasoning used in natural sciences where researchers use tools to describe the object of their study and the used instrument influences the results of the observations. When a physicist uses a weak lens $A$ and sees two black dots in his/her microscope he/she does not say: The object of the observation is two black dots. The physicist is obliged to say: the lens used in the microscope allows us to see two black dots and it is not possible to say anything more about the nature of the object of the observation until we replace the instrument - the lens or the microscope itself - with a more precise one. Suppose that he/she changes the lens and uses a stronger lens $B$ and is able to observe that the object of the observation is viewed as ten (smaller) black dots. Thus, we have two different answers: (i) the object is viewed as two dots if the lens $A$ is used; (ii) the object is viewed as ten dots by applying the lens $B$. Which of the answers is correct? Both. Both answers are correct but with the different accuracies that depend on the lens used for the observation. The answers are not in opposition one to another, they both describe the reality (or whatever is behind the microscope) correctly with the precision of the used lens. In both cases our physicist discusses what he/she observes and does not pretend to say what the object $i s$.

The same happens in Mathematics studying natural phenomena, numbers, and objects that can be constructed by using numbers. Numeral systems used to express numbers are among the instruments of observations used by mathematicians. The usage of powerful numeral systems gives the possibility to obtain more precise results in Mathematics in the same way as usage of a good microscope gives the possibility of obtaining more precise results in Physics. However, even for the best existing tool the capabilities of this tool will be always limited due to Postulate 1 (we are able to write down only a finite number of symbols when we wish to describe a mathematical object) and due to Postulate 2 we shall never tell, what is, for example, a number but shall just observe it through numerals expressible in a chosen numeral system.

Methodological Postulate 2. We shall not tell what are the mathematical objects we deal with; we just shall construct more powerful tools that will allow us to improve our capacities to observe and to describe properties of mathematical objects.

This Postulate is important for our study because it emphasizes that the triad - object of a study, instrument of a the study, and a researcher executing the study - introduced in Physics in $X X^{\text {th }}$ century exists in Mathematics, as well. With this Postulate we stress that mathematical results are not absolute, they depend on mathematical languages used to formulate them, i.e., there always exists an accuracy of the description of a mathematical result, fact, object, etc. imposed by the mathematical language used to formulate this 
result. For instance, the result of Pirahã $2+2=$ 'many' is not wrong, it is just inaccurate. The introduction of a stronger tool (in this case, a numeral system that contains a numeral for a representation of the number four) allows us to have a more precise answer.

The concept of the accuracy allows us to look at paradoxes in a new way: paradox is a situation where the accuracy of the used language is not sufficient to describe the phenomenon we are interested in. For instance, the answers of Pirahã $2+1=$ 'many' and $2+2=$ 'many' can be viewed as a paradox because from these two records one could conclude that $2+1=2+2$. This paradox shows us the borderline that separates the zone where the language has the high precision from the region where the language cannot be applied because it does not allow one to distinguish different objects within 'many'. Analogously, the records 'many' $+1=$ 'many', $\infty+1=\infty, 1+\omega=\omega \neq \omega+1$, etc. (see (2.1)-(2.6)) can also be viewed as situations where the accuracy of the used numeral systems is not sufficient.

It is necessary to comment upon another important aspect of the distinction between a mathematical object and a mathematical tool used to observe this object. Postulates 1 and 2 impose us to think always about the possibility to execute a mathematical operation by applying a numeral system. They tell us that there always exist situations where we are not able to express the result of an operation. Let us consider, for example, the operation of construction of the successor familiar from number and set theories. In the traditional Mathematics, the question whether this operation can be executed or not is not taken into consideration, it is supposed that it is always possible starting from any integer $n$ to execute the operation $n+1$ and to assign the obtained result to $k$ getting $k=n+1$. Thus, traditionally the problem of the distinction between the existence of the number $k$ and the possibility to execute the operation $n+1$ and to express its result (i.e., to have a numeral that can express $k$ ) is not debated.

Postulates 1 and 2 emphasize this distinction and tell us that: (i) in order to execute the operation it is necessary to have a numeral system allowing one to express both numbers, $n$ and $k$; (ii) for any numeral system there always exists a number $k^{*}$ that cannot be expressed in it. For instance, for Pirahã $k^{*}=3$, for Mundurukú $k^{*}=6$. Even for modern powerful numeral systems there exist such a number $k^{*}$, e.g., as was already mentioned, nobody is able to write down a numeral in the decimal positional system having $10^{100}$ digits $^{4}$. This means that theoretical considerations including the necessity to execute the operation $n+1$ should provide both a practical way to do this and the required numerals, otherwise an ambiguity arises since it is supposed that this operation can be done but it actually cannot be executed.

Another important issue related to Postulate 2 consists of its applications to axiomatic systems. Traditionally, it is supposed that they define some mathematical objects. Due to Postulate 2, they do not define mathematical objects but describe them. They determine formal rules for operating with certain numerals reflecting some (not all) properties of the studied mathematical objects using a certain mathematical language $L$. We are aware that the chosen language $L$ has its accuracy and there always can exist a richer language $\tilde{L}$

\footnotetext{
${ }^{4}$ Suppose that one is able to write down one digit in one nanosecond. Then, it will take $10^{91}$ seconds to record all $10^{100}$ digits. Since in one year there are $31556926 \approx 3.2 \cdot 10^{7}$ seconds, $10^{91}$ seconds are approximately $3.2 \cdot 10^{83}$ years. This is a sufficiently long time since it is supposed that the age of the universe is approximately $1.382 \cdot 10^{10}$ years.
} 
that would allow us to describe the studied object better. As has already been discussed above, any language has a limited expressibility, in particular, there always exist situations where the accuracy of the answers expressible in this language is not sufficient. As a consequence, axiom systems can say about an object they describe only those things that the used language allows. A richer language allows one to say more about the object and a weaker language - less. Thus, development of the mathematical (and not only mathematical) languages leads to a continuous necessity of a transcription and specification of axiomatic systems. In fact, the history of Mathematics shows that important results are under a continuous process of re-writing and improvement in order to increase the level of their precision up to the current level of the development of Mathematics.

Numerals that we use to write down numbers, functions, etc. are among our tools of investigation and, as a result, they strongly influence our capabilities to study mathematical objects. This separation (having an evident physical spirit) of mathematical objects from the tools used for their description is crucial for our study but it is used rarely in contemporary Mathematics. In fact, the idea of finding an adequate (absolutely the best) set of axioms for one or another field of Mathematics continues to be among the most attractive goals for many contemporary mathematicians whereas physicists perfectly understand the impossibility of any attempt to give a final description of physical objects due to limitations of the instruments of study. In Philosophy and Linguistics, the relativity of the language (the instrument) with respect to the world around us (the object of study) is also a well known thing. In Linguistics, it is sufficient to remind the Sapir-Whorf thesis (see $[17,84]$ ), also known as the 'linguistic relativity thesis'. As becomes clear from its name, the thesis does not accept the idea of the universality of language and postulates that the nature of a particular language influences the thought of its speakers. The thesis challenges the possibility of perfectly representing the world with language, because it implies that the mechanisms of any language condition the thoughts of its speakers.

Thus, due to Postulate 2, our point of view on axiomatic systems is significantly more applied with respect to the modern mathematical fashion that tries to make all axiomatic systems more and more precise (decreasing so degrees of freedom of the studied part of Mathematics). We just define a set of general rules describing how practical computations should be executed leaving as much space as possible for further changes and developments dictated by practice of the introduced mathematical language.

For example, from this applied point of view, axioms for real numbers do not define numbers, they describe them. The axioms should be considered together with a particular numeral system $S$ used to write down numerals and are viewed as practical rules (associative and commutative properties of multiplication and addition, distributive property of multiplication over addition, etc.) describing operations with the numerals. The completeness property is interpreted as a possibility to extend $\mathcal{S}$ with additional symbols (e.g., $e, \pi, \sqrt{2}$, etc.) taking care of the fact that the results of computations with these symbols agree with the facts observed in practice. As a rule, assertions regarding numbers that cannot be expressed in a numeral system are avoided ${ }^{5}$. Assertions are made with respect

\footnotetext{
${ }^{5}$ For instance, it is not supposed that real numbers form a field. In fact, in this case real numbers are our objects of study and before saying something we should fix a tool of their observation. To follow Postulate 2 we can discuss sets of real numbers expressible (visible) in a certain fixed numeral system that is our 'lens' in this case.
} 
to what is visible at a mathematical 'lens' and not about the object located behind the lens. This is done again by following Physics where observations are done using different instruments and without specifying the instruments assertions have no meaning. For instance, the question: 'What do you see in this direction?' is meaningless without indication a tool used for the observation. In fact, by eye the observer will see certain things, by microscope other things, by telescope again other things, etc.

Instruments (numeral systems in this case) not only bound our practical capabilities to compute, they can influence theoretical results in Mathematics, as well. We illustrate this statement by considering the following simple phrase 'Let us consider all $x \in[1,2]$ '. For Pirahã, Warlpiri, Pitjantjatjara, and Mundurukú all numbers are just 1 and 2. For people who do not know irrational numbers (or do not accept their existence) all numbers are fractions $\frac{p}{q}$ where $p$ and $q$ can be expressed in a numeral system they know. If both $p$ and $q$ can assume values 1 and 2 (as it happens for Pirahã), all numbers in this case are: $1,1+\frac{1}{2}$, and 2 . For persons knowing positional numeral systems all numbers are those numbers that can be written in a positional system ${ }^{6}$. Thus, in different historical periods (or in different cultures) the phrase 'Let us consider all $x \in[1,2]$ ' has different meanings. As a result, without fixing the numeral system we use to express numbers we cannot fix the numbers we deal with and an ambiguity holds.

Finally, before we switch our attention to Postulate 3, it should be noticed the key difference distinguishing our approach from constructivism. Constructivists assert that it is necessary to construct (in some sense) a mathematical object to prove that it exists. Following the modern Physics, we do not discuss the questions of existence of mathematical objects at all. We discuss just what can be observed through our tools (languages, numeral systems, etc.) and try to improve our tools of the observation.

Let us now introduce the last Postulate. We have already seen in the previous section that situations of the kind (2.2)-(2.4), (2.6) are results of the weakness of numeral systems used to express infinite quantities. Thus, we should treat infinite and infinitesimal numbers in the same manner as we are used to deal with finite ones, i.e., by applying the Euclid's Common Notion no. 5 'The whole is greater than the part'. In our considerations, its straight reformulation 'The part is less than the whole' will be used for practical reasons that will become clear soon. This principle, in our opinion, very well reflects organization of the world around us but is not incorporated in many traditional infinity theories where it is true only for finite numbers. The reason of this traditional discrepancy (as the analysis made in the previous section suggests) is related to the low accuracy of numeral systems used to work with infinity.

Methodological Postulate 3. We adopt the principle 'The part is less than the whole' to all numbers (finite, infinite, and infinitesimal) and to all sets and processes (finite and infinite).

Due to this Postulate, the traditional point of view on infinity accepting such results as $\infty-1=\infty$ should be substituted in a way that $\infty-1<\infty$. One of the motivations in favour of this substitution has already been discussed in detail in connection with the

\footnotetext{
${ }^{6}$ Notice that Cantor's cardinals do not allow us to distinguish the quantity of numbers written in the binary and in the decimal systems providing the same answer: both sets have the cardinality of continuum. This distinction for the new methodological positions will be discussed later.
} 
numerals of Pirahã, Warlpiri, Pitjantjatjara, and Mundurukú. We can introduce another simple argument. Suppose that we are at a point $A$ and at another point, $B$, being infinitely far from $A$, there is an object. Let us see what will happen if we change our position and move, let say, one meter forward in the direction of the point $B$. The traditional numeral system using the symbol $\infty$ will not be able to register this movement in a quantitative way because $\infty-1=\infty$. This numeral system allows us to say only that the point $B$ was infinitely far away before the movement and remains infinitely far away after the movement, i.e., this answer is correct but its accuracy is very low. In practice, due to this traditional way of representing infinity, we are forced to gainsay the finite movement that we have executed. Hereinafter, our goal will be to avoid similar situations by the introduction of a new numeral system that instead of the traditional numerals $\infty, \aleph_{0}, \omega, \aleph_{1}$, etc. is based on a new kind of numerals satisfying Postulates $1-3$ introduced above.

It can seem at first glance that Postulate 3 contradicts Cantor's one-to-one correspondence principle for infinite sets. However, as it will be shown later, this is not the case. Instead, the situation is similar to the example from Physics described above where we have considered two lenses having different accuracies. Analogously, we have two different lenses having different accuracies used to observe the same infinite objects: Cantor's approach and the new one based on Postulates $1-3$.

We conclude this subsection by noticing that the adopted Postulates impose also the style of exposition of results in the survey: we first introduce new mathematical instruments, then show how to use them in several areas of Mathematics giving numerous examples and introducing each item as soon as it becomes indispensable for the problem under consideration.

3.2. Counting huge finite quantities in practice - a granary example. Let us introduce now a way of counting arising in practice where there is the necessity to operate with extremely large finite quantities. This way of computing has existed for millennia but strangely enough has not been studied in depth in Mathematics so far. This example will help us to develop a new intuition required to compute in accordance with Postulates $1-3$ and will be adopted hereinafter to work with infinite quantities.

Imagine that we are in a granary and the owner asks us to count how much grain he has inside it. Obviously, it is possible to answer that there are many seeds in the granary. This answer is correct but its accuracy is low. In order to obtain a more precise answer it would be necessary to count the grain seed by seed but since the granary is huge, it is not possible to do this due to practical reasons.

To overcome this difficulty and to obtain a more precise answer than 'many', people take sacks, fill them with seeds, and count the number of sacks. In this situation, we suppose that: (i) all the seeds have the same measure and all the sacks also; (ii) the number of seeds in each sack is the same and is equal to $K_{1}$ but the sack is so big that we are not able to count how many seeds it contains and to establish the value of $K_{1}$; (iii) in any case the resulting number $K_{1}$ would not be expressible by available numerals.

Then, if the granary is huge and it becomes difficult to count the sacks, then trucks or even big train waggons are used. As it was for the sacks, we suppose that all trucks contain the same number $K_{2}$ of sacks, and all train waggons contain the same number $K_{3}$ 
of trucks, however, the numbers $K_{i}, i=1,2,3$, are so huge that it becomes impossible to determine them. At the end of the counting of this type we obtain a result in the following form: the granary contains 14 waggons, 54 trucks, 18 sacks, and 47 seeds of grain. Note, that if we add, for example, one seed to the granary, we can count it and not only see that the granary has more grain but also quantify the increment. If we take out one waggon, we again are able to say how much grain has been subtracted.

Let us make some considerations upon the way of counting described above. In our example it is necessary to count large quantities. They are finite but it is impossible to count them directly by using an elementary unit of measure, $u_{0}$, (seeds) because the quantities expressed in these units would be too large. Therefore, people are forced to behave as if the quantities were infinite.

To solve the problem of 'infinite' quantities, new units of measure, $u_{1}-$ sacks, $u_{2}-$ trucks, and $u_{3}$ - waggons, are introduced. The new units have an important feature: all the units $u_{i+1}$ contain a certain number $K_{i}$ of units $u_{i}$ but these numbers, $K_{i}, i=1,2,3$, are unknown. Thus, quantities that it was impossible to express using only the initial unit of measure, $u_{0}$, are perfectly expressible in the new units $u_{i}, i=1,2,3$, we have introduced.

Notice that, in spite of the fact that the numbers $K_{i}$ are unknown, the accuracy of the obtained answer is equal to one seed. In fact, if we add one seed we are able to register and to quantify that we have more seeds and if we subtract one waggon and two sacks, we again can quantify the decrease. It is important to emphasize that the decrement can be quantified even if it is required to subtract $m_{i}>n_{i}$ units where $n_{i}$ is the number of units of the type $u_{i}, 0 \leq i \leq 3$, present in the granary. This is done by specifying the number of lacking units $u_{i}$ required to complete the unit $u_{i+1}$, i.e., by allowing the presence of negative values of $n_{i}$. For instance, in the example considered above $n_{2}=54$ and $n_{0}=47$. Subtraction of $m_{2}=56$ trucks and $m_{0}=50$ seeds leads to the following result: 14 train waggons minus 2 trucks and 18 sacks minus 3 seeds.

This key idea of counting by introduction of new units of measure with unknown but fixed values $K_{i}$ will be used in what follows to deal with infinite quantities together with the relaxation allowing one to use negative digits in positional numeral systems.

\section{A new way of counting and the infinite unit of measure (1)}

4.1. The Infinite Unit Axiom and first examples. The way of counting described in the previous section suggests us how to proceed: it is necessary to extend the idea of the introduction of new units of measure from sets and numbers that are huge but finite to infinite sets and numbers. This can be done by extrapolating from finite to infinite the idea that $n$ is the number of elements of the set $\{1,2,3, \ldots, n-1, n\}$.

Thus, the infinite unit of measure is introduced as the number of elements of the set, $\mathbb{N}$, of natural numbers ${ }^{7}$ and expressed by the numeral (1) called grossone. Using the granary example discussed above we can offer the following interpretation: the set $\mathbb{N}$

\footnotetext{
${ }^{7}$ Notice that nowadays not only positive integers but also zero is frequently included in $\mathbb{N}$. However, since historically zero has been invented significantly later than positive integers used for counting objects, zero is not include in $\mathbb{N}$ in this article.
} 
can be considered as a sack and (1) is the number of seeds in the sack. Following our extrapolation, the introduction of (1) allows us to write down the set of natural numbers as $\mathbb{N}=\{1,2,3, \ldots, 1)-1,1\}$. Recall that the usage of a numeral indicating the totality of the elements we deal with is not new in Mathematics. It is sufficient to mention the theory of probability (axioms of Kolmogorov) where events can be defined in two ways. First, as unions of elementary events; second, as a sample space, $\Omega$, of all possible elementary events (or its parts) from which some elementary events have been excluded (or added in case of parts of $\Omega$ ). Naturally, the latter way to define events becomes particularly useful when the sample space consists of infinitely many elementary events.

Grossone is introduced by describing its properties postulated by the Infinite Unit Axiom (IUA) consisting of three parts: Infinity, Identity, and Divisibility. Similarly, in order to pass from natural to integer numbers a new element - zero - is introduced, a numeral to express it is chosen, and its properties are described. The IUA is added to axioms for real numbers (that are considered in sense of Postulate 2). Thus, it is postulated that associative and commutative properties of multiplication and addition, distributive property of multiplication over addition, existence of inverse elements with respect to addition and multiplication hold for grossone as they do for finite numbers ${ }^{8}$.

Let us introduce the axiom and then give some comments upon it. Notice that in the IUA infinite sets will be described in the traditional form, i.e., without indicating the last element. For instance, the set of natural numbers will be written as $\mathbb{N}=\{1,2,3, \ldots\}$ instead of $\mathbb{N}=\{1,2,3, \ldots$, (1) $-1,1\}$. We emphasize that in both cases we deal with the same mathematical object - the set of natural numbers - that is observed through two different instruments. In the first case traditional numeral systems do not allow us to express infinite numbers whereas the numeral system with grossone offers this possibility. Similarly, Pirahã are not able to see finite natural numbers greater than 2 but these numbers (e.g., 3 and 4) belong to $\mathbb{N}$ and are visible if one uses a more powerful numeral system. A detailed discussion will follow shortly to show how to use grossone to calculate and express the number of elements of certain infinite sets.

The Infinite Unit Axiom. The infinite unit of measure is introduced as the number of elements of the set, $\mathbb{N}$, of natural numbers. It is expressed by the numeral (1) called grossone and has the following properties:

Infinity. Any finite natural number $n$ is less than grossone, i.e., $n<$ (1).

Identity. The following relations link (1) to identity elements 0 and 1

$$
0 \cdot(1)=(1) \cdot 0=0, \quad(1)-(1)=0, \quad \frac{1}{(1)}=1, \quad 1^{0}=1, \quad 1^{\oplus}=1, \quad 0^{\circledR}=0 .
$$

Divisibility. For any finite natural number $n$ sets $\mathbb{N}_{k, n}, 1 \leq k \leq n$, being the $n$th parts of the set, $\mathbb{N}$, of natural numbers have the same number of elements indicated by the numeral

\footnotetext{
${ }^{8}$ Remind that we speak about axioms of real numbers in sense of Postulate 2, i.e., axioms define formal rules of operations with numerals in a given numeral system. Therefore, if we want to have a numeral system including grossone, we should fix also a numeral system to express finite numbers. In order to concentrate our attention on properties of grossone, this point will be investigated later.
} 
$\frac{1}{n}$ where

$$
\mathbb{N}_{k, n}=\{k, k+n, k+2 n, k+3 n, \ldots\}, \quad 1 \leq k \leq n, \quad \bigcup_{k=1}^{n} \mathbb{N}_{k, n}=\mathbb{N} .
$$

Let us comment upon this axiom. Its first part - Infinity - is quite clear. In fact, we want to describe an infinite number, thus, it should be larger than any finite number. The second part of the axiom - Identity - tells us that (1) interacts with identity elements 0 and 1 as all other numbers do. In reality, we could even omit this part of the axiom because, due to Postulate 3, all numbers should be treated in the same way and, therefore, at the moment we have stated that grossone is a number, we have fixed the usual properties of numbers, i.e., the properties described in Identity, associative and commutative properties of multiplication and addition, distributive property of multiplication over addition, etc. The third part of the axiom - Divisibility - is the most interesting, since it links infinite numbers to infinite sets (in many traditional theories infinite numbers are introduced algebraically, without any connection to infinite sets) and is based on Postulate 3. Let us first illustrate it by an example.

Example 4.1. If we take $n=1$, then it follows that $\mathbb{N}_{1,1}=\mathbb{N}$ and Divisibility says that the set, $\mathbb{N}$, of natural numbers has (1) elements. If $n=2$, we have two sets $\mathbb{N}_{1,2}$ and $\mathbb{N}_{2,2}$, where

$$
\begin{aligned}
& \mathbb{N}_{1,2}=\left\{\begin{array}{lllll}
1, & 3, & 5, & 7, & \ldots
\end{array}\right\}, \\
& \mathbb{N}_{2,2}=\left\{\begin{array}{llll}
2, & 4, & 6, & \ldots
\end{array}\right\}
\end{aligned}
$$

and they have $\frac{1}{2}$ elements each. Notice that the sets $\mathbb{N}_{1,2}$ and $\mathbb{N}_{2,2}$ have the same number of elements not because they are in a one-to-one correspondence but by the Divisibility axiom. In fact, we are not able to count the number of elements of the sets $\mathbb{N}, \mathbb{N}_{1,2}$, and $\mathbb{N}_{2,2}$ one by one because due to Postulate 1 we are able to execute only a finite number of operations and all these sets are infinite. To define their number of elements we use Divisibility and implement Postulate 3 in practice by determine the number of the elements of the parts using the whole.

Then, if $n=3$, we have three sets

$$
\begin{aligned}
& \mathbb{N}_{1,3}=\{1, \quad 4, \quad 7, \ldots\}, \\
& \mathbb{N}_{2,3}=\left\{\begin{array}{lll}
2, & 5, & \ldots
\end{array}\right\}, \\
& \mathbb{N}_{3,3}=\left\{\begin{array}{llll}
3, & 6, & \ldots
\end{array}\right\}
\end{aligned}
$$

and they have $\frac{1}{3}$ elements each. Note that in formulae (4.3), (4.4) we have added extra spaces writing down the elements of the sets $\mathbb{N}_{1,2}, \mathbb{N}_{2,2}, \mathbb{N}_{1,3}, \mathbb{N}_{2,3}, \mathbb{N}_{3,3}$ just to emphasize Postulate 3 and to show visually that $\mathbb{N}_{1,2} \cup \mathbb{N}_{2,2}=\mathbb{N}$ and $\mathbb{N}_{1,3} \cup \mathbb{N}_{2,3} \cup \mathbb{N}_{3,3}=\mathbb{N}$.

In general, to introduce $\frac{1}{n}$ we do not try to count elements $k, k+n, k+2 n, k+3 n, \ldots$ one by one in (4.2). In fact, we cannot do this due to Postulate 1. By using Postulate 3, 
we construct the sets $\mathbb{N}_{k, n}, 1 \leq k \leq n$, by separating the whole, i.e., the set $\mathbb{N}$, in $n$ parts and we affirm that the number of elements of the $n$th part of the set, i.e., $\frac{1}{n}$, is $n$ times less than the number of elements of the entire set, i.e., than (1).

As was already mentioned, in terms of our granary example (1) can be interpreted as the number of seeds in the sack. In that example, the number $K_{1}$ of seeds in each sack was fixed and finite but it was impossible to express it in units $u_{0}$, i.e., seeds, by counting seed by seed because we had supposed that sacks were very big and the corresponding number would not be expressible by available numerals. In spite of the fact that $K_{1}, K_{2}$, and $K_{3}$ were inexpressible and unknown, by using new units of measure (sacks, trucks, etc.) it was possible to count more easily and to express the required quantities. Now our sack has the infinite but again fixed number of seeds. It is fixed because it has a strong link to a concrete set - it is the number of elements of the set of natural numbers. Since this number is inexpressible by existing numeral systems with the same accuracy afforded to measure finite small sets ${ }^{9}$, we introduce a new numeral, (1), to express the required quantity. Then, we apply Postulate 3 and say that if the sack contains (1) seeds, then, even though we are not able to count the number of seeds of the $n$th part of the sack seed by seed, its $n$th part contains $n$ times less seeds than the entire sack, i.e., $\frac{1}{n}$ seeds. Notice that the numbers $\frac{1}{n}$ are integer since they have been introduced as numbers of elements of sets $\mathbb{N}_{k, n}$.

The new unit of measure allows us to express a variety of infinite numbers (including those larger than (1) that will be considered later) and calculate easily the number of elements of the union, intersection, difference, or product of sets of type $\mathbb{N}_{k, n}$. Due to our accepted methodology, we do it in the same way as these measurements are executed for finite sets. Let us consider two simple examples showing how grossone can be used for this purpose (a general rule for determining the number of elements of infinite sets having a more complex structure will be given in Section 7).

Example 4.2. Let us determine the number of elements of the set $A_{k, n}=\mathbb{N}_{k, n} \backslash\{a\}$, $a \in \mathbb{N}_{k, n}, n \geq 1$. Due to the IUA, the set $\mathbb{N}_{k, n}$ has $\frac{1}{n}$ elements. The set $A_{k, n}$ has been constructed by excluding one element from $N_{k, n}$. Thus, the set $A_{k, n}$ has $\frac{1}{n}-1$ elements. The granary interpretation can be also given for the number $\frac{1}{n}-1$ : the number of seeds in the $n$th part of the sack minus one seed. For $n=1$ we have (1) -1 interpreted as the number of seeds in the sack minus one seed.

Divisibility and Example 4.2 show us that in addition to the usual way of counting, i.e., by adding units, that has been formalized in the traditional Mathematics, there exists also the way to count by taking parts of the whole and by subtracting units or parts of the whole. The following example shows a slightly more complex situation (other more

\footnotetext{
${ }^{9}$ First, this quantity is inexpressible by numerals used to count the number of elements of finite sets because the set $\mathbb{N}$ is infinite. Second, traditional numerals existing to express infinite numbers do not have the required high accuracy (recall that we would like to be able to register the variation of the number of elements of infinite sets even when one element has been excluded or added). For example, by using Cantor's alephs we say that cardinality of the sets $\mathbb{N}$ and $\mathbb{N} \backslash\{1\}$ is the same $-\aleph_{0}$. This answer is correct but its accuracy is low - we are not able to register the fact that one element was excluded from the set $\mathbb{N}$ even though we ourselves have executed this exclusion. Analogously, we can say that both the sets have many elements. Again, the answer 'many' is correct but its accuracy is low.
} 
sophisticated examples will be given later after the reader gets accustomed to the concept of (1).

Example 4.3. Let us consider the following two sets

$$
\begin{gathered}
B_{1}=\{4,9,14,19,24,29,34,39,44,49,54,59,64,69,74,79, \ldots\}, \\
B_{2}=\{3,14,25,36,47,58,69,80,91,102,113,124,135, \ldots\}
\end{gathered}
$$

and determine the number of elements in the set $B=\left(B_{1} \cap B_{2}\right) \cup\{3,4,5,69\}$. It follows immediately from the IUA that $B_{1}=\mathbb{N}_{4,5}$ and $B_{2}=\mathbb{N}_{3,11}$. Their intersection

$$
B_{1} \cap B_{2}=\mathbb{N}_{4,5} \cap \mathbb{N}_{3,11}=\{14,69,124, \ldots\}=\mathbb{N}_{14,55}
$$

and, therefore, due to the IUA, it has $\frac{1}{55}$ elements. Finally, since 69 belongs to the set $\mathbb{N}_{14,55}$ and 3,4, and 5 do not belong to it, the set $B$ has $\frac{1}{55}+3$ elements. The granary interpretation: this is the number of seeds in the 55th part of the sack plus three seeds.

Let us continue this presentation of properties of grossone by discussing one of the differences of the new approach with respect to non-standard analysis (see [82]). It consists of the fact that $(1) \in \mathbb{N}$ because grossone has been introduced as the quantity of natural numbers. Similarly, the number 5 being the number of elements of the set

$$
A=\{1,2,3,4,5\}
$$

is the largest element in this set. As was already mentioned, the numeral (1) allows one to write down the set, $\mathbb{N}$, of natural numbers in the form

$$
\mathbb{N}=\left\{1,2, \ldots \frac{1}{2}-2, \frac{1}{2}-1, \frac{1}{2}, \frac{1}{2}+1, \frac{1}{2}+2, \ldots \text { (1) }-2, \quad(1)-1, \quad(1)\right\} .
$$

and to observe infinite natural numbers

$$
\ldots \quad \frac{1}{2}-2, \frac{1}{2}-1, \frac{1}{2}, \frac{1}{2}+1, \frac{1}{2}+2, \ldots \text { (1) }-2,(1)-1,(1)
$$

that are invisible if traditional numeral systems are used to observe $\mathbb{N}$. Similarly, Pirahã are not able to see finite natural numbers larger than 2 .

This example illustrates also the fact that when we speak about sets (finite or infinite) it is necessary to take care about tools used to describe a set (recall Postulate 2). In order to introduce a set, it is necessary to have a language (e.g., a numeral system) allowing us to describe both its elements and the number of the elements in the set. For instance, the set $A$ from (4.5) cannot be defined using the mathematical language of Pirahã.

Analogously, the words 'the set of all finite numbers' do not define a set completely from our point of view. It is always necessary to specify which instruments are used to describe (and to observe) the required set and, as a consequence, to speak about 'the set of all finite numbers expressible in a fixed numeral system'. For instance, for Pirahã 'the set of all finite numbers' is the set $\{1,2\}$ and for Mundurukú 'the set of all finite numbers' is the set $A$ from (4.5). As it happens in Physics, the instrument used for an 
observation bounds the possibility of the observation. It is not possible to say how we shall see the object of our observation if we have not clarified which instruments will be used to execute the observation (see discussion on page 15). Thus, in the moment one chooses the instrument of the observation this person chooses also objects that can be observed.

In order to be more acquainted with this methodology separating objects and tools of their observation let us try to answer to the following question: Which natural numbers can we express by using the numeral (1)? Analogously to the examples with the phrases 'the set of all finite numbers' and the question 'What do you see in this direction?' this question is not sufficiently precise since it does not specify which numeral system is used to express finite quantities. Suppose that we have fixed a numeral system, $\mathcal{S}$, for expressing finite natural numbers and it allows us to express $K_{\mathcal{S}}$ numbers (not necessarily consecutive) belonging to a set $\mathcal{N}_{S} \subset \mathbb{N}$. Note that due to Postulate $1, K_{\mathcal{S}}$ is finite. Then, addition of (1) to this numeral system will allow us to express also infinite natural numbers $\frac{i(1)}{n} \pm k \leq 1$ where $1 \leq i \leq n, k \in \mathcal{N}_{S}, n \in \mathcal{N}_{S}$ (notice that since $\frac{1}{n}$ are integers, $\frac{i(1)}{n}$ are integers too). Thus, the more powerful system $\mathcal{S}$ is used to express finite numbers, the more infinite numbers can be expressed but their quantity is always finite, again due to Postulate 1. The (1)-based numeral system allows us to express more numbers than traditional numeral systems thanks to the introduced new numerals, however, as it happens for all numeral systems, its abilities to express numbers are also limited.

Example 4.4. Let us consider the numeral system, $\mathcal{P}$, of Pirahã able to express only numbers 1 and 2 (the only difference will be in the usage of numerals ' 1 ' and ' 2 ' instead of original numerals $I$ and $I I$ used by Pirahã). If we add to $P$ the new numeral (1), we obtain a new numeral system (we call it $\widehat{P}$ ) allowing us to express only ten numbers represented by the following numerals

$$
\underbrace{1,2}_{\text {finite }}, \quad \cdots \quad \underbrace{\frac{1}{2}-2, \frac{1}{2}-1, \frac{1}{2}, \frac{1}{2}+1, \frac{1}{2}+2}_{\text {infinite }}, \quad \cdots \quad \underbrace{(1)-2,(1)-1,1}_{\text {infinite }} .
$$

The first two numbers in (4.8) are finite, the remaining eight are infinite. Traditionally dots in a record of the type $1,2,3, \ldots$ show that we do not want but can proceed with writing. Dots in (4.8) have a different meaning: they represent natural numbers that are not expressible in the numeral system $\widehat{P}$.

As a consequence, $\widehat{P}$ does not allow us to execute such operation as $2+2$ or to add 2 to $\frac{1}{2}+2$ because their results cannot be expressed in $\widehat{\mathcal{P}}$. Of course, we do not say that results of these operations are equal to 'many' (as Pirahã do for operations $2+2$ and $2+1$ ). We just say that the results are not expressible in $\widehat{\mathcal{P}}$ and it is necessary to take another, more powerful numeral system if we want to execute these operations. This represents an important shift in the methodology applied in situations where one faces a difficulty in counting. Instead of concluding that the problem is hard one says that the used instrument is inadequate and, thefore, should be substituted with a more powerful tool.

Note that crucial limitations discussed in Example 4.4 hold for sets, too. In fact, the numeral system $\mathcal{P}$ allows us to define only the sets $\mathbb{N}_{1,2}$ and $\mathbb{N}_{2,2}$ among all possible sets of 
the form $\mathbb{N}_{k, n}$ from (4.2) because we have only two finite numerals, ' 1 ' and ' 2 ', in $\mathcal{P}$. This numeral system is too weak to define other sets of this type, for instance, $\mathbb{N}_{4,5}$, because numbers greater than 2 required for these definition are not expressible in $\mathcal{P}$. Moreover in each of the sets $\mathbb{N}_{1,2}$ and $\mathbb{N}_{2,2}$ the system $\mathcal{P}$ allows us to observe only one element ( 1 in $\mathbb{N}_{1,2}$ and 2 in $\mathbb{N}_{2,2}$ ). These limitations have a general character and are related to all questions requiring a numerical answer (i.e., an answer expressed only in numerals, without variables). In order to obtain such an answer, it is necessary to know at least one numeral system able to express numerals required to write down this answer.

We are now ready to state the following result being a direct consequence of the accepted methodological postulates.

Theorem 4.5. The set $\mathbb{N}$ is not a monoid under addition.

Proof. Due to Postulate 3, the operation (1) +1 gives us as the result a number greater than (1). Thus, by definition of grossone, $1+1$ does not belong to $\mathbb{N}$ and, therefore, $\mathbb{N}$ is not closed under addition and is not a monoid.

This result also means that adding the IUA to the axioms of natural numbers allows us to talk about the set of extended natural numbers indicated as $\widehat{\mathbb{N}}$ and including $\mathbb{N}$ as a proper subset

$$
\begin{aligned}
& \widehat{\mathbb{N}}=\{\underbrace{1,2, \ldots,(1)-1,(1)}_{\text {Natural numbers }},(1)+1,(1)+2, \ldots, 2(1)-1,2(1), 2(1)+1, \ldots \\
& \left.(1)^{2}-1,(1)^{2},(1)^{2}+1, \ldots 3(1)^{\Phi}-1,3(1)^{\Phi}, 3(1)^{\oplus}+1, \ldots\right\} .
\end{aligned}
$$

The extended natural numbers greater than grossone are also linked to sets of numbers and can be interpreted in the terms of grain.

Example 4.6. Let us determine the number of elements of the set

$$
C_{m}=\left\{\left(a_{1}, a_{2}, \ldots, a_{m-1}, a_{m}\right): a_{i} \in \mathbb{N}, 1 \leq i \leq m\right\}, \quad 2 \leq m \leq 1 .
$$

The elements of $C_{m}$ are $m$-tuples of natural numbers. It is known from combinatorial calculus that if we have $m$ positions and each of them can be filled in by one of $l$ symbols, the number of the obtained $m$-tuples is equal to $l^{m}$. In our case, since $\mathbb{N}$ has grossone elements, $l=(1)$. Thus, the set $C_{m}$ has $\left(1^{m}\right.$ elements. In the particular case, $m=2$, we obtain that the set

$$
C_{2}=\left\{\left(a_{1}, a_{2}\right): a_{i} \in \mathbb{N}, i \in\{1,2\}\right\},
$$

being the set of couples of natural numbers, has $\left(1^{2}\right.$ elements. This fact is illustrated below
$(1,1)$
$(1,2)$
$\ldots$
$(1,1-1)$,
(1, (1),
$(2,1)$
$(2,2)$
$\ldots$
$(2,1-1)$,
(2, (1),
(1) $-1,1)$,
(1) $-1,2)$
..
(1), 1),
(1), 2),
(1) $-1,1,-1)$,
(1) -1, , 1),
(1), (1)-1),
(1), (1). 
Another interesting particular case is the set

$$
C_{\mathbb{1}}=\left\{\left(a_{1}, a_{2}, \ldots, a_{\mathbb{1}-1}, a_{\circledast}\right): a_{i} \in \mathbb{N}, 1 \leq i \leq \mathbb{1}\right\}
$$

having (1) ${ }^{\mathbb{1}}$ elements.

Note that we can also give the granary interpretation for the numbers of the type $\mathbb{1}^{m}$ : if we accept that the numbers $K_{i}$ from page 17 are such that $K_{i}=1,1 \leq i \leq m-1$, then (1) ${ }^{2}$ can be viewed as the number of seeds in the truck, $1^{3}$ as the number of seeds in the train waggon, etc.

Example 4.7. Another important set having more than grossone elements is the set, $\mathbb{Z}$, of integers. Clearly, it has 2(1)+1 elements (1) positive numbers, (1) negative, and zero).

The set, $\widehat{\mathbb{Z}}$, of extended integer numbers can be constructed from the set, $\mathbb{Z}$, of integers by a complete analogy and inverse elements with respect to addition are introduced naturally. For example, 7(1) has its inverse with respect to addition equal to -71.

Let us emphasize that, due to Postulates 1 and 2, the new system of counting cannot give answers to all questions regarding infinite sets. What can we say, for instance, about the number of elements of the sets $\widehat{\mathbb{N}}$ and $\widehat{\mathbb{Z}}$ ? Have they been described completely? The introduced numeral system based on (1) is too weak to give answers to these questions since it does not allow us to express the number of elements of these sets. It is necessary to introduce in a way a more powerful numeral system by defining new numerals (for instance, (2), (3), etc).

We conclude this subsection by the following remark. The IUA describes a new number - the quantity of elements in the set of natural numbers - expressed by the numeral (1). However, other numerals and sets can be used to express the idea of the axiom. For example, the numeral 1 can be introduced as the number of elements of the set, $\mathbb{E}$, of even numbers and can be taken as the base of a numeral system. In this case, the IUA can be reformulated using the numeral 1 and numerals including it will be used to express infinite numbers. For example, the numbers of elements $|\mathbb{O}|$ of the set, $\mathbb{O}$, of odd numbers and numbers of elements of the sets $\mathbb{N}$ and $\mathbb{Z}$ will be expressed as

$$
|\mathbb{O}|=|\mathbb{E}|=\mathbf{1}, \quad|\mathbb{N}|=2 \cdot \boldsymbol{1}, \quad|\mathbb{Z}|=4 \cdot \mathbf{1}+1 .
$$

We emphasize through this remark that infinite numbers (similarly to the finite ones) can be expressed by various numerals and in different numeral systems.

4.2. Postulate 3 versus bijections of infinite sets: no contradiction. Let us consider how the principle 'The part is less than the whole' included in Postulate 3 and the IUA can be reconciled with traditional views on infinite sets. At first sight it seems that there is a contradiction between the two positions. For instance, traditionally it is said that the oneto-one correspondence can be established between the sets of natural and odd numbers. Namely, odd numbers can be put in a one-to-one correspondence with all natural numbers in spite of the fact that $\mathbb{O}$ is a proper subset of $\mathbb{N}$

$$
\begin{array}{lccccccc}
\text { odd numbers: } & 1, & 3, & 5, & 7, & 9, & 11, & \ldots \\
& \uparrow & \uparrow & \uparrow & \uparrow & \uparrow & \uparrow & \\
\text { natural numbers: } & 1, & 2, & 3, & 4 & 5, & 6, & \ldots
\end{array}
$$


The traditional conclusion from (4.10) is that both sets are countable and they have the same cardinality $\boldsymbol{\aleph}_{0}$.

Let us see now what we can say from the new methodological position, in particular, by using Postulate 2. The separation of the objects of study which are two infinite sets from the instrument used to compare them, i.e., from the bijection, suggests that another conclusion can be derived from (4.10): the accuracy of the used instrument is not sufficiently high to see the difference between the sizes of the two sets.

We have already seen that when one executes the operation of counting, the accuracy of the result depends on the numeral system used for counting. If one asked Pirahã to measure sets consisting of four apples and five apples the answer would be that both sets of apples have many elements. This answer is correct but its precision is low due to the weakness of the numeral system used to measure the sets.

Thus, the introduction of the notion of accuracy for measuring sets is very important and should be applied to infinite sets also. As was already discussed, since for cardinal numbers it follows

$$
\aleph_{0}+1=\aleph_{0}, \quad \aleph_{0}+2=\aleph_{0}, \quad \aleph_{0}+\aleph_{0}=\aleph_{0},
$$

these relations suggest that the accuracy of the cardinal numeral system of Alephs is not sufficiently high to see the difference with respect to the number of elements of the two sets from (4.10).

In order to look at the record (4.10) using the new methodology let us remind that due to the IUA the sets of even and odd numbers have (1)/2 elements each and, therefore, (1) is even. It is also necessary to recall that numbers that are larger than (1) are not natural, they are extended natural numbers. For instance, (1) +1 is odd but not natural, it is the extended natural, see (4.9). Thus, the last odd natural number is (1)-1. Since the number of elements of the set of odd numbers is equal to $\frac{1}{2}$, we can write down not only the initial (as it is usually done traditionally) but also the final part of (4.10)

$$
\begin{array}{cccccccccc}
1, & 3, & 5, & 7, & 9, & 11, & \ldots & 11-5, & 11-3, & 1-1 \\
\uparrow & \uparrow & \uparrow & \uparrow & \uparrow & \uparrow & & \downarrow & \uparrow & \uparrow \\
1, & 2, & 3, & 4 & 5, & 6, & \ldots & \frac{1}{2}-2, & \frac{1}{2}-1, & \frac{1}{2}
\end{array}
$$

concluding so (4.10) in a complete accordance with the principle 'The part is less than the whole'. Both records, (4.10) and (4.11), are correct but (4.11) is more accurate, since it allows us to observe the final part of the correspondence that is invisible if (4.10) is used.

The accuracy of the (1)-based numeral system allows us to measure also, for instance, such sets as $\mathbb{O}^{\prime}=\mathbb{O} \backslash\{3\}$ and $\mathbb{O}^{\prime \prime}=\mathbb{O} \backslash\{1,1-1\}$. The set $\mathbb{O}^{\prime}$ is constructed by excluding one element from $\mathbb{O}$ and the set $\mathbb{O}^{\prime \prime}$ by excluding from $\mathbb{O}$ two elements. Thus, $\mathbb{O}^{\prime}$ and $\mathbb{O}^{\prime \prime}$ have $\frac{1}{2}-1$ and $\frac{1}{2}-2$ elements, respectively. In case one wishes to establish the corresponding bijections, starting with natural numbers $1,2,3, \ldots$ we obtain for these two sets

$$
\begin{array}{cccccccccc}
1, & 5, & 7, & 9, & 11, & 13, & \ldots & 11-5, & 1-3, & 11-1 \\
\uparrow & \uparrow & \uparrow & \uparrow & \downarrow & \downarrow & & \uparrow & \uparrow & \downarrow \\
1, & 2, & 3, & 4 & 5, & 6, & \ldots & \frac{1}{2}-3, & \frac{1}{2}-2, & \frac{1}{2}-1
\end{array}
$$




$$
\begin{aligned}
& 3, \quad 5, \quad 7, \quad 9, \quad 11, \quad 13, \ldots . \quad 11-7, \quad 1-5, \quad 1-3
\end{aligned}
$$

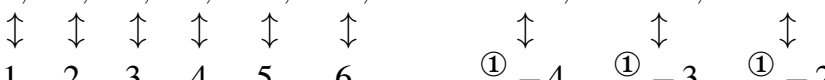

In order to become more familiar with natural and extended natural numbers and bijections let us consider one more example.

Example 4.8. Suppose that we wish to multiply each element of the set of natural numbers, $\mathbb{N}$, by 2 . We would like to study the resulting set, that is called $\mathbb{E}^{2}$ hereinafter, to calculate the number of its elements, and to specify which among its elements are natural and which ones are extended natural numbers and how many they are.

The introduction of the (1)-based numeral system allows us to write down the sets of natural and extended natural numbers in the form (4.9). By definition, the number of elements of $\mathbb{N}$ is equal to (1). Thus, after multiplication of each of the elements of $\mathbb{N}$ by 2 , the resulting set, $\mathbb{E}^{2}$, will also have grossone elements. In particular, the number $\frac{1}{2}$ multiplied by 2 gives us (1) and $\frac{1}{2}+1$ multiplied by 2 gives us 1 that is even extended natural number, see (4.9). Analogously, the last element of $\mathbb{N}$, i.e., (1), multiplied by 2 gives us 21. Thus, the set $\mathbb{E}^{2}$ can be written as follows

$$
\mathbb{E}^{2}=\{2,4,6, \ldots \text { (1) }-2,(1),(1)+2, \ldots \text { 2(1) }-4,2(1)-2,2(1)\}
$$

and the corresponding bijection is

$$
\begin{aligned}
& 2, \quad 4, \quad 6, \quad \ldots \quad(1)-2, \quad(1), \quad(1)+2, \quad \ldots \quad 21-4, \quad 2(1)-2, \quad 2(1)
\end{aligned}
$$

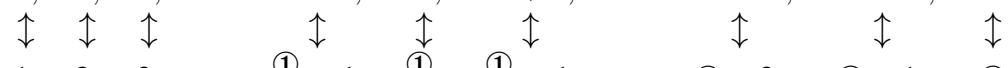

$$
\begin{aligned}
& 1, \quad 2, \quad 3, \quad \ldots \quad \frac{1}{2}-1, \quad \frac{1}{2}, \quad \frac{1}{2}+1, \ldots \quad(1-2, \quad 11-1, \quad \text { (1) }
\end{aligned}
$$

where numbers $\{2,4,6, \ldots$ (1) $-4,1)-2,1\}$ are even and natural (they are $\frac{1}{2}$ ) and numbers $\{(1)+2,1)+4, \ldots 2(1)-4,21-2,21\}$ are even and extended natural, they also are $\frac{1}{2}$.

The expressed viewpoint on infinite sets allows us to avoid a number of paradoxes related traditionally to infinite sets. Let us illustrate this fact by considering Hilbert's paradox of the Grand Hotel. In a hotel having a finite number of rooms no more new guests can be accommodated if it is full. Hilbert's Grand Hotel has an infinite number of rooms (of course, the number of rooms is countable, because the rooms in the Hotel are numbered). If a new guest arrives at the Hotel where every room is occupied, it is, nevertheless, possible to find a room for the newcomer. To do so, it is necessary to move the guest occupying room 1 to room 2 , the guest occupying room 2 to room 3 , etc. In such a way room 1 will be ready for the new guest and, in spite of the assumption that there are no available rooms in the Hotel, an empty room is found.

Let us consider now the situation in the (1)-based framework. In the paradox, the number of rooms in the Hotel is infinite. In the new terminology it is not sufficient to say this, it is required to indicate explicitly the number of the rooms. Suppose that the Hotel has (1) rooms. When a new guest arrives, it is proposed to move the guest occupying 
room 1 to room 2 , the guest occupying room 2 to room 3 , etc. At the end of this procedure the guest from the last room number (1) should be moved to the room (1)+1. However, the Hotel has only (1) rooms and, therefore, the poor guy from the room (1) will go out of the Hotel (the situation taking place in hotels with a finite number of rooms if such a procedure is implemented).

Notice, that there is no contradiction between the two ways to see the situation. The traditional answer is that it is possible to put the newcomer in the first room. The (1)-way of doing confirms this result but shows a thing that was invisible traditionally - the guest from the last room should go out of the Hotel.

4.3. Positional numeral system with the infinite radix (1). We have already started to write down simple infinite numbers and to execute arithmetical operations with them without concentrating our attention upon this question. Let us consider it systematically.

Different numeral systems have been developed to describe finite numbers. In positional numeral systems, fractional numbers are expressed by the record

$$
\left(a_{n} a_{n-1} \ldots a_{1} a_{0} \cdot a_{-1} a_{-2} \ldots a_{-(q-1)} a_{-q}\right)_{b}
$$

where numerals $a_{i},-q \leq i \leq n$, are called digits, belong to the alphabet $\{0,1, \ldots, b-1\}$, and the dot is used to separate the fractional part from the integer one. Thus, the numeral (4.14) expresses the quantity obtained by summing up

$$
a_{n} b^{n}+a_{n-1} b^{n-1}+\ldots+a_{1} b^{1}+a_{0} b^{0}+a_{-1} b^{-1}+\ldots+a_{-(q-1)} b^{-(q-1)}+a_{-q} b^{-q} .
$$

Record (4.14) uses numerals consisting of one symbol each, i.e., digits $a_{i} \in\{0,1, \ldots, b-$ $1\}$, to express how many finite units of the type $b^{i}$ belong to the number (4.15). Quantities of finite units $b^{i}$ are counted separately for each exponent $i$ and all symbols in the alphabet $\{0,1, \ldots, b-1\}$ express finite numbers.

To express infinite and infinitesimal numbers we shall use records that are similar to (4.14) and (4.15) but have some peculiarities. In order to construct a number $C$ in the numeral positional system with the base (1), we subdivide $C$ into groups corresponding to powers of (1):

$$
C=c_{p_{m}} 1^{p_{m}}+\ldots+c_{p_{1}}{ }^{1}{ }^{p_{1}}+c_{p_{0}}{ }^{1}{ }^{p_{0}}+c_{p_{-1}}{ }^{1} p^{p_{-1}}+\ldots+c_{p_{-k}}{ }^{1} p^{p_{-k}}
$$

Then, the record

$$
C=c_{p_{m}}{ }^{(1)}{ }^{p_{m}} \ldots c_{p_{1}}{ }^{1}{ }^{p_{1}} c_{p_{0}}{ }^{(1)^{p_{0}}} c_{p_{-1}}{ }^{1} p^{p_{-1}} \ldots c_{p_{-k}}{ }^{1} p^{p_{-k}}
$$

represents the number $C$, where all numerals $c_{i}$ are called grossdigits. They are not equal to zero and belong to a traditional numeral system. Grossdigits express finite positive or negative numbers and show how many corresponding units (1) ${ }^{p_{i}}$ should be added or subtracted in order to form the number $C$. Grossdigits can be expressed by several symbols using positional systems, can be written in the form $\frac{Q}{q}$ where $Q$ and $q$ are integers, or in any other numeral system used to express finite quantities. 
Numbers $p_{i}$ in (4.17) called grosspowers can be finite, infinite, and infinitesimal (the introduction of infinitesimal numbers will be given soon), they are sorted in the decreasing order

$$
p_{m}>p_{m-1}>\ldots>p_{1}>p_{0}>p_{-1}>\ldots p_{-(k-1)}>p_{-k}
$$

with $p_{0}=0$.

In the traditional record (4.14), there exists a convention that a digit $a_{i}$ shows how many powers $b^{i}$ are present in the number and the radix $b$ is not written explicitly. In the record (4.17), we write (1) ${ }^{p_{i}}$ explicitly because in the (1)-based positional system the number $i$ in general is not equal to the grosspower $p_{i}$. This gives the possibility to write, for example, such a number as 7.6(1 ${ }^{244.5} 341^{32}$ having grosspowers $p_{2}=244.5, p_{1}=32$ and grossdigits $c_{244.5}=7.6, c_{32}=34$ without indicating grossdigits equal to zero corresponding to grosspowers smaller than 244.5 and greater than 32 . Note also that if a grossdigit $c_{p_{i}}=1$ then we often write (1) $^{p_{i}}$ instead of $11^{p_{i}}$.

The term having $p_{0}=0$ represents the finite part of $C$ because, due to (4.1), we have $c_{0}{ }^{0}{ }^{0}=c_{0}$. The terms having finite positive grosspowers represent the simplest infinite parts of $C$. Analogously, terms having negative finite grosspowers represent the simplest infinitesimal parts of $C$. For instance, the number $\left(^{-1}=\frac{1}{1}\right.$ is infinitesimal. It is the inverse element with respect to multiplication for (1):

$$
(1)^{-1} \cdot(1)=(1) \cdot(1)^{-1}=1 \text {. }
$$

Note that all infinitesimals are different from zero. Particularly, $\frac{1}{1}>0$ because it is a result of division of two positive numbers. It also has a clear granary interpretation. Namely, if we have a sack containing (1) seeds, then one sack divided by the number of seeds in it is equal to one seed. Vice versa, one seed, i.e., $\frac{1}{1}$, multiplied by the number of seeds in the sack, (1), gives one sack of seeds.

All of the numbers introduced above can be grosspowers, as well, giving so a possibility to have various combinations of quantities and to construct terms having a more complex structure ${ }^{10}$.

Example 4.9. The left-hand expression below shows how to write down numbers in the new numeral system and the right-hand shows how the value of the number is calculated:

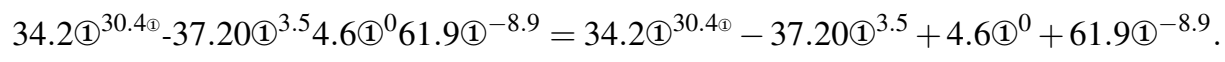

\footnotetext{
${ }^{10}$ At the first glance the record (4.16) (and, therefore, the numerals (4.17)) can remind one of numbers from the Levi-Civita field (see [64]) that is a very interesting and important precedent of algebraic manipulations with infinities and infinitesimals. However, the two mathematical objects have several crucial differences. They have been introduced for different purposes by using two mathematical languages having different accuracies and on the basis of different methodological foundations. In fact, Levi-Civita does not discuss the distinction between numbers and numerals and works with generic numbers whereas each numeral (4.17) represents a concrete number. Numbers of Levi-Civita have neither cardinal nor ordinal properties; they are built using a generic infinitesimal and only its rational powers are allowed; Levi-Civita uses symbol $\infty$ in his construction; there is no numeral system that would allow one to assign numerical values to his numbers; it is not explained how it would be possible to pass from a generic infinitesimal $h$ to a concrete one (see also the discussion above on the distinction between numbers and numerals).

This should not be taken as a criticism of Levi-Civita's results. The above discussion has been introduced in this text just to underline that we are in front of two different mathematical tools that should be used in different mathematical contexts.
} 


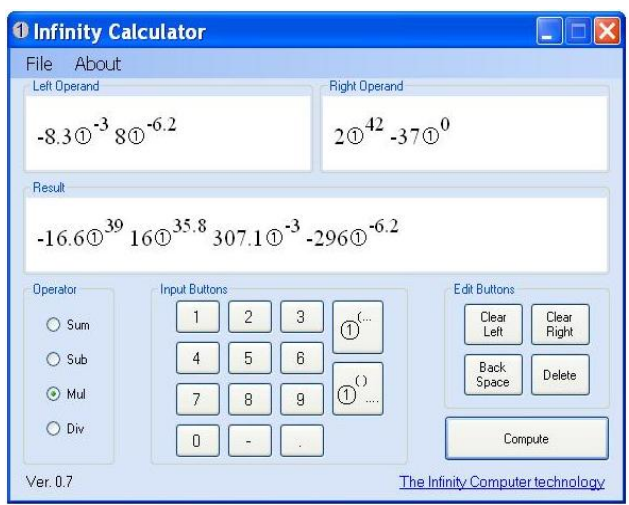

Figure 4.1. Operation of multiplication executed on the Infinity Calculator

The number above has one infinite part having an infinite grosspower, one infinite part having a finite grosspower, a finite part, and an infinitesimal part.

Finally, numbers having a finite and infinitesimal parts can be also expressed in the new numeral system, for instance, the number $-13.51^{0}-37\left(1^{-2} 111^{-16 \bowtie+4.3}\right.$ has a finite and two infinitesimal parts, the second of them has the infinite negative grosspower equal to $-16(1)+4.3$. In case a finite number has no infinitesimal parts it is called purely finite.

4.4. Arithmetical operations with (1)-based numerals. In this subsection, we describe arithmetical operations with the (1)-based positional numeral system. A working software simulator of the Infinity Computer has been implemented and the first application - the Infinity Calculator - has been realized. Fig. 4.1 shows operation of multiplication executed on the Infinity Calculator that works using the Infinity Computer technology. The left operand has two infinitesimal parts and the right operand has an infinite part and a finite one. The result has two infinite and two infinitesimal parts. Fig. 4.2 shows a division giving as the result an infinitesimal number consisting of three parts.

We start by discussing the operation of addition (subtraction is a direct consequence of addition and is thus omitted). Let us consider two numbers $A$ and $B$, where

$$
A=\sum_{i=1}^{K} a_{k_{i}}{ }^{1^{k_{i}}}, \quad B=\sum_{j=1}^{M} b_{m_{j}}(1)^{m_{j}}, \quad C=\sum_{i=1}^{L} c_{l_{i}}{ }^{1^{l_{i}}}
$$

and the result $C=A+B$ is constructed by including in it all items $a_{k_{i}}{ }^{\left(1 k^{k_{i}}\right.}$ from $A$ such that $k_{i} \neq m_{j}, 1 \leq j \leq M$, and all items $b_{m_{j}}{ }^{1^{m_{j}}}$ from $B$ such that $m_{j} \neq k_{i}, 1 \leq i \leq K$. If in $A$ and $B$ there are items such that $k_{i}=m_{j}$, for some $i$ and $j$, then this grosspower $k_{i}$ is included in $C$ with the grossdigit $b_{k_{i}}+a_{k_{i}}$, i.e., as $\left(b_{k_{i}}+a_{k_{i}}\right) 1^{k_{i}}$.

Example 4.10. We consider two infinite numbers $A$ and $B$, where

$$
A=16.5(1)^{44.2}(-12)(1)^{12} 17(1)^{0}, \quad B=6.23(1)^{3} 10.11^{0} 15(1)^{-4.1} .
$$




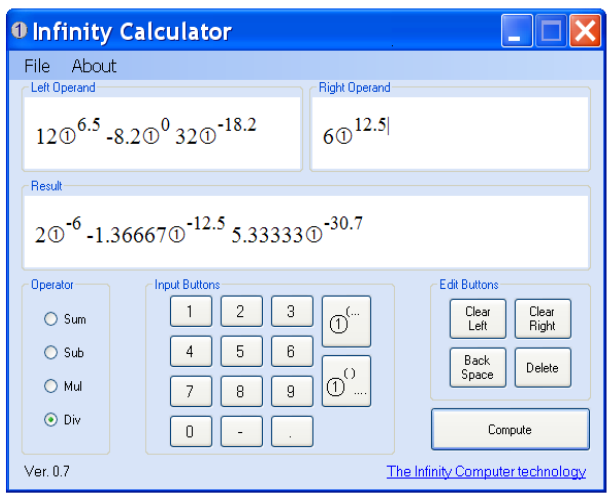

Figure 4.2. Operation of division executed on the Infinity Calculator

Their sum $C$ is calculated as follows:

$$
\begin{gathered}
\left.C=A+B=16.5(1)^{44.2}+(-12)(1)^{12}+171^{0}+6.23(1)^{3}+10.11\right)^{0}+15(1)^{-4.1}= \\
16.5(1)^{44.2}-12(1)^{12}+6.23(1)^{3}+27.111^{0}+15(1)^{-4.1}= \\
\left.\left.16.5(1)^{44.2}(-12)(1)^{12} 6.23(1)^{3} 27.11\right)^{0} 151\right)^{-4.1} .
\end{gathered}
$$

The operation of multiplication of two numbers $A$ and $B$ in the form (4.19) returns, as the result, the infinite number $C$ constructed as follows:

$$
C=\sum_{j=1}^{M} C_{j}, \quad C_{j}=b_{m_{j}}{ }^{1 m^{m_{j}}} \cdot A=\sum_{i=1}^{K} a_{k_{i}} b_{m_{j}}{ }^{(1)^{k_{i}+m_{j}}}, \quad 1 \leq j \leq M .
$$

Example 4.11. We consider two infinite numbers

$$
A=11^{18}(-5)(1)^{2.4}(-3)(1)^{1}, \quad B=-11^{1} 0.71^{-3}
$$

and calculate the product $C=B \cdot A$. The first partial product $C_{1}$ is equal to

$$
\begin{gathered}
\left.C_{1}=0.7(1)^{-3} \cdot A=0.71\right)^{-3}\left(1^{18}-5(1)^{2.4}-3(1)^{1}\right)= \\
\left.\left.0.71)^{15}-3.5(1)^{-0.6}-2.11\right)^{-2}=0.71\right)^{15}(-3.5)(1)^{-0.6}(-2.1)(1)^{-2} .
\end{gathered}
$$

The second partial product, $C_{2}$, is computed analogously

$$
\left.C_{2}=-(1)^{1} \cdot A=-1^{1}\left({ }^{1}\right)^{18}-51^{2.4}-3(1)^{1}\right)=-\left(1^{19} 51^{3.4} 3(1)^{2} .\right.
$$

Finally, the product $C$ is equal to

$$
C=C_{1}+C_{2}=-11^{19} 0.7(1)^{15} 5(1)^{3.4} 3(1)^{2}(-3.5)(1)^{-0.6}(-2.1)(1)^{-2} .
$$


In the operation of division of a number $C$ by a number $B$ from (4.19), we obtain a result $A$ and a reminder $R$ (that may equal zero), i.e., $C=A \cdot B+R$. The number $A$ is constructed as follows. The first grossdigit $a_{k_{K}}$ and the corresponding maximal exponent $k_{K}$ are established from the equalities

$$
a_{k_{K}}=c_{l_{L}} / b_{m_{M}}, \quad k_{K}=l_{L}-m_{M} .
$$

Then the first partial reminder $R_{1}$ is calculated as

$$
R_{1}=C-a_{k_{K}} 1^{k_{K}} \cdot B .
$$

If $R_{1} \neq 0$ then the number $C$ is substituted by $R_{1}$ and the process is repeated with a complete analogy. The grossdigit $a_{k_{K-i}}$, the corresponding grosspower $k_{K-i}$ and the partial reminder $R_{i+1}$ are computed by formulae (4.23) and (4.24) obtained from (4.21) and (4.22) as follows: $l_{L}$ and $c_{l_{L}}$ are substituted by the highest grosspower $n_{i}$ and the corresponding grossdigit $r_{n_{i}}$ of the partial reminder $R_{i}$ that, in turn, substitutes $C$ :

$$
\begin{gathered}
a_{k_{K-i}}=r_{n_{i}} / b_{m_{M}}, \quad k_{K-i}=n_{i}-m_{M} . \\
R_{i+1}=R_{i}-a_{k_{K-i}} 1^{k_{K-i}} \cdot B, \quad i \geq 1 .
\end{gathered}
$$

The process stops when a partial reminder equal to zero is found (this means that the final reminder $R=0$ ) or when a required accuracy of the result is reached.

Example 4.12. Let us divide the number $C=-101^{3} 16(1)^{0} 42(1)^{-3}$ by the number $B=$ $51^{3} 7$. For these numbers we have

$$
l_{L}=3, m_{M}=3, \quad c_{L}=-10, \quad b_{m_{M}}=5 .
$$

It follows immediately from (4.21) that $a_{k_{K}}\left(1^{k_{K}}=-21^{0}\right.$. The first partial reminder $R_{1}$ is calculated as

$$
\begin{gathered}
R_{1}=-10(1)^{3} 16(1)^{0} 42(1)^{-3}-\left(-2(1)^{0}\right) \cdot 5(1)^{3} 7= \\
-10(1)^{3} 16(1)^{0} 42(1)^{-3}+10(1)^{3} 14(1)^{0}=30(1)^{0} 42(1)^{-3} .
\end{gathered}
$$

By a complete analogy we should construct $a_{k_{K-1}} 1^{k_{K-1}}$ by rewriting (4.21) for $R_{1}$. By doing so we obtain equalities

$$
30=a_{k_{K-1}} \cdot 5, \quad 0=k_{K-1}+3
$$

and, as the result, $a_{k_{K-1}}$ (1) $^{k_{K-1}}=6$ (1) $^{-3}$. The second partial reminder is

$$
R_{2}=R_{1}-6(1)^{-3} \cdot 51^{3} 7=30(1)^{0} 42(1)^{-3}-30(1)^{0} 42(1)^{-3}=0 .
$$

Thus, we can conclude that the reminder $R=R_{2}=0$ and the final result of division is $A=-21^{0} 6(1)^{-3}$.

Let us now substitute the grossdigit 42 by 40 in $C$ and divide this new number $\widetilde{C}=$ $-10\left(1^{3} 16(1)^{0} 401^{-3}\right.$ by the same number $B=51^{3} 7$. This operation gives us the same result $\widetilde{A}_{2}=A=-21^{0} 6(1)^{-3}$ (where subscript 2 indicates that two partial reminders have been obtained) but with the reminder $\widetilde{R}=\widetilde{R}_{2}=-21^{-3}$. Thus, we obtain $\widetilde{C}=B \cdot \widetilde{A}_{2}+\widetilde{R}_{2}$. If we want to continue the procedure of division, we obtain $\left.\widetilde{A}_{3}=-21^{0} 6{ }^{-3}\right)^{-3}(-0.4)(1)^{-6}$ with the reminder $\widetilde{R}_{3}=0.281^{-6}$. Naturally, it follows $\widetilde{C}=B \cdot \widetilde{A}_{3}+\widetilde{R}_{3}$. The process continues until a partial reminder $\widetilde{R}_{i}=0$ is found or when a required accuracy of the result will be reached. 


\section{Numerical infinities and infinitesimals in applications}

The computational capabilities of the Infinity Computer allow one to construct new powerful numerical methods able to work with the infinities and infinitesimals introduced above. On several occasions it is possible to automatize the solution process of computational problems avoiding an interruption of the work of computer procedures and the necessity of a human intervention required when one works with traditional computers. It is worthwhile to stress again that examples described in this section are related to numerical computations on the Infinity Computer and symbolic computations are not used.

5.1. Higher order numerical differentiation on the Infinity Computer. In many practical applications it is necessary to calculate derivatives of a function $g(x)$ which is given by a computer procedure calculating its approximation $f(x)$. Since procedures for evaluating the exact values of $f^{\prime}(x)$ and higher derivatives are usually not available, either some numerical approximations are used for this purpose or automatic differentiation techniques are applied (see, e.g., [7, 9, 21, 26] and references given therein for a detailed discussion). Since the latter approaches usually allow one to evaluate $f^{\prime}(x)$ only, they are often symbolic and/or use specially developed procedures that re-write the code of $f(x)$ in order to obtain the code for $f^{\prime}(x)$, they are not considered in this text dedicated to numerical computations.

The simplest formulae used on traditional computers to approximate $f^{\prime}(x)$ require the evaluation of $f(x)$ at two points and use forward and backward differences

$$
f^{\prime}(x) \approx \frac{f(x+h)-f(x)}{h}, \quad f^{\prime}(x) \approx \frac{f(x)-f(x-h)}{h} .
$$

However, due to the finiteness of digits in the mantissa of floating-point numbers, roundoff errors in these procedures dominate calculation when $h \rightarrow 0$. Both $f(x+h)$ and $f(x-h)$ tend to $f(x)$, so that their difference tends to the difference of two almost equal quantities and thus contains fewer and fewer significant digits that provokes an explosion of the computational error. As an example, let us consider a computer procedure $f(x)$ implementing the function $g(x)=\frac{x+1}{x-1}$ and study errors provided by formulae (5.1) during approximation of the value $f^{\prime}(y)$ at the point $y=3$ in dependence of the step $h$. It can be seen from Fig. 5.1 that when $h$ becomes sufficiently small the error of approximation increases drastically.

Suppose now that we are in the (1)-based framework and a set of elementary functions $\left(\sin (x), \cos (x), a^{x}\right.$ etc.) is represented on the Infinity Computer by one of the usual ways used in traditional computers (see, e.g., [76]) involving the argument $x$, finite constants, and four arithmetical operations. A programmer writes a program $P$ that should calculate $g(x)$ using the said implementations of elementary functions, the argument $x$, and finite constants connected by four arithmetical operations. Obviously, $P$ calculates a numerical approximation $f(x)$ of the function $g(x)$. We suppose that $f(x)$ approximates $g(x)$ sufficiently well with respect to some criteria and we shall not discuss the goodness of this approximation here.

Then, as often happens in scientific computing, another person (a user) takes the program $P$ calculating $f(x)$ and is interested to calculate $f^{\prime}(x)$ and higher derivatives numeri- 


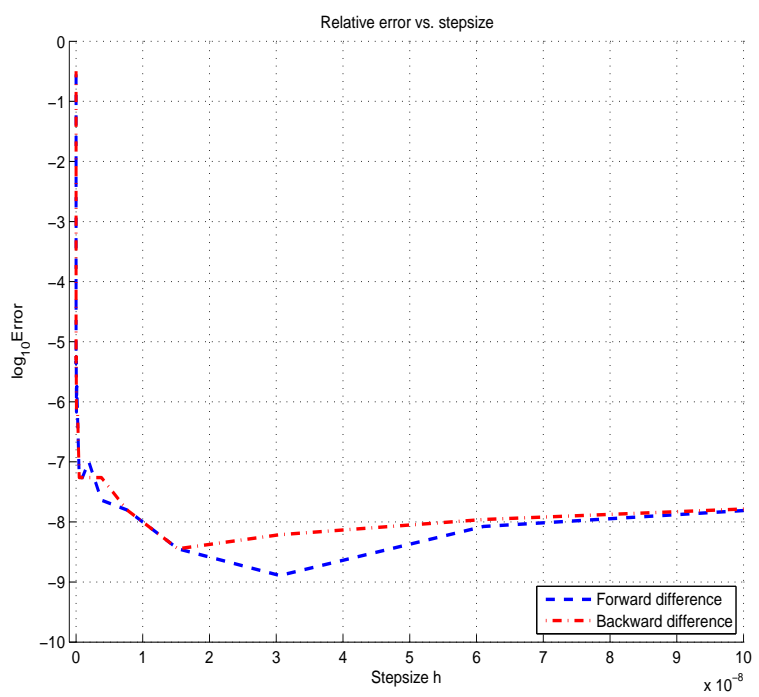

Figure 5.1. When $h$ becomes sufficiently small the error of approximation increases drastically

cally by using $P$. Suppose that computer programs for calculating $f^{\prime}(x), f^{\prime \prime}(x), \ldots f^{(k)}(x)$ and their analytical formulae are unavailable and the internal structure of the program calculating $f(x)$ is unknown to the user, i.e., it is a black-box.

In this situation, our attention will be attracted to the problem of a numerical calculation of the derivatives $f^{\prime}(x), f^{\prime \prime}(x), \ldots f^{(k)}(x)$ and to the information that can be obtained from the computer procedure $P$ calculating $f(x)$ for this purpose when it is executed on the Infinity Computer. The following theorem holds (for its proof and a detailed discussion on this topic see [95]).

Theorem 5.1. Suppose that: (i) for a function $f(x)$ calculated by a procedure implemented at the Infinity Computer there exists an unknown Taylor expansion in a finite neighborhood $\delta(y)$ of a purely finite point $y$; (ii) $f(x), f^{\prime}(x), f^{\prime \prime}(x), \ldots f^{(k)}(x)$ assume purely finite values or are equal to zero for purely finite points $x \in \delta(y)$; (iii) $f(x)$ has been evaluated at a point $y+1^{-1} \in \delta(y)$. Then the Infinity Computer returns the result of this evaluation in the positional numeral system with the infinite radix (1) in the following form

$$
\left.\left.f\left(y+(1)^{-1}\right)=c_{0} 1^{0} c_{-1} 1^{-1} c_{-2}{ }^{1}\right)^{-2} \ldots c_{-(k-1)}{ }^{1}{ }^{-(k-1)} c_{-k}{ }^{1}\right)^{-k},
$$

where

$$
f(y)=c_{0}, f^{\prime}(y)=c_{-1}, f^{\prime \prime}(y)=2 ! \cdot c_{-2}, \ldots f^{(k)}(y)=k ! \cdot c_{-k} .
$$

In order to illustrate the theorem let us consider the following example.

Example 5.2. Suppose that we have a computer procedure $f(x)$ implementing the already mentioned function $g(x)=\frac{x+1}{x-1}$ on the Infinity Computer and we want to evaluate the values $f(y), f^{\prime}(y), f^{\prime \prime}(y)$, and $f^{(3)}(y)$ at the point $y=3$. Suppose also that we do not know 
the analytical representation of $g(x)$ and $f(x)$ is given as a black-box, i.e., we supply an $x$ to the Infinity Computer and get $f(x)$ without knowing how this result has been computed.

Instead of using formulae (5.1) that, as has been discussed above, can lead to errors, it is proposed just to calculate $f(x)$ at the point $x=3+(1)^{-1}$. The result provided by the Infinity Computer is the following

$$
\left.\left.\left.f\left(3+1^{-1}\right)=21^{0}-0.511^{-1}+0.251\right)^{-2}-0.1251\right)^{-3}+0.06251\right)^{-4}-\ldots
$$

i.e., the result is a finite number with several infinitesimal parts (their number can be fixed a priori in dependence on the number of derivatives one wishes to calculate). From this numeral, by applying the theorem (see (5.3)) we obtain

$$
\begin{gathered}
f(3)=c_{0}=2, \quad f^{\prime}(3)=c_{-1}=-0.5, \quad f^{\prime \prime}(3)=2 ! \cdot c_{-2}=2 ! \cdot 0.25=0.5, \\
f^{(3)}(3)=3 ! \cdot c_{-3}=3 ! \cdot(-0.125)=0.75
\end{gathered}
$$

being exact values of $f(x)$ and of the respective derivatives at the point $y=3$. Thus, only one evaluation of $f(x)$ on the Infinity Computer gives us exact (recall that the word 'exact' means here the accuracy of the implementation of $f(x)$ ) derivatives whereas on a traditional computer it is necessary to evaluate $f(x)$ at least 4 times to get approximations of these values.

5.2. Infinitesimal perturbations for solving systems of linear equations. Very often in computations, an algorithm performing calculations of $f(x)$ at a point $x=a$ can encounter a situation where division by zero occurs. Then, obviously, this operation cannot be executed. If it is known that the problem under consideration has a finite solution $f(a)$, then a number of additional computational steps trying to avoid this division is performed. For instance, the computation is repeated several times at points $x=a-\varepsilon$ and $x=a+\varepsilon$ with different values of $\varepsilon$ and trying to understand where the sequences of values $f(a-\varepsilon)$ and $f(a+\varepsilon)$ converge. Notice that in the numbers $f(a-\varepsilon)$ and $f(a+\varepsilon)$ the contribution of $\varepsilon$ in the result cannot be separated from the contribution of $a$. For instance, suppose that we have $x=2, \varepsilon=0.001$, and after evaluating $f(2+0.001)$ we have got $f(2.001)=4.46$. This number, 4.46 , does not show us where there are the contribution of $x=2$ we are interested in and the contribution of the perturbation $\varepsilon=0.001$. These contributions are merged in the result 4.46 and cannot be separated. Thus, by choosing different values of $\varepsilon$ and by repeating computations many times it is possible to get only an approximation of the value $f(a)$.

On the other hand, we know that the Infinity Computer works by collecting different powers of (1) separately. Thus, by taking $\varepsilon=(1)^{-1}$ there is the possibility in certain cases to obtain the exact finite result $f(a)$ separated from the response provoked by the infinitesimal perturbation $\varepsilon=(1)^{-1}$.

A nice example of this kind is solving linear systems of equations. Traditionally, the operation of pivoting is used when one solves systems of linear equations by an algorithm such as, e.g., Gauss-Jordan elimination. Pivoting is the interchanging of rows (or both rows and columns) in order to avoid division by zero and to place a particularly 'good' element in the diagonal position prior to a particular operation. 
The following two illustrations give an idea of a numerical application of infinitesimal perturbations and show how their usage allows us to avoid pivoting in cases where the pivotal element is equal to zero. We emphasize again that the Infinity Computer (see [92]) works with infinities and infinitesimals expressed in the positional numeral system (4.16), (4.17) numerically, not symbolically.

Example 5.3. Let us consider the following simple linear system

$$
\left[\begin{array}{ll}
0 & 1 \\
2 & 2
\end{array}\right] \quad\left[\begin{array}{l}
x_{1} \\
x_{2}
\end{array}\right]=\left[\begin{array}{l}
2 \\
2
\end{array}\right]
$$

In case one decides to solve (5.4) by the method of Gauss-Jordan, the operation of pivoting should be performed because the first pivotal element $a_{11}=0$. Suppose now that we would like to avoid pivoting and, in order to illustrate the discussion on perturbations given above, introduce a finite perturbation $\varepsilon=0.01$ in the element $a_{11}=0$, i.e., put $a_{11}=0.01$. This gives us the possibility to perform Gauss transformations working with the perturbed system

$$
\left[\begin{array}{cc|c}
0.01 & 1 & 2 \\
2 & 2 & 2
\end{array}\right] \rightarrow\left[\begin{array}{cc|c}
1 & 100 & 200 \\
0 & -198 & -398
\end{array}\right] \rightarrow\left[\begin{array}{cc|c}
1 & 100 & 200 \\
0 & 1 & \frac{398}{198}
\end{array}\right] \rightarrow\left[\begin{array}{cc|c}
1 & 0 & \frac{-200}{198} \\
0 & 1 & \frac{398}{198}
\end{array}\right]
$$

and to obtain solution of the perturbed system $\tilde{x}_{1}^{*}=\frac{-200}{198}, \tilde{x}_{2}^{*}=\frac{398}{198}$ that, clearly, is not the solution to the original system (5.4).

Let us move now to (1)-based numerals, substitute the element $a_{11}=0$ by ${ }^{1}{ }^{-1}$, and perform Gauss-Jordan transformations without pivoting

$$
\begin{gathered}
{\left[\begin{array}{cc|c}
1)^{-1} & 1 & 2 \\
2 & 2 & 2
\end{array}\right] \rightarrow\left[\begin{array}{cc|c}
1 & 1 & 21 \\
0 & -2(1)+2 & -4(1)+2
\end{array}\right] \rightarrow\left[\begin{array}{cc|c}
1 & 1 & 2(1) \\
0 & 1 & \frac{-4(1)+2}{-2(1)+2}
\end{array}\right] \rightarrow} \\
{\left[\begin{array}{cc|c}
1 & 0 & 2(1)-\frac{-4(1)+2}{-2(1)+2} \\
0 & 1 & \frac{-4(1)+2}{-2(1)+2}
\end{array}\right] \rightarrow\left[\begin{array}{cc|c}
1 & 0 & \frac{2(1}{-2(1)+2} \\
0 & 1 & \frac{-4(1)+2}{-2(1)+2}
\end{array}\right] \rightarrow\left[\begin{array}{cc|c}
1 & 0 & -1+\frac{1}{1-(1)} \\
0 & 1 & 2-\frac{1}{1-(1)}
\end{array}\right] .}
\end{gathered}
$$

It follows immediately that, since the finite and infinitesimal parts can be separated on the Infinity Computer, the solution to the system (5.4) is given by the finite parts of numbers $-1+\frac{1}{1-(1)}$ and $2-\frac{1}{1-(1)}$, i.e., $x_{1}^{*}=-1$ and $x_{2}^{*}=2$.

In this example, we have introduced the number $\left(^{-1}\right.$ once and, as a result, we have obtained in the matrices expressions where the maximal power of grossone is one and there are rational expressions depending on grossone, as well. It is possible to manage these rational expressions in two ways: (i) to execute division in order to obtain its result in the form (4.16), (4.17); (ii) without executing division. In the latter case, we just continue to work with rational expressions. In the case (i), since we need finite numbers as final results, in the result of division it is not necessary to store the parts $c_{p}$ (1) $^{p}$ with $p<-1$. These parts can be forgotten because in any way the result of their successive multiplication with the numbers of the type $c_{1}{ }^{1}{ }^{1}$ (recall that 1 is the maximal exponent present in the matrix under consideration) will give exponents less than zero, i.e., numbers with 
these exponents will be infinitesimals that are not interesting for us in this computational context.

Thus, by using the positional numeral system (4.16), (4.17) with the radix grossone we obtain

$$
\begin{gathered}
{\left[\begin{array}{cc|c}
1 & 1 & 2(1) \\
0 & 1 & \frac{-4(1)+2}{-2(1)+2}
\end{array}\right] \rightarrow\left[\begin{array}{cc|c}
1 & 1 & 2(1) \\
0 & 1 & 21^{0}+1(1)^{-1}
\end{array}\right] \rightarrow} \\
{\left[\begin{array}{cc|c|c|c}
1 & 0 & 2(1)-\left(2(1)^{0} 1(1)^{-1}\right) \\
0 & 1 & 2(1)^{0} 11^{-1}
\end{array}\right] \rightarrow\left[\begin{array}{cc|c}
1 & 0 & -11^{0} \\
0 & 1 & 2(1)^{0} 11^{-1}
\end{array}\right] .}
\end{gathered}
$$

The finite parts of numbers $-11^{0}$ and $2(1)^{0} 11^{-1}$ then provide the required solution to the system (5.4), i.e., $x_{1}^{*}=-1$ and $x_{2}^{*}=2$.

Example 5.4. In this example, we consider the following system

$$
\left[\begin{array}{rrr}
0 & 0 & 1 \\
2 & 0 & -1 \\
1 & 2 & 3
\end{array}\right] \quad\left[\begin{array}{l}
x_{1} \\
x_{2} \\
x_{3}
\end{array}\right]=\left[\begin{array}{l}
1 \\
3 \\
1
\end{array}\right]
$$

The coefficient matrix of this system has the first two leading principal minors equal to zero. Consequently, the first two pivots, in the Gauss transformations, are zero. We solve the system without pivoting by substituting the zero pivot by $1^{-1}$, where necessary.

Let us show how the exact computations are executed:

$$
\begin{aligned}
& {\left[\begin{array}{rrr|r}
0 & 0 & 1 & 1 \\
2 & 0 & -1 & 3 \\
1 & 2 & 3 & 1
\end{array}\right] \rightarrow\left[\begin{array}{ccc|c}
1 & 0 & 1 & 1 \\
0 & 0 & -21-1 & -21+3 \\
0 & 2 & -1(1)+3 & -1+1
\end{array}\right] \rightarrow} \\
& {\left[\begin{array}{ccc|c}
1 & 0 & 1 & 1 \\
0 & 1 & -2(1)^{2}-(1) & -2(1)^{2}+31 \\
0 & 2 & -11+3 & -(1)+1
\end{array}\right] \rightarrow\left[\begin{array}{ccc|c}
1 & 0 & 1 & 1 \\
0 & 1 & -2(1)^{2}-1 & -2(1)^{2}+31 \\
0 & 0 & 4\left(1^{2}+(1)+3\right. & \left.41^{2}-71\right)+1
\end{array}\right] \rightarrow} \\
& {\left[\begin{array}{ccc|c}
1 & 0 & 1 & 1 \\
0 & 1 & -2(1)^{2}-(1) & -2(1)^{2}+31 \\
0 & 0 & 1 & \frac{4\left(1^{2}-7(1)+1\right.}{4(1)^{2}+(1)+3}
\end{array}\right] \rightarrow\left[\begin{array}{lll|c}
1 & 0 & 0 & \frac{8(1)^{2}+2(1)}{\left.4(1)^{2}+1\right)+3} \\
0 & 1 & 0 & \frac{-8(1)^{2}+10(1)}{\left.4(1)^{2}+1\right)+3} \\
0 & 0 & 1 & \frac{4(1)^{2}-7(1)+1}{\left.4(1)^{2}+1\right)+3}
\end{array}\right] .}
\end{aligned}
$$

It is easy to see that the finite parts of the numbers

$$
\begin{aligned}
& \tilde{x}_{1}^{*}=\frac{8(1)^{2}+21}{4(1)^{2}+(1)+3}=2-\frac{6}{4(1)^{2}+(1)+3}, \\
& \tilde{x}_{2}^{*}=\frac{-8(1)^{2}+10(1)}{4(1)^{2}+(1)+3}=-2+\frac{12(1)+6}{4(1)^{2}+(1)+3}, \\
& \tilde{x}_{3}^{*}=\frac{4(1)^{2}-71+1}{4(1)^{2}+(1)+3}=1-\frac{8(1)+2}{4(1)^{2}+(1)+3}
\end{aligned}
$$


provide the solution $x_{1}^{*}=2, x_{2}^{*}=-2$, and $x_{3}^{*}=1$ to the original unperturbed system (5.5).

In this procedure we have introduced the number $1^{-1}$ two times. As a result, we have obtained expressions where the maximal power of grossone is equal to 2 and there are rational expressions depending on grossone, as well. By reasoning analogously to Example 5.4, when we execute divisions, in the obtained results it is not necessary to store the parts of the type $c_{p} \mathbb{1}^{p}, p<-2$, because in any way the result of their successive multiplication with the numbers of the type $c_{2}\left(1^{2}\right.$ will give finite exponents less than zero. That is, numbers with these exponents will be infinitesimals that are not interesting for us in this computational context. Thus, by using the positional numeral system (4.16), (4.17), we obtain

$$
\left[\begin{array}{ccc|c}
1 & 0 & (1) & 1 \\
0 & 1 & -2(1)^{2}-(1) & -2(1)^{2}+3(1) \\
0 & 0 & 1 & \frac{4(1)^{2}-7(1)+1}{4(1)^{2}+(1)+3}
\end{array}\right] \rightarrow\left[\begin{array}{ccc|c}
1 & 0 & 1 & 1 \\
0 & 1 & -2(1)^{2}-(1) & -2(1)^{2}+3(1) \\
0 & 0 & 1 & 1(1)^{0}-2(1)^{-1}
\end{array}\right] .
$$

Note that the number $11^{0}-2\left(1^{-1}\right.$ does not contain the part of the type $c_{-2}{ }^{(1)}-2$ because the coefficient $c_{-2}$ obtained after the executed division is such that $c_{-2}=0$. Then we proceed as follows

$$
\left[\begin{array}{lll|c}
1 & 0 & 1 & 1 \\
0 & 1 & 0 & -2 \\
0 & 0 & 1 & 11^{0}-2\left(1^{-1}\right.
\end{array}\right] \rightarrow\left[\begin{array}{lll|c}
1 & 0 & 0 & 2 \\
0 & 1 & 0 & -2 \\
0 & 0 & 1 & 11^{0}-2(1)^{-1}
\end{array}\right]
$$

The obtained solutions $x_{1}^{*}=2$ and $x_{2}^{*}=-2$ have been obtained exactly without infinitesimal parts and $x_{3}^{*}=1$ is derived from the finite part of $11^{0}-21^{-1}$.

We conclude this subsection by mentioning that zero pivots in the matrix are substituted dynamically by $(1)^{-1}$. Thus, the number of the introduced infinitesimals $\mathbb{1}^{-1}$ depends on the number of zero pivots.

5.3. Infinite penalty coefficients in constrained non-linear optimization. In papers $[32,33]$, a number of interesting applications of (1) in linear and non-linear optimization have been proposed. One of them consists of using infinite (1)-based penalty coefficients in order to transform a constrained optimization problem into an unconstrained one. Traditionally, this is done by using a sequence of finite penalty coefficients and for each coefficient the corresponding unconstrained problem should be solved. Therefore, there arises the necessity to solve a sequence of unconstrained problems and solution to each unconstrained problem gives an approximation to the solution to the original constrained problem. The following example taken form [32] illustrates the advantages provided by (1) in this situation. In fact, instead of getting an approximate solution obtained after solving a series of unconstrained problems the authors of [32] show that only one unconstrained problem can be solved thanks to grossone and solution to this problem provides the exact solution to the original constrained problem. 
Example 5.5. Let us consider the following quadratic 2-dimensional optimization problem with a single linear constraint proposed by Liuzzi in 2007:

$$
\begin{gathered}
\min _{x} \frac{1}{2} x_{1}^{2}+\frac{1}{6} x_{2}^{2} \\
\text { subject to } \quad x_{1}+x_{2}=1
\end{gathered}
$$

One of the traditional ways to solve this problem is to construct the corresponding unconstrained optimization problem using a finite penalty coefficient $P$ as follows

$$
\min _{x} \frac{1}{2} x_{1}^{2}+\frac{1}{6} x_{2}^{2}+\frac{P}{2}\left(1-x_{1}-x_{2}\right)^{2}
$$

Then, different values of $P$ should be taken into consideration. For instance, let us take $P=20$ and write down the first order optimality conditions

$$
\left\{\begin{array}{c}
x_{1}-20\left(1-x_{1}-x_{2}\right)=0 \\
\frac{1}{3} x_{2}-20\left(1-x_{1}-x_{2}\right)=0 .
\end{array}\right.
$$

The solution to this system of linear equations is the stationary point of the unconstrained problem (5.7) with $P=20$, namely, it is

$$
\tilde{x}_{1}^{*}(20)=\frac{20}{81}, \quad \tilde{x}_{2}^{*}(20)=\frac{60}{81}
$$

and it is not clear how to obtain from (5.8) the solution to the original constrained problem (5.6). Thus, another penalty coefficient $P$ should be taken, the problem (5.7) should be solved again in order to get a new approximation to the solution of (5.6). This procedure should be repeated several times until a satisfactory approximation will be obtained.

In the Infinity Computer framework, the authors of [32] propose to take $P=(1$. The corresponding first order optimality conditions in this case are

$$
\left\{\begin{array}{c}
x_{1}-1\left(1-x_{1}-x_{2}\right)=0 \\
\frac{1}{3} x_{2}-1\left(1-x_{1}-x_{2}\right)=0 .
\end{array}\right.
$$

Therefore, the stationary point of the unconstraint problem (5.7) with $P=(1$ is

$$
\tilde{x}_{1}^{*}=\frac{11}{1+41}, \quad \tilde{x}_{2}^{*}=\frac{3(1)}{1+41}
$$

and after division we get

$$
\tilde{x}_{1}^{*}=\frac{1}{4}-(1)^{-1}\left(\frac{1}{16}-\frac{1}{64} 1^{-1}+\ldots\right), \quad \tilde{x}_{2}^{*}=\frac{3}{4}-(1)^{-1}\left(\frac{3}{16}-\frac{3}{64}\left(1^{-1}+\ldots\right) .\right.
$$

The authors of [32] have proved that the finite parts of $\tilde{x}_{1}^{*}$ and $\tilde{x}_{2}^{*}$ give the exact solution $x^{*}=\left(\frac{1}{4}, \frac{3}{4}\right)$ to the original constrained problem (5.6). 
5.4. Lexicographic multi-objective linear programming. Engineering applications often lead to optimization problems where several objectives should be satisfied. An important class of problems of this kind is lexicographic multi-objective problems where the first objective is incomparably more important than the second one which, in its turn, is incomparably more important than the third one, etc. In case each of the objectives is represented by a linear function under linear constraints, Lexicographic Multi-Objective Linear Programming (LMOLP) problems are considered. Traditionally LMOLP problems are solved in two different ways (see, e.g., [55, 80, 107, 109]). The first one called preemptive approach consists of solving a sequence of single-objective linear programming (LP) problems, where new constraints are added to the subsequent LP problem once an optimal solution to the previous problem has been found. Clearly, this approach is time consuming. The second approach known as nonpreemptive one (see [107]) transforms LMOLP into a single-objective LP problem by using a weighted sum of the objectives. This approach has the difficulty to find the weights which guarantee, a-priori, the equivalence of the resulting single-objective problem with the original multi-objective one. In practice, determining such weights is a tricky task and it can be even more time consuming then the preemptive approach.

Following an idea of the (1)-based lexicographic ordering proposed in [100] it has been shown in [20] how to transform LMOLP into a single-objective LP problem. This is done by multiplying the most important objective by 1 , the second by $1^{-1}$, the third by $1^{-2}$ etc., where $\left(^{-i+1}, 1 \leq i \leq r\right.$, are infinitesimals and $r$ is a finite number of objectives. It can be shown then (see [20] for a detailed discussion) that after this transformation the resulting single-objective LP problem can be solved only once by using a simplex-like method working with (1)-based numbers that can include infinitesimal parts. The overall advantage of this approach consists of the possibility to solve only one LP problem, without the need to look for correct finite weights that should be provided to an algorithm if the traditional nonpreemptive scheme is applied.

More formally, let us consider the following LMOLP problem where LexMax denotes Lexicographic Maximum and means that the first objective is much more important than the second, which is, in turn, much more important than the third one, and so on:

$$
\begin{gathered}
\text { LexMax } c^{1} \cdot x, c^{2} \cdot x, \ldots, c^{r} \cdot x \\
\text { subject to } A x=b, x \geq 0
\end{gathered}
$$

where $r$ is the number of objectives to optimize, $c^{i}, i=1, \ldots, r$, are $n$-dimensional row vectors, $x$ and $b$ are $n$-dimensional column vectors, $A$ is a full-rank $m \times n$ matrix and . denotes the standard scalar product between two vectors. Vectors $b, c^{i}, i=1, \ldots, r$, and matrix $A$ have purely finite components. As in any LP problem, the domain of (5.9) is a polytope $S$ and it is assumed hereinafter that $S$ is bounded and non-empty.

It has been shown in [107] that there always exists a finite scalar $M$ such that the solution to the LMOLP problem (5.9) can be found by solving only one single-objective LP problem having the following form

$$
\begin{gathered}
\max \bar{c} \cdot x \\
\text { subject to } A x=b, x \geq 0
\end{gathered}
$$


where

$$
\bar{c}=\sum_{i=1}^{r} c^{i} M^{-i+1} .
$$

This is a powerful theoretical result. However, from the computational point of view finding the value of $M$ is not a trivial task. Finding an appropriate finite value of $M$ and solving the resulting LP problem can be more time consuming than solving the original problem (5.9) following the preemptive approach. Indeed, the preemptive scheme requires solving $r$ linear programming problems only in the worst case and, in addition, it does not require the computation of $M$.

Let us describe now the nonpreemptive (1)-based scheme following [20] where the problem

$$
\begin{gathered}
\max \hat{c} \cdot x, \\
\text { subject to } A x=b, x \geq 0
\end{gathered}
$$

is constructed. In (5.12), $\hat{c}$ is built using (1)-based weights

$$
\hat{c}=\sum_{i=1}^{r} c^{i}{ }^{-i+1}
$$

and, therefore, it follows that

$$
\hat{c} \cdot x=\left(c^{1} \cdot x\right) 1^{0}+\left(c^{2} \cdot x\right) 1^{-1}+\ldots+\left(c^{r} \cdot x\right) 1^{-r+1},
$$

where (5.14) can be equivalently written in the extended form as

$$
\left.\hat{c} \cdot x=\left(c_{1}^{1} x_{1}+\ldots+c_{n}^{1} x_{n}\right) 1^{0}+\left(c_{1}^{2} x_{1}+\ldots+c_{n}^{2} x_{n}\right) \mathbb{1}^{-1}+\ldots+\left(c_{1}^{r} x_{1}+\ldots+c_{n}^{r} x_{n}\right)^{1}\right)^{-r+1} .
$$

The crucial advantage of (5.12) with respect to the traditional nonpreemptive scheme is that $\hat{c}$ does not involve any unknown. More precisely, it does not require the search of a finite value $M$ from (5.10), (5.11). In [20], a simplex-like algorithm called gross-simplex was proposed. It is able to work with (1)-based numerals and, therefore, can solve (5.12) numerically.

5.5. Numerical methods using infinitesimals for solving ODEs. Numerical solutions to Ordinary Differential Equations (ODEs) are required very often in practical problems and there exists a huge number of numerical methods proposed to solve ODEs on conventional computers (see, e.g., [12, 13, 18, 47, 48, 52, 54, 73] and references given therein). Let us consider the following initial value problem

$$
y^{\prime}(x)=f(x, y), y\left(x_{0}\right)=y_{0}, x_{0}=a, x \in[a, b],
$$

and suppose that $f(x, y)$ is a 'black-box' function, i.e., $f(x, y)$ is given by a computer procedure and the analytical representation of $f(x, y)$ is unknown to the person who solves (5.15).

Many numerical methods for solving ODEs require the computation of the derivative of the unknown function $y(x)$ at some specific points. In particular, this is the case with 
methods based on Taylor expansion. Usually derivatives are approximated by using automatic differentiation or numerical approximations. Automatic differentiation techniques make use of specific, often symbolic, tools based on the elementary functions involved (see [5]). As was already mentioned, numerical differentiation, especially when higher order derivatives are necessary, suffers of numerical instability and is not able to reach a higher precision. Finite difference formulas are, in fact, ill-conditioned, moreover the cancellation error produces a value of zero if $h$ is small. As was shown in Section 5.1, the use of the Infinity Computer allows to compute exact derivatives using the infinitesimal perturbation equal to $1^{-1}$. This feature, besides preventing the above mentioned ill-conditioning phenomenon, is particularly appealing in the case where the function to be differentiated is a 'black-box'. Let us show now how this fact can be used for solving the problem (5.15).

The simplest algorithm to solve (5.15) is probably the explicit Euler Method (see, e.g., [13]). At each step it constructs a linear approximation of $y(x)$ starting from the initial point $\left(x_{0}, y\left(x_{0}\right)\right)$. The $(n+1)$ th step of the Euler algorithm describes how to move from the point $x_{n}$ to $x_{n+1}=x_{n}+h, n \geq 0$, and is executed as follows

$$
y_{n+1}=y_{n}+h f\left(x_{n}, y_{n}\right) .
$$

Let us denote by $y^{(k)}\left(x_{i}\right)$ an estimate of the $k$-th derivative of the solution $y(x)$ at the point $x_{i}$. It has been shown in [98] that in order to calculate the $k$-th derivative of the unknown solution $y(x)$ at the point $x_{i}, k$ infinitesimals steps from the point $x_{i}$ using the Euler formula with $h=(1)^{-1}$ should be executed as follows

$$
\begin{gathered}
y_{i 1}=y_{i}+(1)^{-1} f\left(x_{i}, y_{i}\right), \quad y_{i 2}=y_{i 1}+1^{-1} f\left(x_{i}+(1)^{-1}, y_{i 1}\right), \ldots \\
y_{i k}=y_{i, k-1}+\left(1^{-1} f\left(x_{i}+(k-1)\right)^{-1}, y_{i, k-1}\right) .
\end{gathered}
$$

Then, since approximations of the derivatives can be obtained by the forward differences $\Delta_{h}^{j}, 1 \leq j \leq k$, the value of $h$ is taken equal to $1^{-1}$ and it follows that

$$
\Delta_{\Phi^{-1}}^{k}=\sum_{j=0}^{k}(-1)^{j}\left(\begin{array}{l}
j \\
k
\end{array}\right) y_{x_{i}+(k-j) \Phi^{-1}},
$$

where $\left(\begin{array}{l}j \\ k\end{array}\right)$ is a binomial coefficient and

$$
y^{(k)}\left(x_{i}\right) \approx \frac{\Delta_{\mathbb{\Phi}^{-1}}^{k}}{(1)^{-k}}+O\left(\mathbb{1}^{-1}\right) .
$$

Then, the main observation consists of the fact that, since the error in (5.18) has the order equal to $1^{-1}$, the finite part of the value $\frac{\Delta_{\Phi^{-1}}^{k}}{(1)^{-k}}$ gives us the exact derivative $y^{(k)}\left(x_{i}\right)$ (for a more detailed description of the computation of derivatives for solving ODEs see [98]).

Since we are able to compute values of $k$ derivatives of the function $y(x)$ at a generic point $x_{0}$, we can estimate $y(x)$ in a neighborhood of the point $x_{0}$ by its Taylor expansion

$$
y(x) \approx \hat{y}(x)=y_{0}+\sum_{i=1}^{k} \frac{y^{(i)}\left(x_{0}\right)}{i !}\left(x-x_{0}\right)^{i} .
$$


In cases where the radius of convergence of the Taylor expansion $\hat{y}(x)$ from (5.19) covers the whole interval $[a, b]$ of our interest, then there is no necessity to execute several steps with a finite value of $h$. In fact, thanks to the exact derivatives calculated numerically on the Infinity Computer the function $y(x)$ can be approximated in the neighborhood of the initial point $x_{0}$ by its Taylor expansion $\hat{y}(x)$ with an order $k$ depending on the desired accuracy and then $\hat{y}(x)$ can be evaluated at any point $x \in[a, b]$. This method is called Taylor for the Infinity Computer (TIC) hereinafter. This method does not assume the execution of several iterations with a finite step $h$. The following example shows how this procedure works.

Example 5.6. Let us consider the problem

$$
y^{\prime}(x)=x-y, \quad y(0)=1,
$$

taken from [1]. Its exact solution is

$$
y(x)=x-1+2 e^{-x} .
$$

Let us construct the Taylor expansion of $y(x)$ at the initial point $x_{0}$ by applying formula (5.16) with $h=1^{-1}$ to calculate $y_{1}$ and $y_{2}$ :

$$
\begin{gathered}
y_{1}=1+\left(\mathbb{1}^{-1} \cdot(0-1)=1-\left(\mathbb{1}^{-1},\right.\right. \\
y_{2}=1-\left(\mathbb{1}^{-1}+\mathbb{1}^{-1}\left(\mathbb{1}^{-1}-1+\left(\mathbb{1}^{-1}\right)=1-2 \mathbb{1}^{-1}+2 \mathbb{1}^{-2} .\right.\right.
\end{gathered}
$$

We have now the values $y_{0}, y_{1}$, and $y_{2}$. Thus, we can calculate $\triangle_{\Phi^{-1}}^{2}$ as follows

$$
\triangle_{\mathbb{\Phi}^{-1}}^{2}=y_{0}-2 y_{1}+y_{2}=1-2+21^{-1}+1-21^{-1}+2\left(1^{-2}=21^{-2} .\right.
$$

Thus, $c_{-2}=2$. Let us now verify the obtained result and calculate the exact derivative $y^{\prime \prime}(0)$ using (5.21). Then we have $y^{\prime \prime}(x)=2 e^{-x}$, and $y^{\prime \prime}(0)=2$, i.e., $c_{-2}=y^{\prime \prime}(0)$.

Let us proceed and calculate $y_{3}$ as follows

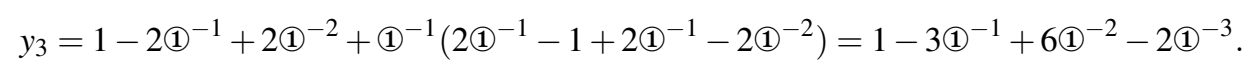

Thus, we can compute

$$
\begin{gathered}
\triangle_{\Phi^{-1}}^{3}=-y_{0}+3 y_{1}-3 y_{2}+y_{3}= \\
-1+3\left(1-\left(1^{-1}\right)-3\left(1-21^{-1}+2\left(1^{-2}\right)+1-3\left(1^{-1}+61^{-2}-2(1)^{-3}=-2(1)^{-3} .\right.\right.\right.
\end{gathered}
$$

We can see that $c_{-3}=-2$. The exact derivative obtained from (5.21) is $y^{(3)}(x)=-2 e^{-x}$. As a consequence, we have $y^{(3)}(0)=-2$, i.e., $c_{-3}=y^{(3)}(0)$.

To calculate $\triangle_{\mathbb{Q}^{-1}}^{4}$ we compute $y^{(4)}(0)$ and obtain

$$
\begin{aligned}
& y_{4}=1-3\left(1^{-1}+61^{-2}-2\left(1^{-3}+\left(1^{-1}\left(31^{-1}-1+31^{-1}-6(1)^{-2}+2(1)^{-3}\right)=\right.\right.\right. \\
& 1-41^{-1}+12(1)^{-2}-81^{-3}+21^{-4} \\
& \triangle_{\Phi^{-1}}^{4}=y_{0}-4 y_{1}+6 y_{2}-4 y_{3}+y_{4}=1-4\left(1-\mathbb{1}^{-1}\right)+6\left(1-21^{-1}+21^{-2}\right)-
\end{aligned}
$$




\begin{tabular}{|c|c|c|c|c|c|}
\hline \multirow{2}{*}{ Test problem } & $y \_$RK4 & $\varepsilon \_$RK4 & $y_{-}$TIC & $\varepsilon_{-}$TIC & $N_{-}$TIC \\
\hline 1 & 0.837462 & $-8.62538 \mathrm{e}-009$ & 0.837462 & $-5.91687 \mathrm{e}-009$ & 6 \\
\hline 2 & 1.242806 & $8.11157 \mathrm{e}-009$ & 1.242806 & $4.19151 \mathrm{e}-009$ & 6 \\
\hline 3 & 1.221403 & $4.12685 \mathrm{e}-009$ & 1.221403 & $2.13248 \mathrm{e}-009$ & 6 \\
\hline 4 & 1.221403 & $3.89834 \mathrm{e}-008$ & 1.221403 & $2.13248 \mathrm{e}-009$ & 6 \\
\hline 5 & 1.491817 & $1.27726 \mathrm{e}-007$ & 1.491817 & $1.13693 \mathrm{e}-008$ & 7 \\
\hline 6 & 0.135416 & $-5.96529 \mathrm{e}-004$ & 0.135379 & $-3.24420 \mathrm{e}-004$ & 10 \\
\hline 7 & 36.154673 & $-8.16405 \mathrm{e}-005$ & 36.149608 & $5.84540 \mathrm{e}-005$ & 9 \\
\hline 8 & 35.968459 & $-8.18293 \mathrm{e}-005$ & 35.963409 & $5.85817 \mathrm{e}-005$ & 9 \\
\hline 9 & 1.239230 & $-5.78803 \mathrm{e}-009$ & 1.239230 & $-4.08211 \mathrm{e}-009$ & 10 \\
\hline 10 & 0.781397 & $-1.76949 \mathrm{e}-009$ & 0.781397 & $7.94128 \mathrm{e}-011$ & 7 \\
\hline 11 & 1.153846 & $8.98577 \mathrm{e}-009$ & 1.153846 & $4.09600 \mathrm{e}-009$ & 11 \\
\hline 12 & 0.472441 & $2.95775 \mathrm{e}-010$ & 0.472441 & $-1.60782 \mathrm{e}-010$ & 10 \\
\hline
\end{tabular}

Table 5.1. Results of a comparison on 12 test problems taken from the literature of the TIC method with the Runge-Kutta method of the fourth order that executes 20 evaluations of $f(x, y)$ to reach the accuracy $\varepsilon \_$RK4

$$
\begin{aligned}
& -4\left(1-31^{-1}+6\left(1^{-2}-2\left(1^{-3}\right)+1-41^{-1}+121^{-2}-81^{-3}+21^{-4}=\right.\right. \\
& -4\left(1-31^{-1}+6(1)^{-2}-2\left(1^{-3}\right)+1-41^{-1}+12\left(1^{-2}-8(1)^{-3}+2(1)^{-4}=21^{-4}\right. \text {. }\right.
\end{aligned}
$$

Therefore, we obtain that $c_{-4}=y^{(4)}(0)=2$.

Thus, four steps of the explicit Euler method with the infinitesimal step $h=(1)^{-1}$ have been executed. As a result, the first five exact items of the Taylor expansion of $y(x)$ in the neighborhood of $x_{0}=0$ can be written:

$$
y(x)=x-1+2 e^{-x} \approx 1-x+x^{2}-\frac{x^{3}}{3}+\frac{x^{4}}{12} .
$$

By a complete analogy it is possible to obtain additional terms in the expansion that correspond to higher derivatives of $y(x)$.

Table 5.5 presents results of numerical experiments from [105] executed on a class of 12 test functions taken from the literature. The method TIC is compared over the interval $[0,0.2]$ with the Runge-Kutta method of the fourth order (RK4) with the integration step $h=0.04$, i.e., to obtain an approximation at the point $x=0.2$ the method RK4 executes 5 steps and 20 evaluations of the function $f(x, y)$ from (5.15). After the results for RK4 had been obtained, the TIC method was applied to each of 12 problems. The method TIC stopped when the accuracy $\varepsilon_{-}$TIC at the point $x=0.2$ was better than the accuracy $\varepsilon \_$RK4 of the method RK4. The last column, $N_{-}$TIC, in Table 5.5 presents the number of evaluations of $f(x, y)$ executed by the TIC to reach the accuracy $\varepsilon_{-}$TIC. In other words, it shows the number of infinitesimal steps executed by the TIC that is equal to the number of exact derivatives calculated by this method. The respective solutions $y_{-}$RK4 and $y_{-}$TIC are also shown in the table. For the considered problem the TIC method executes fewer evaluations of $f(x, y)$, in comparison with the Runge-Kutta method.

More sophisticated algorithms developing the idea of the usage of the infinitesimal stepsize equal to $1^{-1}$ can be found in $[2,74,105]$. In particular, it is shown in these papers how to construct methods mixing finite and infinitesimal steps of integration. 


\section{Traditional mathematical objects viewed from the new methodological positions}

6.1. Infinite sequences and Turing machines. We start this section by reminding traditional definitions of infinite sequences and subsequences. An infinite sequence $\left\{a_{n}\right\}, a_{n} \in$ $A, n \in \mathbb{N}$, is a function having as the domain the set of natural numbers, $\mathbb{N}$, and as the codomain a set $A$. A subsequence is obtained from a sequence by deleting some (or possibly none) of its elements. In a sequence $a_{1}, a_{2}, \ldots, a_{n-1}, a_{n}$ the number $n$ is the number of elements of the sequence. Traditionally, only finite values of $n$ are considered. Grossone-based numerals give us the possibility to observe infinite numbers and, therefore, to see not only the initial elements of an infinite sequence $a_{1}, a_{2}, \ldots$ but also its final part $\ldots, a_{n-1}, a_{n}$ where $n$ can assume different infinite values. The following result then holds.

Proposition 6.1. The number of elements of any infinite sequence is less than or equal to 1 .

Proof. The IUA states that the set of natural numbers, $\mathbb{N}$, has 1 elements. Thus, by the above definition, any sequence having $\mathbb{N}$ as the domain has (1) elements.

Any subsequence is obtained by deleting some (or possibly none) of the (1) elements from a sequence. Thus, it will have at most grossone elements.

One of the immediate consequences of the understanding of this result is that any sequential process can have at most (1) elements. Also recall that, as was emphasized in Postulate 1, we are able to execute only a finite number of operations. Thus, in any infinite sequential process only a finite number of its elements can be observed and which elements can be observed depends on the chosen numeral system. Let us illustrate this assertion by the following example.

Example 6.2. Let us consider the set, $\widehat{\mathbb{N}}$, of extended natural numbers. Then starting from the number 1 , it is possible to arrive at most to (1)

$$
\underbrace{1,2,3,4, \ldots \text { (1) }-2,(1)-1,(1)}_{\text {(1) elements }},(1)+1,(1)+2,(1)+3, \ldots
$$

Starting from 2 it is possible to arrive at most to $1+1$

$$
1, \underbrace{2,3,4, \ldots \text { (1) }-2,(1)-1,(1),(1)+1}_{(1) \text { elements }},(1)+2,(1)+3, \ldots
$$

Starting from 3 it is possible to to arrive at most to $1+2$

$$
1,2, \underbrace{3,4, \ldots \text { (1) }-2,(1)-1,(1),(1)+1,(1)+2}_{(1) \text { elements }},(1)+3, \ldots
$$

Of course, since we have postulated that our possibilities to express numerals are finite, it depends on the chosen numeral system which numbers among (1) members of these processes we can observe. 
It becomes appropriate now to define a complete sequence as an infinite sequence containing exactly (1) elements. For example, the sequence of natural numbers is complete, the sequences of even and odd natural numbers are not complete. Thus, the IUA yields a more precise description of infinite sequences. To define a sequence $\left\{a_{n}\right\}$ it is not sufficient just to give a formula for $a_{n}$, we should determine (as it happens for sequences having a finite number of elements) the first and the last elements of the sequence or the first element and the number of elements. If the number of the first element is equal to one, we can use the record $\left\{a_{n}: k\right\}$ where $a_{n}$ is, as usual, the general element of the sequence and $k$ is the number (that can be finite or infinite) of members of the sequence.

Example 6.3. Let us consider the following three sequences, $\left\{a_{n}\right\},\left\{b_{n}\right\}$, and $\left\{c_{n}\right\}$ :

$$
\begin{aligned}
& \left\{a_{n}\right\}=\{5, \quad 10, \ldots 5(1-1), \quad 5(1)\}, \\
& \left\{b_{n}\right\}=\left\{5, \quad 10, \quad \ldots \quad 5\left(\frac{21}{5}-1\right), \quad 5 \cdot \frac{21}{5}\right\}, \\
& \left\{c_{n}\right\}=\left\{5, \quad 10, \quad \ldots \quad 5\left(\frac{41}{5}-1\right), \quad 5 \cdot \frac{41}{5}\right\} .
\end{aligned}
$$

They have the same general element $a_{n}=b_{n}=c_{n}=5 n$ but they are different because the first sequence has 1 elements and is thus complete, the other two sequences are not complete: $\left\{b_{n}\right\}$ has $\frac{2(1)}{5}$ elements and $\left\{c_{n}\right\}$ has $\frac{4(1)}{5}$ members.

In connection with this definition of infinite sequences the following natural question arises. Suppose that we have two sequences, for example, $\left\{b_{n}: \frac{2(1)}{5}\right\}$ and $\left\{c_{n}: \frac{41}{5}\right\}$ from (6.4) and (6.5). Can we create a new sequence, $\left\{d_{n}: k\right\}$, composed from both of them, for instance, as it is shown below

$$
b_{1}, b_{2}, \ldots b_{\frac{2 \mathbb{N}}{5}-2}, b_{\frac{2 \mathbb{T}}{5}-1}, b_{\frac{2 \mathbb{N}}{5}}, c_{1}, c_{2}, \ldots c_{\frac{4 \mathbb{1}}{5}-2}, c_{\frac{4 \mathbb{N}}{5}-1}, c_{\frac{4 \mathbb{1}}{5}}
$$

and which will be the value of the number of its elements $k$ ?

The answer is 'no' because due to the definition of the infinite sequence, a sequence can be at maximum complete, i.e., it cannot have more than (1) elements. Starting from the element $b_{1}$ we can arrive at maximum to the element $c_{\frac{3 \Phi}{5}}$ being the element number (1) in the sequence $\left\{d_{n}: k\right\}$ which we try to construct. Therefore, $k=1$ and

$$
\underbrace{b_{1}, \ldots b_{\frac{2 \Phi}{5}}, c_{1}, \ldots c_{\frac{3 \Phi}{5}}}_{\text {(1) elements }}, \underbrace{c_{\frac{3 \Phi}{5}+1}, \ldots c_{\frac{4 \Phi}{5}}}_{\frac{1}{5} \text { elements }} .
$$

The remaining members of the sequence $\left\{c_{n}: \frac{4(1)}{5}\right\}$ will form the second sequence, $\left\{g_{n}: l\right\}$ having $l=\frac{4(1)}{5}-\frac{3(1)}{5}=\frac{1}{5}$ elements. Thus, we have formed two sequences, the first of them is complete and the second is not. This situation has also its granary interpretation. Namely, if one has many seeds, in a sack it is possible to put only the quantity that can enter in the sack. Then, if there remain other seeds, it is necessary to take another sack.

The possibility to see the final elements of sequences is very important in several areas of Mathematics and Computer Science. For instance, this allows us to distinguish Turing machines with infinite tapes having different lengths (see [103, 104]). 
Suppose that we have a Turing machine with an infinite tape that contains an output written using symbols $\{0,1, \ldots b-2, b-1\}$ with a finite radix $b$. The traditional point of view does not allow us to distinguish neither tapes having different infinite lengths nor machines using different alphabets, i.e., $\{0,1, \ldots B-2, B-1\}$ with $B \neq b$. The question of the possibility to have different infinite tapes is not discussed and it is supposed that machines with any output alphabets have the same computational power if their tapes are infinite. This happens because traditional numeral systems used to describe Turing machines do not allow us to see these differences. The (1)-based numeral system offers such a possibility thus providing a tool to describe Turing machines in a more precise way.

In the (1)-based framework, it is not sufficient to say that the tape is infinite. It is necessary to define the infinite length of the tape explicitly. As an example, let us consider a Turing machine whose tape is a sequence of (1) squares. Output sequences are written on the tape using symbols from an output alphabet, let it be again $\{0,1, \ldots b-2, b-1\}$ with a finite $b$. The importance of the discussion of infinite sequences provided above for Turing machines becomes now clear: the output sequences of symbols being sequences cannot have more than (1) elements.

Moreover, we can make a more accurate analysis and count the precise number of infinite output sequences of symbols that the machine can produce. It is obvious that its outputs can be viewed as numerals in the positional numeral system with the finite radix $b$

$$
\left(a_{1} a_{2} \ldots a_{\Phi-1} a_{\Phi}\right)_{b}, \quad a_{i} \in\{0,1, \ldots b-2, b-1\}, \quad 1 \leq i \leq 1 .
$$

This means that we have (1) positions that can be filled in with $b$ symbols each, i.e., this machine called hereinafter $T_{1}$ can produce $b^{\bowtie}$ different outputs. Then, if we consider another machine, $T_{2}$, having the tape with (1)-1 positions and outputs written using the same base, $b$, the number of its outputs is $b^{\circledR-1}<b^{\circledR}$ and each of them is one position shorter than outputs of $T_{1}$. Moreover, if we consider the third machine, $T_{3}$, having the tape with (1) positions and outputs written using a base $B>b$, the number of its outputs is $B^{\Phi}>b^{\bowtie}$. In other words, the machine $T_{3}$ is more powerful then the machine $T_{1}$ that, in its turn, is more powerful than the machine $T_{2}$. Let us give a couple of more detailed illustrations.

Example 6.4. We start by considering a Turing machine $T_{4}$ working with the alphabet $\{0,1,2\}$, the tape with $1 / 2$ positions, and computing the following output

$$
\underbrace{0,1,2,0,1,2,0,1,2, \ldots 0,1,2,0,1,2}_{(1 / 2 \text { positions }} .
$$

Then a Turing machine $T_{5}$ working with the output alphabet $\{0,1\}$ and the tape with (1)/2 positions cannot produce a sequence of symbols computing (6.7). In fact, since the numeral 2 does not belong to the alphabet $\{0,1\}$ it should be coded by more than one symbol. One of codifications using the minimal number of symbols in the alphabet $\{0,1\}$ necessary to code numbers $0,1,2$ is $\{00,01,10\}$. Then the output corresponding to (6.7) and computed in this codification should be

$$
00,01,10,00,01,10,00,01,10, \ldots 00,01,10,00,01,10 .
$$


Since the output (6.7) contains (1/2 positions, the output (6.8) should contain (1) positions. However, by the definition of $T_{5}$ it can produce outputs that have only (1)/2 positions.

Example 6.5. Let us now consider a Turing machine $T_{6}$ working with the alphabet $\{0,1,2\}$ as $T_{4}$ but whose infinite tape is one position longer than the tape of $T_{4}$, i.e., it has $1 / 2+1$ positions, and $T_{6}$ computes the following output

$$
\underbrace{0,1,2,0,1,2,0,1,2, \ldots 0,1,2,0,1,2,0}_{(\mathbb{1} / 2+1 \text { positions }} .
$$

Then there is no Turing machine working with the output alphabet $\{0,1\}$ and coding the numbers $0,1,2$ as $\{00,01,10\}$ such that it is able to compute the output corresponding to (6.9) in this codification. The proof is very easy and is based on the fact that infinite sequences cannot have more than (1) elements. Since the output (6.9) contains (1/2+1 positions, the output

$$
00,01,10,00,01,10,00,01,10, \ldots 00,01,10,00,01,10,00 .
$$

should contain (1) +2 positions. However, infinite sequences cannot have more than (1) elements.

Significantly more sophisticated results for deterministic and non-deterministic Turing machines can be found in [103, 104].

6.2. From divergent series to sums with a fixed infinite number of addends. Suppose that we consider two divergent series

$$
S_{1}=\sum_{i=1}^{\infty} a_{i}, \quad S_{2}=\sum_{i=1}^{\infty} b_{i} .
$$

The traditional analysis can say a little about them and it does not allow us to execute numerical computations with them, e.g., such operations as $\frac{S_{2}}{S_{1}}$ and $S_{2}-S_{1}$ are not defined.

The (1)-based methodology allows us to express not only different finite numbers but also different infinite numbers. Thus, such records as $S_{1}=a_{1}+a_{2}+\ldots$ or $\sum_{i=1}^{\infty} a_{i}$ become imprecise (to continue the analogy with Pirahã the record $\sum_{i=1}^{\infty} a_{i}$ is viewed as a kind of $\sum_{i=1}^{\text {many }} a_{i}$ ). It is therefore necessary to indicate explicitly the infinite numbers, $k$ and $n$, being the number of summands in the respective sums $S_{1}$ and $S_{2}$ from (6.10). Thus, we get more precise definitions of these sums where the infinite numbers of addends are specified

$$
S_{1}(k)=a_{1}+a_{2}+\ldots+a_{k-1}+a_{k}, \quad S_{2}(n)=b_{1}+b_{2}+\ldots+b_{k-1}+b_{n} .
$$

Notice that this specification is exactly the same as is required in the finite case. In fact, we know that when we have a sum with a finite number of addends it is not sufficient to say that the number, $l$, of addends in the sum is finite, it is necessary to assign explicitly a value to $l$ applying for this purpose numerals available in a traditional numeral system chosen to express finite numbers. For different finite values of $l$ the result of summation can be different. 
It is necessary to stress that (6.10) and (6.11) describe the same mathematical objects sums with an infinite number of addends - but with different accuracies. In the case (6.10), the numeral $\infty$ is used and the number of addends is not fixed - it is just said that it is infinite. The (1)-based lens allows us to observe that each divergent series is not a unique object with a generic infinite number of addends expressed by the numerals $\infty$. Records from (6.11) were visible like unique objects due to the low accuracy of the numeral $\infty$. Now we are able to distinguish different sums $S_{1}(k)$ and $S_{2}(n)$ in (6.11) and for different infinite values of $k$ and $n$ different results can be obtained. Analogously, Pirahã observing sums $1+1+1+1$ and $1+1+1+1+1$ are not able to distinguish two sums and see them as the same object having many addends whereas an observer able to count up to 5 will see two different sums.

Notice also that it would not be correct to say that, e.g., the record $S_{1}=\sum_{i=1}^{\infty} a_{i}$ from (6.10) represents a 'true infinite series that includes all possible addends $a_{i}$ ' whereas records (6.11) where the number of summands should be expressed by concrete infinite (1)-based numbers are some 'partial infinite sums'. The sentence 'all possible addends' means that the numeral $\infty$ being the upper index of summation represents the largest possible integer (if it is not the largest one then we do not consider all possible addends). Thus, the operation $\infty+1$ should be prohibited since we are not allowed to increase $\infty$ that represents the largest possible integer (notice that an interesting arithmetic where there exists the largest integer $K$ and the operation $K+1$ is not allowed is given in [75]) and the operation $\infty-1$ should give a result different from $\infty$ since in this case we have less addends in the sum. In other words, the assumption that $S_{1}=\sum_{i=1}^{\infty} a_{i}$ includes all possible addends $a_{i}$ leads by necessity to attribute to the symbol $\infty$ properties that are different from its traditional usage, where relations $\infty+1=\infty$ and $\infty-1=\infty$, etc. hold. Therefore, the meaning 'sum of all possible addends $a_{i}$ ' cannot be attributed to the record $S_{1}=\sum_{i=1}^{\infty} a_{i}$ if the numeral $\infty$ is used in its conventional sense. Thus, records (6.10) and (6.11) describe the same mathematical objects - sums with an infinite number of addends - but with different accuracies.

The following two comments should be made before we start to work with applications. First, notice that it is not possible to use (1)-based numerals in the same expression where the numeral $\infty$ is present since they belong to two different numeral systems having different accuracies. Such a record as $\infty+$ (1) has no meaning because $\infty$ is not defined within (1)-based numeral system and, vice versa, (1) is not defined in traditional mathematical languages using $\infty$. Analogously, the record 'many'+4 has no sense (Pirahã do not understand what 4 is and people understanding this are not able to assign a concrete value to 'many').

The second comment is required to link series to infinite sequences discussed in the previous section. Notice that even though a sequence cannot have more than (1) elements, the number of items in a series can be greater than grossone because the process of summing up is not necessary executed by a sequential adding of summands.

Let us show how the (1)-based approach can be applied to calculate sums where an infinite (1)-based number of summands has been specified.

Example 6.6. We consider two infinite series $S_{1}=7+7+7+\ldots$ and $S_{2}=3+3+3+\ldots$ As was already mentioned for a general case, traditional analysis gives us a very poor 
information about them and such operations as, e.g., $\frac{S_{2}}{S_{1}}$ and $S_{2}-S_{1}$ are not defined. In order to use the (1)-based approach, it is necessary to indicate explicitly the number of their summands.

Suppose that the sum $S_{1}$ has $k$ addends and $S_{2}$ has $n$ addends and, therefore, they are defined as follows

$$
S_{1}(k)=\underbrace{7+7+7+\ldots+7}_{k \text { addends }}, \quad S_{2}(n)=\underbrace{3+3+3+\ldots+3}_{n \text { addends }} .
$$

Then $S_{1}(k)=7 k$ and $S_{2}(n)=3 n$ and by assigning different numerical values (finite or infinite) to $k$ and $n$ we obtain different numerical values for the sums. Notice that the new numerals for the chosen $k$ and $n$ allow us to calculate $S_{2}(n)-S_{1}(k)$ (analogously, the expression $\frac{S_{1}(k)}{S_{2}(n)}$ can be calculated). If, for instance, $k=5(1)$ and $n=11$ we obtain $S_{1}\left(5(1)=35(1), S_{2}(1)=3(1)\right.$ and it follows

$$
S_{2}(1)-S_{1}(51)=3(1)-3511=-321<0 .
$$

If $k=3(1)$ and $n=711+2$ we obtain $S_{1}(31)=21(1), S_{2}(1)=21(1)+6$ and it follows

$$
\left.S_{2}(71)+2\right)-S_{1}(31)=21(1)+6-21(1)=6 .
$$

It is also possible to calculate sums having an infinite number of infinite or infinitesimal summands

$$
S_{3}(l)=\underbrace{211+2(1)+\ldots+21}_{l \text { addends }}, \quad S_{4}(m)=\underbrace{4(1)^{-1}+4\left(1^{-1}+\ldots+41^{-1}\right.}_{m \text { addends }} .
$$

For $l=m=0.5(1)$ it follows $S_{3}\left(0.5(1)=\left(1^{2}\right.\right.$ and $S_{4}\left(0.5(1)=2\left(\right.\right.$ recall that $(1) \cdot(1)^{-1}=(1)^{0}=$ 1 (see (4.18)). It can be seen from this example that it is possible to obtain finite numbers as the result of summing up infinitesimals. This is a direct consequence of Postulate 3.

The infinite and infinitesimal numbers allow us to calculate also arithmetic and geometric sums with an infinite number of items. Traditional approaches tell us that if $a_{n}=a_{1}+(n-1) d$ then for a finite $n$ it is possible to use the formula

$$
\sum_{i=1}^{n} a_{i}=\frac{n}{2}\left(a_{1}+a_{n}\right)
$$

In the (1)-based methodology we can use it for infinite values of $n$, as well.

Example 6.7. The sum of all natural numbers from 1 to (1) can be calculated as follows

$$
1+2+3+\ldots+(1)-1)+(1)=\sum_{i=1}^{1} i=\frac{1}{2}\left(1+(1)=0.51^{2}+0.51\right. \text {. }
$$

Let us calculate now the following sum of infinitesimals where each item is (1) times less than the corresponding item of (6.12)

$$
\left(1^{-1}+2(1)^{-1}+\ldots+(1)-1\right) \cdot(1)^{-1}+(1) \cdot(1)^{-1}=\sum_{i=1}^{(1)} i\left(1^{-1}=\frac{(1)}{2}\left(1^{-1}+1\right)=0.51^{1}+0.5\right. \text {. }
$$


Obviously, the obtained number, $0.51^{1}+0.5$ is (1) times less than the sum in (6.12). This example shows, in particular, that infinite numbers can also be obtained as the result of summing up infinitesimals.

Let us consider now a geometric series $\sum_{i=0}^{\infty} q^{i}$. Traditional analysis proves that it converges to $\frac{1}{1-q}$ for $q$ such that $-1<q<1$. We are able to give a more precise answer for all values of $q$. To do this we should fix the number of items in the sum. If we suppose that it contains $n$ items, then

$$
Q_{n}=\sum_{i=0}^{n} q^{i}=1+q+q^{2}+\ldots+q^{n} .
$$

By multiplying the left-hand and the right-hand parts of this equality by $q$ and by subtracting the result from (6.13) we obtain

$$
Q_{n}-q Q_{n}=1-q^{n+1}
$$

and, as a consequence, for all $q \neq 1$ the formula

$$
Q_{n}=\left(1-q^{n+1}\right)(1-q)^{-1}
$$

holds for finite and infinite $n$. Thus, the possibility to express infinite and infinitesimal numbers allows us to take into account infinite values of $n$ and the infinitesimal value $q^{n+1}$ for finite $q$. Moreover, we can calculate $Q_{n}$ for infinite and finite values of $n$ and $q=1$, because in this case we have just

$$
Q_{n}=\underbrace{1+1+1+\ldots+1}_{n+1 \text { addends }}=n+1 \text {. }
$$

Example 6.8. As the first example we consider the divergent series

$$
1+3+9+\ldots=\sum_{i=0}^{\infty} 3^{i}
$$

To determine it, we should specify the number $n$ of addends in it and, for example, for $n=(1)^{2}$ we obtain

$$
\sum_{i=0}^{(1)^{2}} 3^{i}=1+3+9+\ldots+3^{(1)^{2}}=\frac{1-3^{(1)^{2}+1}}{1-3}=0.5\left(3^{\left(1^{2}+1\right.}-1\right) .
$$

Analogously, for $n=(1)^{2}+1$ we obtain

$$
1+3+9+\ldots+3^{(1)^{2}}+3^{(1)^{2}+1}=0.5\left(3^{(1)^{2}+2}-1\right) .
$$

If we now find the difference between the two sums

$$
0.5\left(3^{(1)^{2}+2}-1\right)-\left(0.5\left(3^{(1)^{2}+1}-1\right)\right)=3^{(1)^{2}+1}(0.5 \cdot 3-0.5)=3^{(1)^{2}+1}
$$

we obtain the newly added item $3^{(1)^{2}+1}$. 
Example 6.9. In this example, we consider the series $\sum_{i=1}^{\infty} \frac{1}{2^{i}}$. It is well-known that it converges to one. However, we are able to give a more precise answer. In fact, due to Postulate 3, the formula

$$
\sum_{i=1}^{n} \frac{1}{2^{i}}=\frac{1}{2}\left(1+\frac{1}{2}+\frac{1}{2^{2}}+\ldots+\frac{1}{2^{n-1}}\right)=\frac{1}{2} \cdot \frac{1-\frac{1}{2}^{n}}{1-\frac{1}{2}}=1-\frac{1}{2^{n}}
$$

can be used directly for infinite $n$, too. For example, if $n=$ (1) then

$$
\sum_{i=1}^{(1)} \frac{1}{2^{i}}=1-\frac{1}{2^{\circledR}}
$$

where $\frac{1}{2^{\Phi}}$ is infinitesimal. Thus, the traditional answer $\sum_{i=1}^{\infty} \frac{1}{2^{i}}=1$ is just a finite approximation of our more precise result using infinitesimals.

6.3. Riemann series theorem and Ramanujan summation. Let us consider now series with alternating signs. Traditionally, convergence of series is studied and only for unconditionally convergent series rearrangements of addends do not affect the sum. The Riemann series theorem states that conditionally convergent series can be rearranged in such a way that they either diverge or converge to an arbitrary real number. In its turn, the (1)-based methodology shows that Riemann's result is a consequence of the weak numeral system used traditionally to work with infinite series. In fact, the usage of (1) allows us to see that (as it happens in the case where the number of addends is finite) rearrangements do not change the result for any sum with a fixed (finite or infinite) number of summands.

Example 6.10. Let us start from the famous series

$$
S_{5}=1-1+1-1+1-1+\ldots
$$

In the literature there exist many approaches giving different answers regarding the value of this series (see [60]). All of them use various notions of average (for instance, Cesàro summation assigns the value $\frac{1}{2}$ to $S_{5}$ ). However, sum and its average of any kind are different notions. In our approach we do not appeal to averages and calculate the required sum directly. To do this, we should indicate explicitly the number of items, $k$, in the sum. Then it follows that

$$
S_{5}(k)=\underbrace{1-1+1-1+1-1+1-\ldots}_{k \text { addends }}= \begin{cases}0, & \text { if } k=2 n, \\ 1, & \text { if } k=2 n+1,\end{cases}
$$

and it is not important whether $k$ is finite or infinite. For example, $S_{5}(1)=0$ because the number $\frac{1}{2}$ being the result of division of (1) by 2 has been introduced as the number of elements of a set and, therefore, it is integer. As a consequence, (1) is even. Analogously, $\left.S_{5}(1)-1\right)=1$ because $11-1$ is odd.

As it happens in the cases where the number of addends in a sum is finite, the result of summation does not depend on the way the summands are rearranged. In fact, if we 
know the exact infinite number of addends and the order the signs are alternated is clearly defined, we know also the exact number of positive and negative addends in the sum.

Let us illustrate this point by supposing, for instance, that we want to rearrange addends in the sum $S_{5}(21)$ in the following way

$$
S_{5}(21)=1+1-1+1+1-1+1+1-1+\ldots
$$

The traditional tools give an impression that this rearrangement modifies the result. However, in the (1)-based framework we know that this is just a consequence of the weak lens used to observe infinite numbers. In fact, thanks to (1) we are able to fix an infinite number of summands. In our example the sum has 21 addends, the number 21 is even and, therefore, it follows from $(6.15)$ that $S_{5}(21)=0$. This means also that in the sum there are (1) positive and (1) negative items. As a result, addition of the groups 1+1-1 considered above can continue only until the positive units present in the sum will not finish and then there will be necessary to continue to add only negative summands. More precisely, we have

$$
S_{5}(21)=\underbrace{1+1-1+1+1-1+\ldots+1+1-1}_{\text {(1) positive and } \frac{\mathbb{\Phi}}{2} \text { negative addends }} \underbrace{-1-1-\ldots-1-1-1}_{\frac{\Phi}{2} \text { negative addends }}=0 \text {, }
$$

where the result of the first part in this rearrangement is calculated as $(1+1-1) \cdot \frac{1}{2}=\frac{\Phi}{2}$ and the result of the second part summing up negative units is equal to $-\frac{\mathbb{D}}{2}$, thus yielding the same final result as (6.15). It becomes clear from (6.16) the origin of the Riemann series theorem. In fact, the second part of (6.16) containing only negative units is invisible if one works with the traditional numeral $\infty$.

Example 6.11. Let us consider now the following divergent series

$$
S_{6}=1-2+3-4+\ldots
$$

It can be easily considered as the difference of two arithmetic progressions after we have fixed the number of items, $k$, in the sum $S_{6}(k)$. Suppose that it contains grossone items. Then it follows

$$
\begin{gathered}
\left.\left.S_{6}(1)=1-2+3-4+\ldots-(1)-2\right)+(1-1)-1\right)= \\
(1+3+5+\ldots+(1-3)+(1-1))-(2+4+6+\ldots+(1-2)+(1)= \\
\frac{(1+(1-1)(1)}{4}-\frac{(2+1)(1)}{4}=\frac{(1)^{2}-21-(1)^{2}}{4}=-\frac{1}{2} .
\end{gathered}
$$

Notice that some traditional summation techniques can be interpreted as certain weighted averages on sums having (1) addends (see [114, 115] for a detailed discussion). Hereinafter we consider what happens in the (1)-based framework in situations where certain summation techniques lead to negative sums of divergent series that contain infinitely many positive integers. 
Example 6.12. Suppose that in the traditional fashion we have

$$
x=1+2+4+8+\ldots
$$

Let us proceed in the following way (that is similar to Ramanujan summation, to be considered in the next example). First we multiply both parts of (6.17) by 2 and obtain

$$
2 x=2+4+8+\ldots
$$

By adding 1 to both parts of (6.18) we obtain

$$
2 x+1=1+2+4+8+\ldots
$$

Thus, the right-hand side of (6.19) is equal to $x$ from (6.17) and after the substitution we get

$$
2 x+1=x, \quad x=-1
$$

and, as the final result, it follows

$$
1+2+4+8+\ldots=-1 .
$$

To define the value $x$ from (6.17) using the (1) methodology, it is necessary to indicate the number of addends in this infinite sum. Suppose that this is an infinite number $n$ and, therefore, $x$ should depend on $n$ and be defined as

$$
x(n)=1+2+4+8+\ldots+2^{n-1} .
$$

We proceed now in the manner illustrated above, i.e., we multiply both parts of this equality by 2 , add 1 to both left-hand and right-hand parts of (6.21), and obtain

$$
\begin{gathered}
2 x(n)+1=\underbrace{1+2+4+8+\ldots+2^{n-1}}_{x(n)}+2^{n}, \\
2 x(n)+1=x(n)+2^{n}, \quad x(n)=2^{n}-1 .
\end{gathered}
$$

Thus, for different infinite (and finite) values of $n$ we obtain different values of $x(n)$. Notice, that ' -1 ' that is visible using the traditional way of doing in (6.20) is visible in (6.23), as well. However, (6.23) contains also the term ' $2^{n}$ ' that was invisible before in spite of the fact that it is infinite for infinite values of $n$. Clearly, by assigning different values to $n$ we have different results in (6.23). For example, for $n=(1$ we have $x(1)=$ $2^{\Phi}-1$ and for $n=3\left(1\right.$ it follows that $x(31)=2^{3 \oplus}-1$. Let us mention that this result is consistent with the general formula (6.14); it is sufficient to take $q=2$ and the respective values of $n$ in (6.14).

The following example considers the famous result of Ramanujan

$$
c=1+2+3+4+5+\ldots=-\frac{1}{12}
$$

viewed through the lens of the grossone methodology (the symbol $c$ in (6.24) has been chosen since Ramanujan himself had used this notation). Remind that the sum of all 
natural numbers has been already calculated in the (1)-based framework in (6.12). The example below shows that by using Ramanujan's approach and paying attention to the infinite number of addends in sums involved in the consideration one arrives to the same conclusion as in (6.12).

Example 6.13. Let us first recall how Ramanujan derives (6.24). He multiplies the lefthand part of (6.24) by 4 and then subtracts the result from (6.24) as follows

$$
\begin{aligned}
& c=1+2+3+4+5+6+\ldots \\
& \begin{array}{l}
4 c=4 \\
3 c=1+8
\end{array}+12+\ldots
\end{aligned}
$$

Ramanujan then uses the result (considered in various forms by Euler, Cesàro and Hölder) attributing to the alternating series $1-2+3-4+\ldots$ the value $\frac{1}{4}$ as the formal power series expansion of the function $\frac{1}{(1+x)^{2}}$ for $x=1$, i.e.,

$$
1-2+3-4+\ldots=\frac{1}{4}
$$

Thus, it follows from (6.25) and (6.26) that

$$
-3 c=1-2+3-4+5-6+\ldots=\frac{1}{4}
$$

from where Ramanujan gets (6.24).

Let us use now the (1) lens and observe Ramanujan's procedure through it. First, it is necessary to indicate an infinite number of addends, $n$, in the sum

$$
c(n)=1+2+3+4+5+\ldots+n .
$$

The (1) methodology allows us to compute this sum for infinite values of $n$ directly (see (6.12)) and, therefore, multiplication by 4 leads to the following result

$$
4 c(n)=4+8+12+\ldots+4 n=4(1+2+3+\ldots+n)=4 \cdot \frac{n}{2}(1+n) .
$$

Then, subtraction gives us the final answer where the left-hand part calculated by (6.12) perfectly corresponds to the right-hand one

$$
-3 c(n)=-3-6-9-\ldots-3 n=-3(1+2+3+\ldots+n)=-3 \cdot \frac{n}{2}(1+n) .
$$

Notice also that Example 6.11 considers the series (6.26) providing the result valid for infinite values of $n$ and consistent with the respective result for its finite values

$$
1-2+3-4+\ldots+(n-1)-n=-\frac{n}{2}
$$

thus showing that (6.26) is just a consequence of the weakness of traditional tools used to study series. 
However, the main trick in (6.25) consists of displacement of addends in its second line. Without loss of generality let us see what happens with this shift in the case we have $n=(1)$ addends in $c(n)$. This means that both sums (6.27) and (6.28) have (1) summands each. However, the displacement of (6.25) proposes to sum up each number $i$ from the first line of (6.25) with the number $4 \cdot \frac{i}{2}$ for all even $i$, while all odd $i$ are summed up with zeros, i.e., they are just moved directly to the third line of (6.25). The important novelty offered by the (1) methodology is that now we know that since $n=1$ in our case we have $1 \leq i \leq 1$. This means that to $\frac{1}{2}$ even addends in $(6.27)$ there will be added the first $\frac{1}{2}$ numbers from (6.28), the last addend (1) from (6.27) corresponds to the number $4 \cdot \frac{1}{2}$ in (6.28). As a consequence, in (6.28) there will remain $\frac{1}{2}$ more addends, namely, they are

$$
4 \cdot\left(\frac{1}{2}+1\right)+4 \cdot\left(\frac{1}{2}+2\right)+\ldots+4 \cdot(1)
$$

The record (6.25) does not allow us to observe them whereas thanks to (1) these summands are perfectly visible and, therefore, (6.25) can be re-written using (6.27) - (6.30) in the following, more accurate than it is done traditionally, way

$$
\begin{array}{rlcc}
c(1) & = & 1+2+3+4+5+\ldots+(1)-1+(1) \\
4 c(1) & = & 4+8+\ldots \quad+4 \frac{1}{2}+4\left(\frac{1}{2}+1\right)+\ldots+41 \\
-3 c(1) & = & 1-2+3-4+5+\ldots+(1)-1-(1)-4\left(\frac{1}{2}+1\right)-\ldots-41
\end{array}
$$

Let us compute now the right-hand part of the third line of (6.31) by using Example 6.11 for the first (1) addends and the arithmetical progression formula for the remaining $\frac{1}{2}$ summands

$$
\begin{aligned}
1-2+3-4 & +5+\ldots+(1)-1-(1)-4\left(\frac{1}{2}+1\right)-4\left(\frac{1}{2}+2\right)-\ldots-41= \\
& -\frac{1}{2}-4\left(\left(\frac{1}{2}+1\right)+\left(\frac{1}{2}+2\right)+\ldots+(1)\right)= \\
& \left.-\frac{1}{2}-4 \cdot \frac{(1)}{2}\left(\left(\frac{1}{2}+1\right)+(1)\right)=-3 \frac{1}{2}(1)+1\right) .
\end{aligned}
$$

As was expected, the left-hand part of the third line of (6.31) computed using (6.29) for $n=(1)$ provides the same result.

6.4. From limits to expressions evaluated at actual infinite/infinitesimal points. As is well known, historically infinitesimals played a pivotal role in the differential calculus (see $[63,77]$ ). However, at that time they lacked a precise mathematical definition and were gradually replaced by the d'Alembert-Cauchy concept of limit (see $[19,28])$ that we use nowadays. Thus, actual infinities and infinitesimals have been substituted by potential ones (e.g., in $\lim _{x \rightarrow a} f(x)$ we do not evaluate $f(\infty)$ we work with $x$ tending to $\infty$ ). The modern rigor has been given to Leibniz's program in non-standard analysis (see 
[82]), however, due to its symbolic character, this approach does not allow one to evaluate expressions in actual infinite and infinitesimal points, it works with generic infinite and infinitesimal variables only. The problem of assigning concrete values to these variables is not considered in non-standard analysis.

The (1)-based methodology allows us to evaluate expressions at different actual infinite and infinitesimal points in the same way as we do it with finite constants. It becomes possible to substitute limits by precise infinite and infinitesimal numbers that can be written explicitly and to calculate expressions even when limits do not exist. Thus, $\lim _{x \rightarrow b} f(x)=\infty$ can be substituted by precise infinite numbers that can be different in dependence on the value of $x$. In case $b=\infty$ one should choose a concrete infinite (1)-based number $x$ where to evaluate $f(x)$, in case $b=0$, the chosen $x$ should be infinitesimal, and in case $b$ is finite, $x$ should have a finite part and an infinitesimal one (positive or negative). Analogously, $\lim _{x \rightarrow b} f(x)=0$ can be substituted by precise infinitesimal numbers that can be different in dependence on the value of $x$. Finally, in case there exists a finite value $a$ such that $\lim _{x \rightarrow b} f(x)=a$, in the (1)-based framework $a$ is just a finite approximation of a number $A=a+\alpha$ where $\alpha$ is an infinitesimal expressed in (1)-based numerals.

The following examples give some illustrations. The first one compares results provided by traditional analysis, non-standard analysis, and the (1)-based approach.

Example 6.14. If $x$ is a fixed finite number then

$$
\lim _{h \rightarrow 0} \frac{(x+h)^{2}-x^{2}}{h}=2 x
$$

In a non-standard analysis framework it follwos

$$
\frac{(x+h)^{2}-x^{2}}{h}=2 x+h
$$

where $h$ is a generic infinitesimal. In its turn, the (1)-based approach allows us to work with different values of $h$. If, for instance, $h=(1)^{-1}$, the answer is $2 x 1^{0}(1)^{-1}$, if $h=4.21^{-2}$ we obtain the value $2 x(1)^{0} 4.2(1)^{-2}$, etc. Thus, the value of the limit (6.32), for a finite $x$, is just the finite approximation of the number (6.33) having the finite part equal to $2 x$ and infinitesimal parts can vary in dependence on the value of $h$.

Example 6.15. Let us consider the following two limits

$$
\lim _{x \rightarrow+\infty}\left(5 x^{3}-x^{2}+10^{61}\right)=+\infty, \quad \lim _{x \rightarrow+\infty}\left(5 x^{3}-x^{2}\right)=+\infty .
$$

Both give us the same result, $+\infty$, and it is not possible to execute numerically the operation

$$
\lim _{x \rightarrow+\infty}\left(5 x^{3}-x^{2}+10^{61}\right)-\lim _{x \rightarrow+\infty}\left(5 x^{3}-x^{2}\right) .
$$

that is an indeterminate form of the type $\infty-\infty$ in spite of the fact that for any finite $x$ it follows

$$
5 x^{3}-x^{2}+10^{61}-\left(5 x^{3}-x^{2}\right)=10^{61}
$$


The new approach allows us to calculate exact values of both expressions, $5 x^{3}-x^{2}+10^{61}$ and $5 x^{3}-x^{2}+10$, at any infinite (and infinitesimal) $x$ expressible in the chosen numeral system. For instance, the choice $x=3(1)^{2}$ gives the value

$$
\left.5(31)^{2}\right)^{3}-\left(3(1)^{2}\right)^{2}+10^{61}=135(1)^{6}-911^{4} 10^{61}
$$

for the first expression and $1351^{6}-91^{4}$ for the second one. We can easily calculate the difference of these two infinite numbers, thus obtaining the same result as we had for finite values of $x$ in (6.34):

$$
1351^{6}-9(1)^{4} 10^{61}-\left(1351^{6}-91^{4}\right)=10^{61} .
$$

Notice that the possibility of the direct evaluation of expressions is very important (in particular, for automatic computations) because it eliminates indeterminate forms from the practice of computations. For instance, in the traditional language if for a finite $a$, $\lim _{x \rightarrow a} f(x)=0$ and $\lim _{y \rightarrow \infty} g(y)=\infty$ then $\lim _{x \rightarrow a} f(x) \cdot \lim _{y \rightarrow \infty} g(y)$ is an indeterminate form. In the (1)-based framework, this means that for any $x=a+z$ where $z$ is infinitesimal, the value $f(a+z)$ is also infinitesimal and for any infinite $y$ it follows that $g(y)$ is also infinite. In order to be able to execute computations, we should behave ourselves as we are used to do in the finite case. Namely, it is necessary to choose values of $z$ and $y$ and to evaluate $f(a+z)$ and $g(y)$. After we have performed these operations it becomes possible to execute multiplication $f(a+z) \cdot g(y)$ and to obtain the corresponding result that can be infinite, finite or infinitesimal in dependence on the values of $z$ and $y$ and the form of expressions $f(x)$ and $g(y)$.

It is possible also to execute other operations with infinitesimals and infinities making questions with respect to $f(a+z)$ and $g(y)$ that could not even be formulated using the traditional language using limits. For instance, we can ask about the result of the following expression

$$
f\left(a+z_{2}\right)\left(\frac{g\left(y_{1}\right)}{f\left(a+z_{1}\right)}-1.25 g\left(y_{2}\right)^{3}\right)
$$

for two different infinitesimals $z_{1}, z_{2}$ and two different infinite values $y_{1}$ and $y_{2}$.

Example 6.16. Let us consider computation of the product $f(a+z) \cdot g(y)$. For the sake of simplicity we take $a=0, g(y)=y$, and

$$
f(x)= \begin{cases}2 x, & x<0 \\ 1, & x=0 \\ x^{3}, & x>0\end{cases}
$$

If we want to calculate the product at points $z=1^{-1}$ and $y=(1)$ then it follows

$$
f(a+z) \cdot g(y)=f\left(\mathbb{1}^{-1}\right) \cdot g(\mathbb{1})=\mathbb{1}^{-3} \cdot(1)=\mathbb{1}^{-2} .
$$

Analogously, $z=1^{-1}$ and $y=\left(1^{4}\right.$ yield

$$
f\left(1^{-1}\right) \cdot g\left(1^{4}\right)=\left(1 ^ { - 3 } \cdot \left(1^{4}=(1)^{1}\right.\right.
$$


and for $z=-21^{-1}$ and $y=(1)$ we obtain

$$
f\left(-21^{-1}\right) \cdot g(1)=-41^{-1} \cdot(1)=-4 .
$$

We end this example by calculating the result of the expression (6.35) for $z_{1}=-21^{-1}$, $z_{2}=-51^{-4}, y_{1}=(1)^{2}$, and $y_{2}=(1)$. These values provide

$$
\begin{aligned}
& f\left(a+z_{2}\right)\left(\frac{g\left(y_{1}\right)}{f\left(a+z_{1}\right)}-1.25 g\left(y_{2}\right)^{3}\right)=f\left(-51^{-4}\right)\left(\frac{\left.g(1)^{2}\right)}{f\left(-21^{-1}\right)}-1.25 g(1)^{3}\right)= \\
& \left.-101)^{-4} \cdot\left(\frac{1^{2}}{-41^{-1}}-1.25(1)^{3}\right)=-101\right)^{-4} \cdot\left(-0.25(1)^{3}-1.25(1)^{3}\right)=15(1)^{-1}
\end{aligned}
$$

It is worthwhile to mention that expressions can be calculated even when their limits do not exist. Thus, we obtain a very powerful tool for studying divergent processes.

Example 6.17. The following $\lim _{n \rightarrow+\infty} f(n), f(n)=(-1)^{n} n^{3}$, does not exist. However, we can easily calculate expression $(-1)^{n} n^{3}$ at different infinite points $n$. For instance, for $n=$ (1) it follows $f(1)=1^{3}$ because grossone is even and for the odd $n=0.511-1$ it follows

$$
f(0.5(1)-1)=-(0.5(1)-1)^{3}=-0.125(1)^{3}+0.75(1)^{2}-1.5(1)^{1}+1 .
$$

Let us make a remark regarding irrational numbers. Among their properties, they are characterized by the fact that we do not know any numeral system that would allow us to express them by a finite number of symbols used to express other numbers. Thus, special numerals $(e, \pi, \sqrt{2}, \sqrt{3}$, etc.) are introduced by describing their properties in some way. These special symbols are then used in analytical transformations together with ordinary numerals.

For example, it is possible to work directly with the symbol $e$ in analytical transformations by applying suitable rules defining this number together with numerals taking part in a chosen numeral system $\mathcal{S}$. At the end of transformations, the obtained result will be be expressed in numerals from $\mathcal{S}$ and, probably, in terms of $e$. If it is then required to execute some numerical computations, this means that it is necessary to substitute $e$ by a numeral (or numerals) from $\mathcal{S}$ that will allow us to approximate $e$ in some way.

The same situation takes place when one uses the (1)-based numeral system, i.e., while we work analytically we use just the symbol $e$ in our expressions and then, if we wish to work numerically we should move to approximations. The (1)-based numeral system opens a new perspective on the problem of the expression of irrational numbers. Let us consider one of the possible ways to obtain an approximation of $e$, i.e., by using the limit

$$
e=\lim _{n \rightarrow+\infty}\left(1+\frac{1}{n}\right)^{n}=2.71828182845904 \ldots
$$

In our numeral system the expression $\left(1+\frac{1}{n}\right)^{n}$ can be written directly for finite and/or infinite values of $n$. For $n=$ (1) we obtain the number $e_{0}$, so designated in order to distinguish it from the record (6.36)

$$
e_{0}=\left(1+1^{-1}\right)^{\circledR} \text {. }
$$


It becomes clear from this record why the number $e$ cannot be expressed in a positional numeral system with a finite base. Due to the definition of a sequence under the IUA, such a system can have at maximum (1) numerals - digits - to express fractional part of a number (see sections 6.1, 7.3 for details) and, as it can be seen from (6.37), this quantity is not sufficient for $e$ because the item $\frac{1}{\left(1^{1}\right.}$ is present in it.

Naturally, it is also possible to construct more exotic $e$-type numbers by substituting $n$ in the expression $\left(1+\frac{1}{n}\right)^{n}$ with any infinite number written in the (1)-based positional system. For example, if $n=\left(1^{2}\right.$ we obtain the number $e_{1}=\left(1+(1)^{-2}\right)^{\Phi^{2}}$. The numbers considered above take their origins in the limit (6.36). The same way of reasoning can be used with respect to other irrational numbers, as well.

6.5. Functions, continuity, derivatives, and integrals. The goal of this subsection is to discuss mathematical and physical definitions of continuity and to develop a new, Physicsdriven point of view on this notion using the (1)-based infinities and infinitesimals. The new point of view is illustrated by a detailed consideration of one of the most fundamental mathematical definitions - function. Then, the notion of continuity is studied in depth and computations of derivatives and integrals are illustrated.

As was already mentioned in Introduction (see discussion on page 3 ) in modern Physics the continuity of an object is relative. For example, if we observe a table by eye, then we see it continuous. If we use a microscope for our observation, we see that the table is discrete. This means that we decide how to regard the object, as a continuous or as a discrete one, by choosing a suitable instrument of observation.

In traditional Mathematics any mathematical object is either continuous or discrete (e.g., the same function cannot be both continuous and discrete). This contraposition of discrete and continuous in traditional Mathematics does not reflect properly the modern physical interpretations showing that the continuity of an object $A$ depends on the accuracy of the instrument used for the observation of $A$. The (1)-based methodology gives us a possibility to develop a theory of continuity that is closer to the physical world and better reflects the new discoveries made by physicists. We start by introducing a definition of the one-dimensional continuous set of points based on the above considerations and Postulate 2 and establish relations to such a fundamental notion as function using infinities and infinitesimals.

Let us remind that traditionally a function $f(x)$ is defined as a binary relation among two sets $X$ and $Y$ (called the domain and the codomain of the relation) with the additional property that to each element $x \in X$ corresponds exactly one element $f(x) \in Y$. We consider now a function $f(x)$ defined over a one-dimensional interval $[a, b]$. It follows from the previous sections that to define a function $f(x)$ over an interval $[a, b]$ it is not sufficient to give a rule for evaluating $f(x)$ and the values $a$ and $b$ because we are not able to evaluate $f(x)$ at any point $x \in[a, b]$. Only points $x$ expressible in known numeral systems can be used in computations. However, the traditional definition of function does not pay attention to the problem of expressibility of points from the domain at which $f(x)$ can be evaluated. This fact in certain cases can lead to ambiguity.

Therefore, in order to be precise in the definition of a function, it is necessary to indicate explicitly a numeral system, $\mathcal{S}$, we intend to use to express points from the interval 
$[a, b]$. Thus, a function $f(x)$ is defined when we know a rule allowing us to obtain $f(x)$ given $x$ and its domain, i.e., the set $[a, b]_{\mathcal{S}}$ of points $x \in[a, b]$ expressible in the chosen numeral system $\mathcal{S}$. We suppose hereinafter that the system $\mathcal{S}$ is used to write down $f(x)$ (of course, the choice of $\mathcal{S}$ determines a class of formulae and/or procedures we are able to express using $\mathcal{S}$ ) and it allows us to express any number

$$
y=f(x), \quad x \in[a, b]_{\mathcal{S}} .
$$

The number of points of the domain $[a, b]_{\mathcal{S}}$ can be finite or infinite but the set $[a, b]_{\mathcal{S}}$ is always discrete. This means that for any point $x \in[a, b]_{\mathcal{S}}$ it is possible to determine its closest right and left neighbors, $x^{+}$and $x^{-}$, respectively, as follows

$$
x^{+}=\min \left\{z: z \in[a, b]_{\mathcal{S}}, \quad z>x\right\}, \quad x^{-}=\max \left\{z: z \in[a, b]_{\mathcal{S}}, \quad z<x\right\} .
$$

Apparently, the obtained discrete construction leads us to the necessity to abandon the nice idea of continuity, which is a very useful notion used in different fields of Mathematics. But this is not the case. In contrast, the new approach allows us to introduce a new definition of continuity very well reflecting the physical world.

Let us consider $n+1$ points

$$
a=x_{0}<x_{1}<x_{2}<\ldots<x_{n-1}<x_{n}=b
$$

and suppose that we have a numeral system $S$ allowing us to calculate their coordinates using a unit of measure $\mu$ (for example, meter, inch, etc.) and to construct the set $X=$ $[a, b]_{\mathcal{S}}$ expressing these points.

The set $X$ is called continuous in the unit of measure $\mu$ if for any $x \in(a, b)_{\mathcal{S}}$ it follows that the differences $x^{+}-x$ and $x-x^{-}$from (6.38) expressed in units $\mu$ are equal to infinitesimal numbers. In our numeral system with grossone this means that all the differences $x^{+}-x$ and $x-x^{-}$contain only negative (finite or infinite) grosspowers. Note that it becomes possible to differentiate types of continuity by taking into account values of grosspowers of infinitesimal numbers (continuity of order $1^{-1}$, continuity of order $\left(^{-2}\right.$, etc.).

This definition emphasizes the physical principle that there does not exist an absolute notion of continuity: it is relative with respect to the chosen instrument of observation which in our case is represented by the unit of measure $\mu$. Thus, the same set can be viewed as continuous or discrete in dependence of the chosen unit of measure.

Example 6.18. Suppose that the set of six equidistant points

$$
X_{1}=\left\{a, x_{1}, x_{2}, x_{3}, x_{4}, x_{5}\right\}
$$

from Fig. 6.1 has the distance $d$ between the points equal to $1^{-1}$ in a unit of measure $\mu$ and, therefore, it is continuous in $\mu$. Usage of a new unit of measure $v=(1)^{-3} \mu$ implies that $d=(1)^{2}$ in $v$ and the set $X_{1}$ is not continuous in $v$.

Note that the introduced definition does not require that all the points from $X$ are equidistant. For instance, if in Fig. 6.1 for a unit measure $\mu$ the largest distance $x_{6}-x_{5}$ over the set $[a, b]_{\mathcal{S}}$ is infinitesimal then the whole set is continuous in $\mu$. 


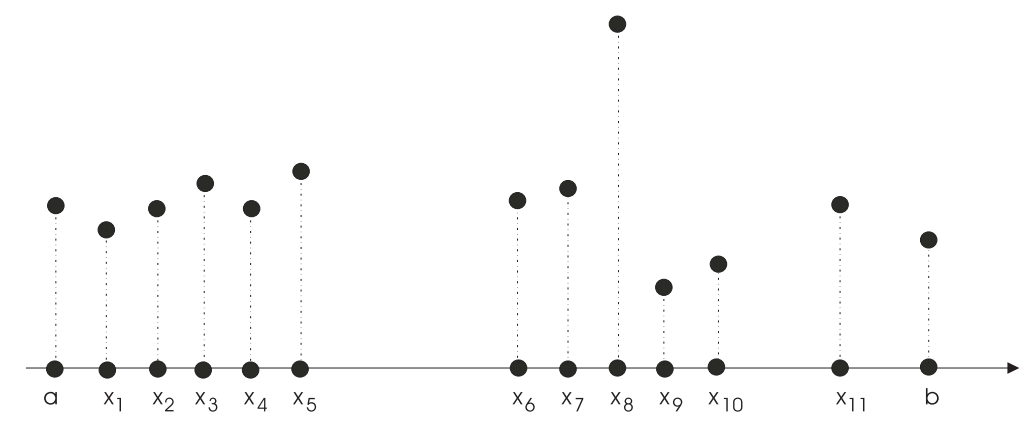

Figure 6.1. It is not possible to say whether this function is continuous or discrete until we have not introduced a unit of measure and a numeral system to express distances between the points

The set $X$ is called discrete in the unit of measure $\mu$ if for all points $x \in(a, b)_{\mathcal{S}}$ it follows that the differences $x^{+}-x$ and $x-x^{-}$from (6.38) expressed in units $\mu$ are not infinitesimal numbers. In our numeral system with radix grossone this means that in all the differences $x^{+}-x$ and $x-x^{-}$negative grosspowers cannot be the largest ones. For instance, the set $X_{1}$ from (6.40) is discrete in the unit of measure $v$ from Example 6.18. Of course, it is also possible to consider intermediate cases where sets have continuous and discrete parts.

The introduced notions allow us to give the following very simple definition of a function continuous at a point. A function $f(x)$ defined over a set $[a, b]_{\mathcal{S}}$ continuous in a unit of measure $\mu$ is called continuous in the unit of measure $\mu$ at a point $x \in(a, b)_{\mathcal{S}}$ if both differences $f(x)-f\left(x^{+}\right)$and $f(x)-f\left(x^{-}\right)$are infinitesimal numbers in $\mu$, where $x^{+}$and $x^{-}$are from (6.38). For continuity at points $a, b$ it is sufficient that one of these differences is infinitesimal. The notions of continuity from the left and from the right in a unit of measure $\mu$ at a point are introduced naturally. Similarly, the notions of a function discrete, discrete from the right, and discrete from the left can be defined.

The function $f(x)$ is continuous in the unit of measure $\mu$ over the set $[a, b]_{\mathcal{S}}$ if it is continuous in $\mu$ at all points of $[a, b]_{\mathcal{S}}$. Again, it becomes possible to distinguish types of continuity by taking into account values of grosspowers of infinitesimal numbers (continuity of order $(1)^{-1}$, continuity of order $(1)^{-2}$, etc.) and to consider functions in such units of measure that they become continuous or discrete over certain subintervals of $[a, b]$. In what follows, we shall often fix the unit of measure $\mu$ and write just 'continuous function' instead of 'continuous function in the unit of measure $\mu$ '. Let us give three simple examples illustrating the introduced definitions.

Example 6.19. We start by showing that the function $f(x)=x^{2}$ is continuous over the set $X_{2}$ defined as the interval $[0,1]$ where numerals $\frac{i}{1}, 0 \leq i \leq 1$, are used to express its points in units $\mu$. First of all, note that the set $X_{2}$ is continuous in $\mu$ because its points are equidistant with the distance $d=1^{-1}$. Since this function is strictly increasing, to show its continuity it is sufficient to check the difference $f(x)-f\left(x^{-}\right)$at the point $x=1$. In this 
case, $x^{-}=1-(1)^{-1}$ and we have

$$
f(1)-f\left(1-1^{-1}\right)=1-\left(1-1^{-1}\right)^{2}=21^{-1}-1^{-2} .
$$

This number is infinitesimal, thus $f(x)=x^{2}$ is continuous over the set $X_{2}$.

Example 6.20. Consider the same function $f(x)=x^{2}$ over the set $X_{3}$ defined as the interval [1 -1 , (1)] where numerals (1) $-1+\frac{i}{1}, 0 \leq i \leq 1$, are used to express its points in units $\mu$. Analogously, the set $X_{3}$ is continuous and it is sufficient to check the difference $f(x)-f\left(x^{-}\right)$at the point $x=$ (1) to show continuity of $f(x)$ over this set. In this case,

$$
\begin{gathered}
x^{-}=\left(1-1+\frac{1}{1}-1\right. \\
(1)-(1)^{-1}, \\
\left.\left.f(x)-f\left(x^{-}\right)=f(1)-f(1)-(1)^{-1}\right)=(1)^{2}-(1)-(1)^{-1}\right)^{2}=2-(1)^{-2} .
\end{gathered}
$$

This number is not infinitesimal because it contains the finite part 2 and, as a consequence, $f(x)=x^{2}$ is not continuous over the set $X_{3}$.

Example 6.21. Consider $f(x)=x^{2}$ defined over the set $X_{4}$ being the interval [1 - 1, (1) where numerals (1) $-1+\frac{i}{(1)^{2}}, 0 \leq i \leq(1)^{2}$, are used to express its points in units $\mu$. The set $X_{4}$ is continuous and we check the difference $f(x)-f\left(x^{-}\right)$at the point $x=1$. We have

$$
\begin{gathered}
x^{-}=(1)-1+\frac{1^{2}-1}{1^{2}}=(1-1)^{-2}, \\
\left.f(x)-f\left(x^{-}\right)=f(1)-f(1)-(1)^{-2}\right)=(1)^{2}-\left(1-(1)^{-2}\right)^{2}=21^{-1}(-1)(1)^{-4} .
\end{gathered}
$$

Since the obtained result is infinitesimal, $f(x)=x^{2}$ is continuous over $X_{4}$.

Let us consider now a function $f(x)$ defined by formulae over a set $X=[a, b]_{\mathcal{S}}$ so that different expressions can be used over different subintervals of $[a, b]$. The notion 'formula' hereinafter indicates a single expression used to evaluate $f(x)$.

Example 6.22. The function $g(x)=2 x^{2}-1, x \in[a, b]_{\mathcal{S}}$, is defined by one formula and

$$
f(x)= \begin{cases}\max \left\{-10 x, 5 x^{-1}\right\}, & x \in[c, 0)_{\mathcal{S}} \cup(0, d]_{\mathcal{S}}, \quad c<0, \quad d>0, \\ 4 x, & x=0,\end{cases}
$$

is defined by three formulae, $f_{1}(x), f_{2}(x)$, and $f_{3}(x)$ where

$$
\begin{array}{ll}
f_{1}(x)=-10 x, & x \in[c, 0)_{\mathcal{S}}, \\
f_{2}(x)=4 x, & x=0, \\
f_{3}(x)=5 x^{-1}, & x \in(0, d]_{\mathcal{S}},
\end{array}
$$

i.e., in the neighborhood of the point $x=0$ three different expressions are used to evaluate $f(x)$. 
In general, a function $f(x)$ is defined in a neighborhood of a point $x$ as follows

$$
f(\xi)= \begin{cases}f_{1}(\xi), & x-l \leq \xi<x, \\ f_{2}(\xi), & \xi=x, \\ f_{3}(\xi), & x<\xi \leq x+r,\end{cases}
$$

where the number $l$ is any number such that the same formula $f_{1}(\xi)$ is used to define $f(\xi)$ at all points $\xi$ such that $x-l \leq \xi<x$. Analogously, the number $r$ is any number such that the same formula $f_{3}(\xi)$ is used to define $f(\xi)$ at all points $\xi$ such that $x<\xi \leq x+r$. Of course, as a particular case it is possible that the same formula is used to define $f(\xi)$ over the interval $[x-l, x+r]$, i.e.,

$$
f(\xi)=f_{1}(\xi)=f_{2}(\xi)=f_{3}(\xi), \quad \xi \in[x-l, x+r] .
$$

It is also possible that (6.44) does not hold but formulae $f_{1}(\xi)$ and $f_{3}(\xi)$ are defined at the point $x$ and are such that at this point they return the same value, i.e.,

$$
f_{1}(x)=f_{2}(x)=f_{3}(x)
$$

If condition (6.45) holds, we say that function $f(x)$ has continuous formulae at the point $x$. Of course, in the general case, formulae $f_{1}(\xi), f_{2}(\xi)$, and $f_{3}(\xi)$ can be or cannot be defined out of the respective intervals from (6.43). In cases where condition (6.45) is not satisfied we say that function $f(x)$ has discontinuous formulae at the point $x$. Definitions of functions having formulae which are continuous or discontinuous from the left and from the right are introduced naturally.

Example 6.23. Let us study the following function

$$
f(x)= \begin{cases}1^{2}+\frac{x^{2}-1}{x-1}, & x \neq 1, \\ a, & x=1,\end{cases}
$$

at the point $x=1$. By using designations (6.43) and the fact that for $x \neq 1$ it follows $\frac{x^{2}-1}{x-1}=x+1$ we have

$$
f(\xi)= \begin{cases}f_{1}(\xi)=(1)^{2}+\xi+1, & \xi<1, \\ f_{2}(\xi)=a, & \xi=1, \\ \left.f_{3}(\xi)=1\right)^{2}+\xi+1, & \xi>1,\end{cases}
$$

Since

$$
f_{1}(1)=f_{3}(1)=1^{2}+2, \quad f_{2}(1)=a,
$$

we obtain that if $a=(1)^{2}+2$, then the function (6.46) has continuous formulae ${ }^{11}$ at the point $x=1$. Analogously, the function (6.41) has continuous formulae at the point $x=0$ from the left and discontinuous from the right.

\footnotetext{
${ }^{11}$ Note, that even if $a=(1)^{2}+2+\varepsilon$, where $\varepsilon$ is an infinitesimal number expressed in (1)-based numerals (recall that all infinitesimals are different from zero), we are able to establish that the function has discontinuous formulae.
} 
Thus, functions having continuous formulae at a point can be continuous or discrete at this point in dependence of the chosen unit of measure. Analogously, functions having discontinuous formulae at a point can be continuous or discrete at this point again in dependence of the chosen unit of measure. The notion of continuity of a function depends on the chosen unit of measure and numeral system $S$ and it can be used for functions defined by formulae, computer procedures, tables, etc. In contrast, the notion of a function having continuous formulae works only for functions defined by formulae and does not depend on units of measure or numeral systems chosen to express its domain. It is related only to properties of formulae.

The present numerical point of view on the definition of continuity has been extended in [91] to the differential calculus for one-dimensional functions assuming finite, infinite, and infinitesimal values over finite, infinite, and infinitesimal domains. Let us give a few illustrations.

Example 6.24. Let us consider the function $f(x)=x^{2}$ and its first derivative $f^{\prime}(x)=$ $2 x$. Thanks to (1)-based numerals both $f(x)$ and $f^{\prime}(x)$ can be evaluated at infinite and infinitesimal points. For instance, for the infinite $x=$ (1) we obtain infinite values $f(1)=$ (1) ${ }^{2}$ and $f^{\prime}(1)=2(1)$. For the infinitesimal $x=(1)^{-1}$ we have infinitesimal values $f\left(1^{-1}\right)=$ (1) ${ }^{-2}$ and $f^{\prime}\left(1^{-1}\right)=21^{-1}$.

We can also work with functions having formulae including infinite and infinitesimal numbers. For example, the function $f(x)=\frac{1}{1} x^{2}+(1) x$ has the first derivative $f^{\prime}(x)=$ $\frac{2}{1} x+$ (1). For the infinite $x=$ (1) we obtain infinite values

$$
f(1)=(1)+(1)^{2}, \quad f^{\prime}(1)=2+\text { (1) }
$$

For the infinitesimal $x=\left(1^{-1}\right.$ we have the value $f\left(1^{-1}\right)=1+(1)^{-3}$ having a finite and an infinitesimal part and the first derivative is infinite having also an infinitesimal part, namely, it follows $f^{\prime}\left(1^{-1}\right)=(1)+21^{-2}$.

Analogously to the passage from series to sums with a fixed infinite number of items, the introduction of infinite and infinitesimal numerals allows us (in fact, it imposes) to substitute improper integrals of various kinds by integrals defined in a more precise way. For example, let us consider the following improper integral

$$
\int_{0}^{\infty} x^{2} d x
$$

Now it is necessary to define its upper infinite limit of integration explicitly. Then, different infinite numbers used instead of $\infty$ will lead to different results, as it happens in the finite case where different finite limits of integration lead to different results.

Example 6.25. For instance, numbers (1) and $\left(1^{2}\right.$ give us two different integrals both assuming infinite but different values

$$
\int_{0}^{(1)} x^{2} d x=\frac{1}{3}{ }^{(1)^{3}}, \quad \int_{0}^{(1)^{2}} x^{2} d x=\frac{1}{3}{ }^{1}{ }^{6} .
$$


Moreover, it becomes possible to calculate integrals where both endpoints of the interval of integration are infinite as in the following example

$$
\int_{(1)}^{(1)^{2}} x^{2} d x=\frac{1}{3}(1)^{6}-\frac{1}{3}(1)^{3}
$$

It becomes possible to calculate integrals of functions assuming infinite and infinitesimal values and the results of integration can be finite, infinite, and infinitesimal. For instance, in the integral

$$
\int_{(1)}^{(1)+(1)^{-2}} x^{2} d x=\frac{1}{3}\left(\left(1^{1}+(1)^{-2}\right)^{3}-\frac{1}{3}\left(1^{3}=11^{0}+11^{-3}+\frac{1}{3}(1)^{-6}\right.\right.
$$

the result has a finite part and two infinitesimal parts and in the integral

$$
\int_{(1)}^{(1)+(1)^{-2}} x^{2}-x d x=11^{-3}-\frac{1}{2}(1)^{-4}+\frac{1}{3}\left(1^{-6}\right.
$$

the result has three infinitesimal parts. The last two examples illustrate situations when the integrand is infinite

$$
\int_{(1)}^{(1)^{2}}(1) x^{2} d x=(1) \int_{(1)}^{(1)^{2}} x^{2} d x=\frac{1}{3}(1)^{7}-\frac{1}{3}(1)^{4}
$$

and infinitesimal

$$
\int_{(1)}^{(1)^{2}}{ }^{-4} x^{2} d x=(1)^{-4} \int_{(1)}^{(1)^{2}} x^{2} d x=\frac{1}{3}\left(1^{2}-\frac{1}{3}{ }^{(1-1} .\right.
$$

\section{Measuring infinite sets and the Continuum Hypothesis}

7.1. Measuring infinite sets with elements defined by formulae. We have already discussed how to calculate the number of elements of sets resulting from the usual set theoretical operations (intersection, union, etc.) with finite sets and infinite sets of the type $\mathbb{N}_{k, n}$ from (4.2). In order to work with infinite sets having a more general structure than the sets $\mathbb{N}_{k, n}$, we need to develop more powerful instruments. Suppose that we have an integer function $g(i)>0$ strictly increasing on indexes $i=1,2,3, \ldots$ and we wish to know how many elements there are in the set

$$
G=\{g(1), g(2), g(3), \ldots\} .
$$

In the (1)-based terminology this question is meaningless because of the following reason.

In the finite case, to define a set it is not sufficient just to say that it is finite. It is necessary to indicate its number of elements explicitly as, e.g., in this example

$$
G_{1}=\{g(i): 1 \leq i \leq 5\}
$$


or implicitly, by saying that elements satisfying a certain condition belong to the set, for instance, as it is done below

$$
G_{2}=\{g(i): i \geq 1,0<f(i) \leq b\}
$$

where $b$ is finite.

Thanks to the (1)-based numeral system we have mathematical tools to express the number of elements for infinite sets, too. Thus, analogously to the finite case and due to Postulate 3 , it is not sufficient to say that a set has infinitely many elements. It is necessary to indicate its number of elements explicitly or implicitly. For instance, the number of elements of the set

$$
G_{3}=\left\{g(i): 1 \leq i \leq \mathbb{1}^{10}\right\}
$$

is indicated explicitly: the set $G_{3}$ has ${ }^{10}{ }^{10}$ elements.

If a set is given in the form (7.1) where $b$ is infinite, then its number of elements, $J$, can be determined as

$$
J=\max \{i: g(i) \leq b\}
$$

if we are able to determine the inverse function $g^{-1}(x)$ for $g(x)$. Then, $J=\left\lfloor g^{-1}(b)\right\rfloor$, where $\lfloor u\rfloor$ is integer part of $u$. Notice that if $b=(1)$, then the set $G_{2} \subseteq \mathbb{N}$ since all its elements are integer, positive, and $g(i) \leq 1$ due to (7.2).

Example 7.1. Let us consider the following set, $A_{1}(k, n)$, having $g(i)=k+n(i-1)$,

$$
A_{1}(k, n)=\{g(i): i \geq 1, g(i) \leq(1)\}, \quad 1 \leq k \leq n, \quad n \in \mathbb{N}
$$

It follows from the IUA that $A_{1}(k, n)=\mathbb{N}_{k, n}$ from (4.2). By applying (7.2) we find for $A_{1}(k, n)$ its number of elements

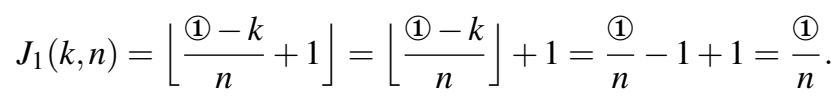

Analogously, the set

$$
\left.A_{2}(k, n, j)=\left\{k+n i^{j}: i \geq 0,0<k+n i^{j} \leq 1\right)\right\}, \quad 0 \leq k<n, \quad n \in \mathbb{N}, \quad j \in \mathbb{N},
$$

has

$$
J_{2}(k, n, j)=\left\lfloor\sqrt[j]{\frac{1-k}{n}}\right\rfloor
$$

elements. In particular, this means that the set, $I^{2}$ of square natural numbers traditionally written as

$$
I^{2}=\left\{x: x \in \mathbb{N}, i \in \mathbb{N}, x=i^{2}\right\}=\{1,4,9,16,25, \ldots\}
$$

is $A_{2}(0,1,2)$ and, therefore, it has $\lfloor\sqrt{(1)}\rfloor$ elements. Notice that the same result has been obtained in [66] using alternative instruments. 
As was already discussed in section 4.2, the new approach does not contradict the oneto-one correspondence principle. The traditional record (famously mentioned by Galileo Galilei) establishes bijection among the sets $I^{2}$ and $\mathbb{N}$ as follows

$$
\begin{array}{ccccccc}
1, & 2^{2}, & 3^{2}, & 4^{2}, & 5^{2}, & 6^{2}, & \ldots \\
\downarrow & \downarrow & \uparrow & \uparrow & \downarrow & \downarrow & \\
1, & 2, & 3, & 4 & 5, & 6, & \ldots
\end{array}
$$

but does not allow one to see that these two sets have different numbers of elements. The answer that both sets are countable is correct but its accuracy is low. The (1)-based methodology allows us to see the difference in their number of elements and to express the final part of (7.4), as follows

$$
\begin{array}{ccccccccc}
1, & 2^{2}, & 3^{2}, & 4^{2}, & 5^{2}, & \ldots & \left(\left\lfloor 1^{1 / 2}\right\rfloor-2\right)^{2}, & \left(\left\lfloor 1^{1 / 2}\right\rfloor-1\right)^{2}, & \left\lfloor 1^{1 / 2}\right\rfloor^{2} \\
\uparrow & \uparrow & \uparrow & \uparrow & \uparrow & & \uparrow & \uparrow & \uparrow \\
1, & 2, & 3, & 4 & 5, & \ldots & \left\lfloor(1)^{1 / 2}\right\rfloor-2, & \left.\lfloor 1)^{1 / 2}\right\rfloor-1, & \left.\lfloor 1)^{1 / 2}\right\rfloor
\end{array}
$$

Infinite sets defined by other functions $g(i)$ from (7.2) can be studied by a complete analogy.

7.2. Countable sets can be measured more accurately. Remind that Postulate 2 stresses the difference between mathematical objects and the tools used to observe them. In this section, infinite sets of numbers are our objects of observation and numeral systems are our instruments. If we fix a numeral system $\mathcal{S}$ and a set $A$ then the following three situations can take place with respect to expressibility of the number of elements, $J$, of the set $A$ in $S$ : (i) $\mathcal{S}$ is able to express $J$ exactly, i.e., to the accuracy of one element; (ii) $S$ is able to express $J$ with a low accuracy (Pirahã would say that the set of 5 apples has many apples; this answer is correct but its accuracy is low); (iii) $\mathcal{S}$ is not able to express $J$ at all. The last option can occur for several reasons. For instance, because $J$ cannot be determined (e.g., the inequality from (7.2) cannot be solved or the formula for elements of the set is not known). Another possibility is where the set $A$ consists of numbers not expressible in $\mathcal{S}$ (or $A$ contains among its elements some numbers of this kind) and, therefore, $A$ itself cannot be observed through $\mathcal{S}$. Finally, a numeral that is required to express $J$ may not belong to $S$. Thus, each numeral system can express exactly the number of elements only of a limited number of sets. Let us consider some sets having countable or continuum cardinalities and see what the (1)-based numeral system can say with respect to the number of elements of these sets. The designation $|A|$ will be used hereinafter to indicate the number of elements of a set $A$ expressed in (1)-based numerals.

We have already seen above that the set, $\mathbb{N}$, of natural numbers has (1) elements, the set, $\mathbb{Z}$, of integers has $2(1+1$ elements (see example 4.7 ), and the sets, $\mathbb{E}$ and $\mathbb{O}$, of even and odd numbers have $\frac{1}{2}$ elements each. Let us study the set of rational numbers now. Traditionally, rational numbers are defined as ratios of two integers. The new approach allows us to calculate the number of numerals in a fixed numeral system. Let us consider a numeral system $\mathbb{Q}_{1}$ containing numerals of the form

$$
\frac{p}{q}, \quad p \in \mathbb{Z}, \quad q \in \mathbb{Z}, q \neq 0 .
$$


Theorem 7.2. The number of elements of the set, $\mathbb{Q}_{1}$, of rational numerals of the type (7.6) is $\left|\mathbb{Q}_{1}\right|=41^{2}+21$.

Proof. Since the set $\mathbb{Z}$ has $21+1$ elements, the numerator of (7.6) can be filled in by $21+$ 1 and the denominator by 21 numbers. Thus, the number of all possible combinations is

$$
(21)+1) \cdot 2(1=41)^{2}+2(1)
$$

that gives us the value of $\left|\mathbb{Q}_{1}\right|$.

Notice that we have calculated the quantity of different rational numerals of the form (7.6) and not the quantity of different rational numbers. For example, in the numeral system $\mathbb{Q}_{1}$ the number 0 can be expressed by 21 different numerals

$$
\frac{0}{-1}, \frac{0}{-(1+1}, \frac{0}{-(1)+2}, \ldots \frac{0}{-2}, \frac{0}{-1}, \frac{0}{1}, \frac{0}{2}, \ldots \frac{0}{(1)-2}, \frac{0}{(1)-1}, \frac{0}{1}
$$

and numerals such as $\frac{-1}{-2}, \frac{1}{2}$, and $\frac{2}{4}$ representing the same number have been calculated as three different numerals. The following theorem determines the number of elements of the set $\mathbb{Q}_{2}$ containing numerals of the form

$$
-\frac{p}{q}, \frac{p}{q}, \quad p \in \mathbb{N}, \quad q \in \mathbb{N},
$$

where zero is represented by the symbol 0 .

Theorem 7.3. The number of elements of the set, $\mathbb{Q}_{2}$, of rational numerals of the type (7.7) is $\left|\mathbb{Q}_{2}\right|=21^{2}+1$.

Proof. Let us consider positive rational numerals. The form of the rational numeral $\frac{p}{q}$, the fact that $p, q \in \mathbb{N}$, and the IUA impose that both $p$ and $q$ can assume values from 1 to 1 . Thus, the number of all possible combinations is $\left(1^{2}\right.$. The same number of combinations is obtained for negative rational numbers and one is added because we count zero as well.

Notice that the introduction of grossone allows us to observe rationals that were not visible through traditional numeral systems used to express finite numbers. In fact, both kinds of rational numerals, (7.6) and (7.7), allow us to see rationals that are infinities, infinitesimals or finite but contain infinitesimal parts. For instance, the following numbers can be represented by (7.6) and (7.7)

$$
\frac{-5}{(1)}, \frac{2}{(1)-6}, \frac{1-1}{(1)-2}=1+\frac{1}{(1)-2}, \frac{1-2}{(1)-1}=1-\frac{1}{(1)-1}, \frac{1-5}{3}, \frac{1}{2} .
$$

The first two of them are infinitesimals, the next two numbers are finite with infinitesimal parts, and the last two numbers are infinite. In the same way as we have introduced sets, $\widehat{\mathbb{N}}$ and $\mathbb{Z}$, of extended natural and integer numbers, it is possible to consider the set, $\widehat{\mathbb{Q}}$, of extended rational numbers such that in the fraction $\frac{p}{q}$ numbers $p$ and $q$ can be integers larger than (1), for instance, the number $\frac{3}{5(1)}$ is not rational since its denominator is larger 
Table 7.1. Cardinalities and the number of elements of some infinite sets.

\begin{tabular}{|c|c|c|}
\hline $\begin{array}{c}\text { Description } \\
\text { of sets }\end{array}$ & $\begin{array}{c}\text { Cantor's } \\
\text { cardinalities }\end{array}$ & $\begin{array}{l}\text { Number of } \\
\text { elements }\end{array}$ \\
\hline the set of natural numbers $\mathbb{N}$ & countable, $\boldsymbol{\aleph}_{0}$ & (1) \\
\hline $\mathbb{N} \backslash\{3,5,10,23,114\}$ & countable, $\boldsymbol{\aleph}_{0}$ & (1)-5 \\
\hline the set of even numbers $\mathbb{E}$ (the set of odd numbers $(\mathbb{O})$ & countable, $\boldsymbol{\aleph}_{0}$ & $\frac{(1)}{2}$ \\
\hline the set of integers $\mathbb{Z}$ & countable, $\boldsymbol{\aleph}_{0}$ & $2(1)+1$ \\
\hline $\mathbb{Z} \backslash\{0\}$ & countable, $\boldsymbol{\aleph}_{0}$ & 2(1) \\
\hline squares of natural numbers $\mathbb{G}=\left\{x: x=n^{2}, x \in \mathbb{N}, n \in \mathbb{N}\right\}$ & countable, $\boldsymbol{\aleph}_{0}$ & $\lfloor\sqrt{1}\rfloor$ \\
\hline pairs of natural numbers $\mathbb{P}=\{(p, q): p \in \mathbb{N}, q \in \mathbb{N}\}$ & countable, $\aleph_{0}$ & $(1)^{2}$ \\
\hline the set of numerals $\mathbb{Q}_{1}=\left\{\frac{p}{q}: p \in \mathbb{Z}, q \in \mathbb{Z}, q \neq 0\right\}$ from (7.6) & countable, $\boldsymbol{\aleph}_{0}$ & $4(1)^{2}+21$ \\
\hline the set of numerals $\mathbb{Q}_{2}=\left\{0,-\frac{p}{q}, \frac{p}{q}: p \in \mathbb{N}, q \in \mathbb{N}\right\}$ from (7.7) & countable, $\boldsymbol{\aleph}_{0}$ & $2(1)^{2}+1$ \\
\hline the power set of the set of natural numbers $\mathbb{N}$ & continuum, $\mathrm{c}$ & $2^{(1)}$ \\
\hline the power set of the set of even numbers $\mathbb{E}$ & continuum, $\mathrm{c}$ & $2^{0.5(1)}$ \\
\hline the power set of the set of integers $\mathbb{Z}$ & continuum, $\mathrm{c}$ & $2^{2(1)+1}$ \\
\hline the power set of the set of numerals $\mathbb{Q}_{1}$ from (7.6) & continuum, $\mathrm{c}$ & $2^{\left.4(1)^{2}+21\right)}$ \\
\hline the power set of the set of numerals $\mathbb{Q}_{2}$ from (7.7) & continuum, c & $2^{2(1)^{2}+1}$ \\
\hline numbers $x \in[0,1)$ expressible in the binary numeral system & continuum, $\mathrm{c}$ & $2^{(1)}$ \\
\hline numbers $x \in[0,1]$ expressible in the binary numeral system & continuum, $\mathrm{c}$ & $2^{(1)}+1$ \\
\hline numbers $x \in(0,1)$ expressible in the decimal numeral system & continuum, $\mathrm{c}$ & $10^{(1)}-1$ \\
\hline numbers $x \in[0,2)$ expressible in the decimal numeral s & continuum, $\mathrm{c}$ & $2 \cdot 10^{1}$ \\
\hline
\end{tabular}

than grossone but it belongs to $\widehat{\mathbb{Q}}$. As it was with the sets $\widehat{\mathbb{N}}$ and $\widehat{\mathbb{Z}}$, the (1)-based numerals do not allow us to express the number of elements of $\widehat{\mathbb{Q}}$.

Table 7.1 presents a comparison between the numeral system of Cantor and (1)-based numerals. It can be seen that the latter allow us to measure infinite sets more accurately instead of just saying that all these sets are countable. Moreover, Table 7.1 shows that the more accurate counting of the number of elements in countable sets leads to more accurate results (easily following from combinatorial arguments) for the number of elements of their power sets traditionally having the cardinality of continuum. Table 7.1 shows that the (1)-based numerals allow us to give more precise answers for these sets, as well. The next subsection is dedicated to other sets having the cardinality of the continuum (the number of elements for some of them are shown in the lower part of Table 7.1). 
7.3. The number of elements of sets having the cardinality of the continuum. Let us now consider real numbers and again pay attention to the separation of the object of observation from the tool used to observe the object. Traditionally in Mathematics there is no such separation and real numbers often are identified with numbers expressible in a positional numeral system. In the (1) framework, the real line is an abstract mathematical object that is observed by numeral systems and different numeral systems (that are our instruments of observation) allow us to see different sets of real numbers and the positional numeral system is just one of the possible lenses that can be used to observe real numbers. There exist also other lenses. As was already mentioned, the Roman numeral system does not allow us to see negative numbers and the Pirahã system only detects numbers 1 and 2 across the whole real line. So, once we have fixed a numeral system that we intend to use to express real numbers, we have decided which real numbers we can observe. The accuracy of the chosen lens fixes what can be observed and what cannot and, therefore, it does not make sense to talk about things that are not observable with the chosen lens. Instead of this, if we need a higher accuracy, it is better to change the present lens with a stronger one.

Let us consider the real numbers expressible in the positional numeral system, $\mathbb{R}_{b n q}$, with the finite integer radix $b$, where $n \in \mathbb{N}$ digits are used to express the integer part, and $q \in \mathbb{N}$ digits to write down the fractional part of a number, i.e., $\mathbb{R}_{b n q}$ uses the following numerals

$$
\left(a_{n-1} a_{n-2} \ldots a_{1} a_{0} \cdot a_{1} a_{2} \ldots a_{q-1} a_{q}\right)_{b} .
$$

The first observation that can be made concerns the number of numerals expressible in the form (7.8).

Theorem 7.4. The maximal number of numerals that can be expressed by the numeral system $\mathbb{R}_{\text {bnq }}$ is $\left|\mathbb{R}_{b(1) 1}\right|=b^{2(1)}$.

Proof. In formula (7.8), defining the type of numerals we deal with, there are two sequences of digits: the first one, $a_{n-1} a_{n-2} \ldots a_{1} a_{0}$, is used to express the integer part of the number and the second, $a_{1} a_{2} \ldots a_{q-1} a_{q}$, for representing its fractional part. Due to the definition of sequence and the IUA, each of them can have at maximum (1) elements. Thus, it can be at maximum (1) positions on the left of the dot in (7.8) and, analogously, (1) positions on the right of the dot. Every position can be filled in by one of the $b$ digits from the alphabet $\{0,1, \ldots, b-1\}$. Thus, we have $b^{(1)}$ combinations to express the integer part of the number and the same quantity to express its fractional part. As a result, the positional numeral system using the numerals of the form (7.8) can express $b^{2(1)}$ numbers.

Notice that all the numerals ${ }^{12}$ of the type (7.8) represent different numbers, the least and greatest numbers expressible in $\mathbb{R}_{b n q}$ can be explicitly indicated as well as the smallest positive number expressible in this system.

\footnotetext{
${ }^{12}$ The result of theorem 7.4 does not consider the practical situation of writing down concrete numerals. Obviously, the number of numerals of the type (7.8) that can be written down in practice is finite and depends on the chosen numeral system for writing digits. Theorem 7.4 shows us how many numerals of the type (7.8) exist.
} 
Example 7.5. For instance, in the decimal positional system $\mathbb{R}_{10,1,1}$ the numerals

$$
1 . \underbrace{999 \ldots 99}_{\text {(1) digits }}, \quad 2 . \underbrace{000 \ldots 00}_{(1) \text { digits }}
$$

represent different numbers and their difference is equal to the smallest positive number expressible in this system

$$
2 . \underbrace{000 \ldots 00}_{\text {(1) digits }}-1 . \underbrace{999 \ldots 9}_{\text {(1) digits }}=0 . \underbrace{000 \ldots 01}_{\text {(1) digits }} \text {. }
$$

Recall that the traditional point of view on real numbers implies that there exist real numbers that can be represented in positional systems by two different infinite sequences of digits, for instance, in the decimal positional system the records $2.000000 \ldots$ and $1.99999 \ldots$ represent the same number. As always, there is no contradiction between the traditional and the new points of view. They just use different lenses in their mathematical microscopes to observe numbers. The instruments used on the traditional view for this purpose are just too weak to distinguish two different numbers in the records $2.000000 \ldots$ and $1.99999 . .$.

Analogously the smallest and the largest numbers expressible in $\mathbb{R}_{b n q}$ can be easily indicated. For the numeral system $\mathbb{R}_{10111}$ they are, respectively,

$$
-\underbrace{999 \ldots 9}_{\text {(1) digits }} \cdot \underbrace{999 \ldots 9}_{\text {(1) digits }}, \quad \underbrace{999 \ldots 9}_{\text {(1) digits }} \cdot \underbrace{999 \ldots 9}_{\text {(1) digits }} .
$$

Recall that traditionally it is supposed that any positional numeral system is able to represent all real numbers ('the whole real line') and very often the real line is identified with numbers written in the positional numeral system. In the (1)-based framework we emphasize that any numeral system is just an instrument that can be used to observe certain real numbers. This instrument can be more or less powerful, e.g., the positional system (7.8) with the base $b=10$ can express $10^{2(1)}$ numerals and, therefore, it is more powerful than the positional system (7.8) with the radix $b=2$ that can express $2^{2(1)}<10^{211}$ numerals. Traditionally, this difference is invisible.

In general, two numeral systems can allow us to observe either the same sets of numbers, or sets of numbers having an intersection, or two disjoint sets of numbers. Due to Postulate 2, we are not able to answer the question 'What is the whole real line?' because this is the question asking 'What is the object of the observation?', we are able just to invent more and more powerful numeral systems that will allow us to improve our observations of numbers by using newly introduced numerals. Thus, questions that can be formulated and answered require first to fix a numeral system $\mathcal{S}$ (our lens of observation) and then to ask 'Which real numbers are expressible through $\mathcal{S}$ ?'.

The following theorem stresses once again the difference existing between objects of the observation (e.g., the field of real numbers) and what is viewed through our instruments (e.g., numerals expressible in $\mathbb{R}_{b n q}$ ).

Theorem 7.6. The sets $\mathbb{Z}, \mathbb{Q}_{1}, \mathbb{Q}_{2}$, and $\mathbb{R}_{\text {bnq }}$ are not monoids under addition.

Proof. The proof is obvious and thus omitted. 
7.4. Relations to results of Georg Cantor. In this subsection we revisit in the (1)-based framework some of results of Cantor related to measuring infinite sets. We start by calculating the number of points in the interval $[0,1)$ used in Cantor's diagonal argument to show that this number is uncountable. To do this we need a definition of the notion 'point' and mathematical tools that can be used to indicate a point. Since this concept is one of the most fundamental, it is very difficult to find an adequate definition for it (remind that in the (1)-based methodology it is better to say 'description' since any definition is done using a certain mathematical language $\mathcal{L}$ and, therefore, it allows us to say about the object only things expressible in $\mathcal{L}$, i.e., each definition describes the object with the accuracy of $\mathcal{L}$ ). If we accept (as is usually done in modern Mathematics) that a point in $[0,1)$ is determined by its coordinate $x$, then $x$ can be used both to indicate the point and to execute some computations with $x$ (for instance, to evaluate a function $f(u)$ at the point $u=x$ ). Since we can express coordinates only by numerals, different choices of numeral systems lead to various sets of numerals that can be used for expressing coordinates and, as a consequence, to different sets of points we can refer to. In each concrete case, the choice of a numeral system $\mathcal{S}$ will define what the word point means for us in this case and we shall not be able to work with the points whose coordinates are not expressible in the chosen numeral system $\mathcal{S}$. As a consequence, we are able to calculate the number of points over $[0,1)$ if we have already decided which numerals will be used to express the coordinates of points.

Different numeral systems can be chosen to express coordinates of points in dependence on the precision level we want to obtain and once this choice has been done it becomes possible to count the number of numerals available to express coordinates. For example, Pirahã are not able to express any point over the interval $[0,1)$. If numbers $x \in[0,1)$ are expressed in the form $\frac{p-1}{1}, p \in \mathbb{N}$, then the smallest positive number we can distinguish is $\frac{1}{1}$ and the interval $[0,1)$ contains the following (1) points

$$
0, \frac{1}{1}, \frac{2}{1}, \ldots \frac{(1)-2}{(1)}, \frac{(1)-1}{(1)} \text {. }
$$

If we want to count the number of intervals of the form $[a-1, a), a \in \mathbb{N}$, on the ray $x \geq 0$, then, by Postulate 3, the definition of sequence, and Theorem 6.1, not more than (1) intervals of this type can be distinguished on the ray $x \geq 0$. They are

$$
[0,1),[1,2),[2,3), \ldots[(1)-3,(1)-2),[(1)-2,(1)-1),[(1)-1,(1) .
$$

Within each of them we are able to distinguish (1) points of the kind $a+y$, where $y$ is from (7.9), and, therefore, across the entire ray (1) ${ }^{2}$ points of this type can be observed. Analogously, the ray $x<0$ is represented by the intervals

$$
[-(1),-(1)+1),[-(1)+1,-(1)+2), \ldots[-2,-1),[-1,0) .
$$

Hence, this ray also contains $\left(1^{2}\right.$ such points and on the whole line $2\left(1^{2}\right.$ points of this type can be represented and observed.

Note that the point - (1) is included in this representation and the point (1) is excluded from it. Let us slightly modify our numeral system in order to have (1) representable. For 
this purpose, intervals of the type $(a-1, a], a \in \mathbb{N}$, should be considered to represent the ray $x>0$ and the separate symbol, 0 , should be used to represent zero. Then, on the ray $x>0$ we are able to observe $\left(1^{2}\right.$ points and, analogously, on the ray $x<0$ we also are able to observe $1^{2}$ points. Finally, by adding the symbol used to represent zero we obtain that on the entire line $21^{2}+1$ points can be observed.

As was already mentioned, different numeral systems allow us to see the interval $[0,1)$ and the entire real line in different way. For instance, if only integers are used for this purpose, then over $[0,1)$ only the point $x=0$ is visible and the real line is represented by 2 (1) +1 points

$$
-(1),-1+1,-(1)+2, \ldots-2,-1,0,1,2, \ldots \text { (1) - 2, (1) - 1, (1). }
$$

In order to start a comparison with Cantor's results showing that the number of points of $[0,1)$ is uncountable, let us recall that he used the following numerals in his famous diagonal argument

$$
\left(. a_{1} a_{2} a_{3} a_{4} a_{5} a_{6} \ldots\right)_{b}
$$

belonging to a positional numeral system with a radix $b$. Notice that traditionally in (7.10) $b=2$ or $b=10$ are used and it is supposed that both values can express all real numbers over the interval $[0,1)$. Clearly, traditional numeral systems did not allow Cantor to see the end part of the sequence of digits (7.10). The (1)-based framework offers this possibility and the following positional numeral system $\mathbb{R}_{b 0 q}$

$$
\left(. a_{1} a_{2} \ldots a_{q-1} a_{q}\right)_{b}, \quad q \in \mathbb{N}
$$

being a particular case of the numeral system from (7.8) can be used for this purpose.

Theorem 7.7. The number of numerals of the type (7.11) for a fixed infinite value of $q$ is equal to $b^{q}$.

Proof. The prof follows immediately from Theorem 7.4.

Corollary 7.8. The maximal number of numerals of the type (7.11) is equal to $b^{(1)}$.

Proof. The proof is obvious and is thus omitted.

Corollary 7.9. The entire real line contains $21 b^{(1)}$ points of the type $a+y$, where $a$ is integer and $y$ is from (7.11) with $q=$ (1).

Proof. We have already seen above that it is possible to distinguish 21 unit intervals within the real line. Thus, the whole number of points of the type (7.11) on the line is equal to $21 b^{(1)}$.

In Corollary 7.9, we represented the real line by numerals (7.11) used in each of the intervals $[a, a+1)$ where $a \in \mathbb{Z}$. If we are not interested in subdividing the line at intervals and want to obtain the number of the points on the line directly by using positional numerals of the type (7.8) then, as it has already been established in Theorem 7.4, the number of points expressible by the numerals (7.8) is $\left|\mathbb{R}_{b}\right|=b^{2(1)}$. 
It is of the essence to stress that the results presented above can be considered as a more precise analysis of the situation discovered by Cantor. He has proved, by using his famous diagonal argument, that the number of elements of the set $\mathbb{N}$ is less than the number of real numbers at the interval $[0,1)$ without calculating the latter. To do this he expressed real numbers in a positional numeral system (7.10). We have shown that this number will be different depending on the radix $b$ used in the positional system to express real numbers. However, the resulting number, $b^{\circledR}$, of points expressible in this numeral system will be larger than the number of elements of the set of natural numbers, (1), for any radix $b>1$ and, therefore, the diagonal argument maintains its force.

The analysis made above shows that, as it was for countable sets, the (1)-based methodology allows us to realise that sets having the cardinality of continuum can have different infinite numbers of elements expressible in (1)-based numerals (see Table 7.1). Both numeral systems observe the same objects - infinite sets. However, Cantor through his lens distinguishes only two dots (countable and continuous) and the Continuum Hypothesis (metaphorically speaking) asks whether it is possible to distinguish other dots between them. The (1) lens instead of just two dots allows us to observe many different dots (i.e., infinite sets with different infinite numbers of elements). Moreover, it becomes possible to observe sets invisible to Cantor's lens. Some of them have a special importance with respect to the Continuum Hypothesis. They are studied in the following example.

Example 7.10. Let us consider again numerals (7.11). Due to construction, it is expected that for infinite values of $q$ the set $\mathbb{R}_{b 0 q}$ should have the cardinality of the continuum in the traditional language. Let us consider now $q_{1}=\left\lfloor\log _{b}(1\rfloor\right.$ where $\lfloor x\rfloor$, as usual, is the integer part ${ }^{13}$ of $x$. Notice that $q_{1}$ is infinite since (1) is infinite and $b$ is finite. It follows then that the number of numerals in $\mathbb{R}_{b 0 q_{1}}$ is

$$
b^{\left\lfloor\log _{b^{\boxplus}}\right\rfloor}<b^{\log _{b^{\oplus}}}=1 \text {, }
$$

i.e., with respect to the traditional language the set $\mathbb{R}_{b 0 q_{1}}$ would be countable. Analogously, many different instances of infinite sets that are constructed starting from the continuum framework and resulting at the end to be countable can be exhibited. For example, for infinite $q_{2}=3\left\lfloor\log _{b}(1)\right.$ and $q_{3}=0.5\left\lfloor\log _{b}(1\rfloor\right.$ it follows that

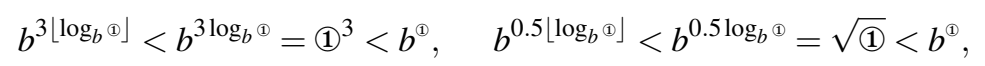

i.e., the sets of numerals $\mathbb{R}_{b 0 q_{2}}$ and $\mathbb{R}_{b 0 q_{3}}$ would be also countable from the traditional point of view.

Thus, thanks to the (1)-based numerals it becomes possible to calculate the exact number of elements of old (see Table 7.1) and new sets and to exhibit sets that were constructed as continuum but are indeed countable bridging so the gap between the two groups of sets. These results show that the difficulty of the Continuum Hypothesis is a consequence of the weakness of the numeral systems of Cantor's cardinals and the passage to (1)-based numerals allows one to avoid troubles. Analogously, it is difficult (impossible) to answer

\footnotetext{
${ }^{13}$ Notice that $\log _{b}$ (1) is not integer. It can be shown by the following simple argument. Suppose that there exist integers $b$ and $x$ such that $b^{x}=(1)$ and $b$ is a finite number. Then (1) would be divisible only by $b$ and this is impossible due to the IUA.
} 


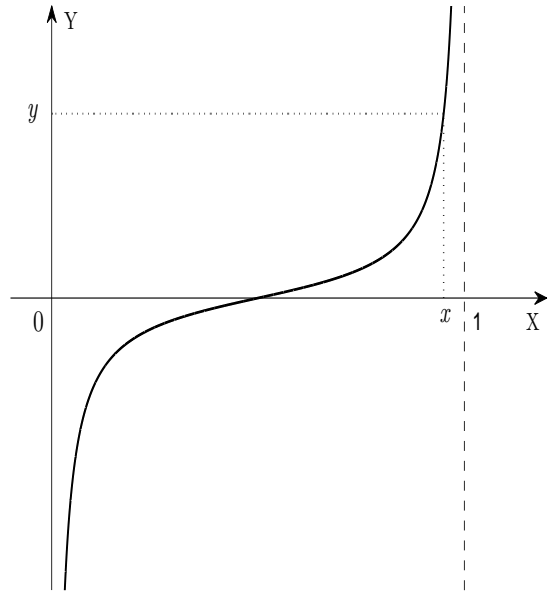

(a)

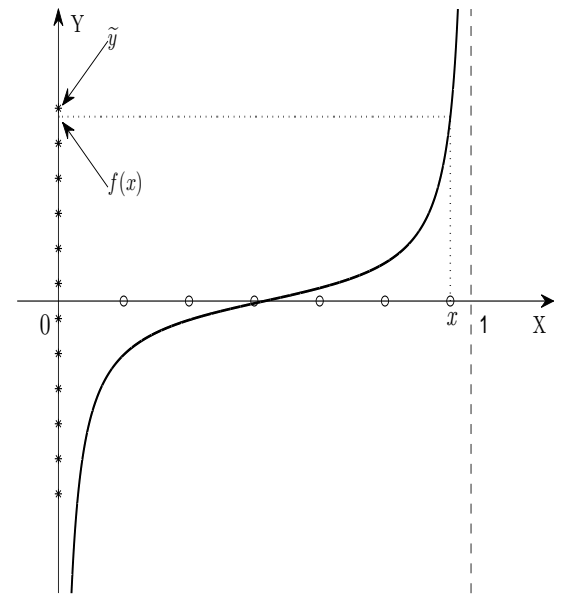

(b)

Figure 7.1. (a) Due to Cantor, the interval $(0,1)$ and the entire real line $Y$ have the same number of points. (b) The (1)-based framework allows us to observe three independent mathematical objects: the set $X_{\mathcal{S}_{1}}$ represented by small circles, the set $Y_{\mathcal{S}_{2}}$ represented by stars, and function (7.12)

to the question about the result of $\mathrm{V}-\mathrm{V}$ in Roman numerals that do not allow one to express zero whereas this problem vanishes if one works in a positional numeral system where zero can be expressed.

Clearly, the choice of a mathematical language depends on the practical problem that one intends to solve and on the accuracy required for such a solution. Such results as

$$
\mathfrak{\aleph}_{0}+1=\aleph_{0}, \quad \mathfrak{c}+\mathfrak{\aleph}_{0}=\mathfrak{c}, \quad \text { 'many'+1='many' }
$$

are correct. If one is satisfied with their accuracy, these answers can be successfully used in practice (even 'many' is used by Pirahã nowadays). However, if one needs more precise results, it is necessary to introduce a more powerful mathematical language (a numeral system in this case) allowing one to express the required answer in a more accurate way as the (1)-based numeral system does.

We conclude this section stressing once again that there is no contradiction between Cantor's results and the (1)-based framework. Bijections among countable sets have been already studied in section 4.2. Let us now return to the problem of comparison of infinite sets and consider Cantor's result showing that the cardinality of the set of points over the interval $(0,1)$ is equal to the cardinality of the set of points over the whole real line.

The proof of this counterintuitive fact is given by establishing a one-to-one correspondence between the elements of the two sets. Due to Cantor, such a mapping may be given, for example, by the function

$$
y=\tan (0.5 \pi(2 x-1)), \quad x \in(0,1),
$$

illustrated in Fig. 7.1(a). Cantor shows by using this figure that to any point $x \in(0,1)$ a point $y \in(-\infty, \infty)$ can be associated and vice versa. Thus, he concludes that the requested 
bijection between the sets $\mathbb{R}$ and $(0,1)$ has been established and, therefore, they have the same cardinality.

In the (1)-based methodology the number of elements of a set is its intrinsic characteristic (for both finite and infinite sets) that does not depend on any object outside the set. Thus, in Cantor's example from Fig. 7.1(a) we have (see Fig. 7.1(b)) three mathematical objects: (i) a set, $X_{\mathcal{S}_{1}}$, of points over the interval $(0,1)$ which we are able to distinguish using a fixed numeral system $S_{1}$; (ii) a set, $Y_{\mathcal{S}_{2}}$, of points over the vertical real line which we are able to distinguish using another fixed numeral system $\mathcal{S}_{2}$; (iii) the function (7.12) described using the third fixed numeral system $S_{3}$. All these three mathematical objects are independent of each other. The sets $X_{\mathcal{S}_{1}}$ and $Y_{\mathcal{S}_{2}}$ can have the same or a different number of elements.

It should be stressed that we are not able to evaluate $f(x)$ at any point $x$. We are able to do this only at points from $X_{\mathcal{S}_{1}}$ (even if we consider all numeral systems known to humanity, the number of points which we can express will be limited). Of course, in order to be able to execute these evaluations it is necessary to conciliate the numeral systems $\mathcal{S}_{1}, \mathcal{S}_{2}$, and $\mathcal{S}_{3}$. The fact that we have made evaluations of $f(x)$ and have obtained the corresponding values does not at all influence the numbers of elements of the sets $X_{\mathcal{S}_{1}}$ and $Y_{\mathcal{S}_{2}}$. Moreover, it can happen that the number $y=f(x)$ cannot be expressed in the numeral system $S_{2}$ and it is necessary to approximate it by a number $\tilde{y} \in \mathcal{S}_{2}$. This situation, very well known to computer scientists, is represented in Fig. 7.1(b).

\section{Repercussions on the Riemann zeta function}

The Riemann zeta function

$$
\zeta(s)=\sum_{u=1}^{\infty} \frac{1}{u^{s}}
$$

defined for complex numbers $s$ is one of the most important mathematical objects discovered so far (see [24, 36]). It has been first introduced and studied by Euler (see $[37,38,39,40])$ who proved the famous identity

$$
\sum_{u=1}^{\infty} \frac{1}{u^{s}}=\prod_{p \text { primes }} \frac{1}{1-p^{-s}}
$$

being one of the strongest sources of the interest to the Riemann zeta function. Many interesting results have been established for this function (see [24, 36] for a comprehensive discussion). In the context of this paper, the following results will be of our primary interest.

Traditionally, it is said that $\zeta(s)$ diverges on the open half-plane of $s$ such that the real part $\Re(s)<1$. Then, for $s=0$ the following value is attributed to $\zeta(0)$

$$
\zeta(0)=\sum_{u=1}^{\infty} \frac{1}{u^{0}}=1+1+1+\ldots=-\frac{1}{2}
$$

and the following relation

$$
\eta(s)=\left(1-2^{1-s}\right) \zeta(s)
$$


links the Riemann zeta function to the Dirichlet eta function

$$
\eta(s)=\sum_{u=1}^{\infty} \frac{(-1)^{u-1}}{u^{s}} .
$$

It has been shown for the Riemann zeta function that it has trivial zeros at the points $-2,-4, \ldots$ It is also known that any non-trivial zero lies in the complex set $\{s: 0<\Re(s)<$ $1\}$, called the critical strip. The complex set $\{s: \mathfrak{R}(s)=1 / 2\}$ is called the critical line. The Riemann Hypothesis asserts that any non-trivial zero $s$ has the real part $\Re(s)=1 / 2$, i.e., lies on the critical line.

In this section, we study the Riemann zeta function in the framework of the (1)-based approach using strongly results from sections 6.2 and 6.3 discussing how divergent series are substituted by sums with a fixed infinite number of addends.

The first observation that can be done consists of the fact that thanks to the introduction of (1) within $\infty$ many different infinite numbers can be distinguished. Therefore, records (8.1) and (8.5) do not describe two single functions and there exist many different Riemann zeta functions and many different Dirichlet eta functions and to fix a concrete one it is necessary to fix the number of addends in the corresponding sum (see section 6.2). In order to define a Riemann zeta function (a Dirichlet eta function), we should choose an infinite number $n$ expressible in a numeral system using grossone and then write

$$
\zeta(s, n)=\sum_{u=1}^{n} \frac{1}{u^{s}}, \quad \eta(s, n)=\sum_{u=1}^{n} \frac{(-1)^{u-1}}{u^{s}} .
$$

As a result, for different (infinite or finite) values of $n$ we have different functions. For example, for $n=(1 / 2$ and $n=(1)$ we get

$$
\zeta(s, 1 / 2)=\sum_{u=1}^{\mathbb{\Phi} / 2} \frac{1}{u^{s}}, \quad \zeta(s,(1))=\sum_{u=1}^{\oplus} \frac{1}{u^{s}}
$$

that are two different functions and, analogously,

$$
\eta(s, \mathbb{1})=\sum_{u=1}^{\mathbb{D}} \frac{(-1)^{u-1}}{u^{s}}, \quad \eta(s, \mathbb{1} / 2)=\sum_{u=1}^{\mathbb{\Phi} / 2} \frac{(-1)^{u-1}}{u^{s}},
$$

are also two different functions.

It is necessary to stress that $(8.1),(8.5)$ on the one hand and (8.6) on the other hand describe the same mathematical objects - sums with an infinite number of addends - but do it with different accuracies. In the case (8.1), (8.5), the numeral $\infty$ is used and the number of addends is not fixed - it is just said that it is infinite. The (1)-based lens allows us to observe that both series are not single objects with a generic infinite number of addends expressed by the numeral $\infty$. Records (8.1) and (8.5) were visible like unique objects due to the low accuracy of the numeral $\infty$. Now we are able distinguish different sums in (8.6) and for different infinite values of $n$ different results can be obtained.

Notice also that it would not be correct to say that the record (8.1) represents a 'true Riemann zeta function that includes all possible addends $\frac{1}{u^{s}}$, whereas (8.6), where the 
number of summands should be expressed by concrete infinite (1)-based numbers, is just 'a partial infinite sum'. As was already discussed in section 6.2, the sentence 'all possible addends' means that the numeral $\infty$ being the upper index of summation represents the largest possible integer (if it is not the largest one then we do not consider all possible addends). Thus, the operation $\infty+1$ should be prohibited since we are not allowed to increase $\infty$ that represents the largest possible integer and the operation $\infty-1$ should give a result different from $\infty$ since in this case we have less addends in the sum. In other words, the assumption that (8.1) includes all possible addends $\frac{1}{u^{s}}$ requires by necessity to attribute to the symbol $\infty$ properties that are different from its traditional usage, where relations $\infty+1=\infty$ and $\infty-1=\infty$, etc. hold. Therefore, the meaning 'sum of all possible addends $\frac{1}{u^{s}}$ ' cannot be used if the numeral $\infty$ is applied in its conventional sense. Thus, records (8.1), (8.5) on the one hand and (8.6), on the other hand, describe the same mathematical objects - sums with an infinite number of addends - but do it with different accuracies.

Notice that there obviously exist questions for which both mathematical languages used in (8.1) and (8.6) are sufficiently accurate. This can happen when the property we ask about holds for all (finite and infinite) values of $n$ in (8.6). For instance, it is possible to answer in the affirmative to the question: 'Is the inequality $\zeta(1)>-100$ correct?' because this result holds for all values of $n$ in (8.6) and, therefore, the accuracy of the record (8.1) allows us to answer in this case.

8.1. Some Euler's results revisited in the (1)-based framework. Let us consider some of results of Euler related to the Riemann zeta function (8.1) from the new methodological positions in order to re-write them (where it is possible) for the form (8.6) with concrete infinite values of $n$. First, the relation (8.4) can be re-written as

$$
\eta(s, n)=\zeta(s, 2 k)-2^{1-s} \zeta(s, k)
$$

for $n=2 k$ and as

$$
\eta(s, n)=\zeta(s, 2 k+1)-2^{1-s} \zeta(s, k)
$$

for $n=2 k+1$.

Let us look now at the formula (8.2) that was introduced and studied by Euler (see $[37,38,39,40])$ who proved it for integer values of $s$. This identity has been proved using the traditional language and, as a consequence, its accuracy is not sufficient to distinguish within $\infty$ different infinite numbers. If one uses the (1)-based language, then identities involving infinite sums and/or products of the type

$$
\sum_{u=1}^{\infty} a_{i}=\prod_{i=1}^{\infty} b_{i}
$$

should be substituted, where this is possible, by identities

$$
\sum_{u=1}^{n} a_{i}=\prod_{i=1}^{k} b_{i}
$$

where both $n$ and $k$ are fixed (and possibly different) infinite numbers. As a consequence, proofs of such identities should be reconsidered with the accuracy imposed by the new 
language. It can easily happen that a result, while being correct with the accuracy of one language is not sufficiently precise when a language with a higher accuracy is used (see discussion on the accuracy of the numeral system of Pirahã in the bottom of page 8). The introduction of numerals 3 and 4 allows us to abandon the results $2+1=$ 'many', $2+2=$ 'many' and to see that $2+1 \neq 2+2$. Analogously, the passage from the low accuracy induced by the symbol $\infty$ to more accurate (1)-based numerals can lead to situations where the correctness of (8.9) does not imply the correctness of (8.10).

Let us compare the usage of the traditional language working with $\infty$ and the new one using (1) with respect to the Euler product formula (8.2). To prove it, Euler expands each of the factors of the right-hand part of (8.2) as follows

$$
\frac{1}{1-\frac{1}{p^{s}}}=1+\frac{1}{p^{s}}+\frac{1}{\left(p^{2}\right)^{s}}+\frac{1}{\left(p^{3}\right)^{s}}+\ldots
$$

Then he observes that their product is therefore a sum of terms of the form

$$
\frac{1}{\left(p_{1}^{n_{1}} \cdot p_{2}^{n_{2}} \cdot \ldots \cdot p_{r}^{n_{r}}\right)^{s}},
$$

where $p_{1}, p_{2}, \ldots p_{r}$ are distinct primes and $n_{1}, n_{2}, \ldots n_{r}$ are natural numbers. Euler then uses the fundamental theorem of arithmetic (every integer can be written as a product of primes) and concludes that the sum of all items (8.12) is the left-hand part of (8.2).

In the new language this way of doing things is not acceptable because, in order to start, we should indicate the precise infinite number, $n$, of items in the sum in the left-hand part of (8.2). This operation gives us different functions $\zeta(s, n)$ from (8.6). Then (8.11) should be rewritten by indicating the exact infinite number, $k$, of items in its right hand part and the result of summing up will be different with respect to (8.2). Namely, we have

$$
1+\frac{1}{p^{s}}+\frac{1}{\left(p^{2}\right)^{s}}+\frac{1}{\left(p^{3}\right)^{s}}+\ldots+\frac{1}{\left(p^{k-1}\right)^{s}}=\frac{1-\frac{1}{\left(p^{k}\right)^{s}}}{1-\frac{1}{p^{s}}} .
$$

The accuracy of the language used by Euler did not allow him either to observe different infinite values of $n$ and $k$ or to take into account the infinitesimal value $\frac{1}{\left(p^{k}\right)^{s}}$. His results are correct but their accuracy is low.

Another metaphor that can help is the following. Suppose that we have measured two distances $A$ and $B$ with the accuracy equal to 1 meter and we have found that both of them are equal to 25 meters. Suppose now that we want to measure them with the accuracy equal to 1 centimeter. Then, very probably, we shall obtain something like $A=2487$ centimeters and $B=2538$ centimeters, i.e., $A \neq B$. Both answers, $A=B$ and $A \neq B$, are correct but with different accuracies and both of them can be used successfully in different situations. For instance, if one just wants to go for a walk, then the accuracy of the answer $A=B$ expressed in meters is sufficient. However, if one needs to connect some devices with a cable, then a higher accuracy is required and the answer expressed in centimeters should be used.

We are with the Euler product formula in the same situation. It is correct with the accuracy of the language using $\infty$ because this language does not allow one to distinguish 
different infinite numbers within $\infty$. At the same time, when a language allows us to distinguish different infinite and infinitesimal numbers, it follows from (8.13) that for any infinite (or finite) $k$ we have

$$
1+\frac{1}{p^{s}}+\frac{1}{\left(p^{2}\right)^{s}}+\frac{1}{\left(p^{3}\right)^{s}}+\ldots+\frac{1}{\left(p^{k-1}\right)^{s}} \neq \frac{1}{1-p^{-s}}
$$

and, therefore, the following theorem holds (a similar result obtained using a different reasoning can be found in [114]).

Theorem 8.1. For prime $p_{i}$ and both finite and infinite values of $n$ and $k$ it follows that

$$
\sum_{u=1}^{n} \frac{1}{u^{s}} \neq \prod_{i=1}^{k} \frac{1}{1-p_{i}^{-s}}
$$

Let us comment now upon another famous result of Euler related to the Riemann zeta function - his solution to the Basel problem where he has shown that

$$
1+\frac{1}{2^{2}}+\frac{1}{3^{2}}+\frac{1}{4^{2}}+\ldots=\frac{\pi^{2}}{6}
$$

Let us first briefly present Euler's proof and then comment upon it. Note that from the point of view of modern Mathematics this proof is amenable to criticism from different points of view. Nowadays there exist many other proofs considered by mathematicians to be more accurate ${ }^{14}$. However, since also in modern proofs the symbol $\infty$ and the concept of series are used, the analysis made below can be applied to these proofs, as well.

Euler begins with the standard Taylor expansion of $\sin (x)$,

$$
\sin (x)=x-\frac{x^{3}}{3 !}+\frac{x^{5}}{5 !}-\frac{x^{7}}{7 !}+\ldots
$$

which converges for all $x$. Euler interprets the right-hand side of (8.17) as an infinite polynomial' $P(x)$ that, therefore, can be written as product of factors based on its roots. Since the roots of $\sin (x)$ are $\ldots-3 \pi,-2 \pi,-\pi, 0, \pi, 2 \pi, 3 \pi, \ldots$ then it follows

$$
P(x)=C x\left(x^{2}-\pi^{2}\right)\left(x^{2}-4 \pi^{2}\right)\left(x^{2}-9 \pi^{2}\right) \ldots
$$

Euler continues by recalling that $\lim _{x \rightarrow 0} \frac{\sin (x)}{x}=1$ and

$$
\lim _{x \rightarrow 0} \frac{x\left(x^{2}-\pi^{2}\right)\left(x^{2}-4 \pi^{2}\right)\left(x^{2}-9 \pi^{2}\right) \ldots}{x}=\left(-\pi^{2}\right)\left(-4 \pi^{2}\right)\left(-9 \pi^{2}\right) \ldots
$$

Thus, $C$ should be the reciprocal of the infinite product on the right and we obtain

$$
\sin (x)=x\left(1-\frac{x^{2}}{\pi^{2}}\right)\left(1-\frac{x^{2}}{2^{2} \pi^{2}}\right)\left(1-\frac{x^{2}}{3^{2} \pi^{2}}\right) \ldots
$$

\footnotetext{
${ }^{14}$ This fact is another manifestation of the continuous mutation of mathematical languages. The views on accuracy of proofs have changed since Euler's times and the modern language is considered to be more accurate.
} 
Then, as follows from (8.17) and (8.18), Euler has written $P(x)$ in two different ways and he equates these two records

$$
\sin (x)=x-\frac{x^{3}}{3 !}+\frac{x^{5}}{5 !}-\frac{x^{7}}{7 !}+\ldots=x\left(1-\frac{x^{2}}{\pi^{2}}\right)\left(1-\frac{x^{2}}{2^{2} \pi^{2}}\right)\left(1-\frac{x^{2}}{3^{2} \pi^{2}}\right) \ldots,
$$

Now Euler equates the coefficients of $x^{3}$ on both sides of (8.18) and gets first

$$
-\frac{1}{3 !}=-\frac{1}{\pi^{2}}-\frac{1}{2^{2} \pi^{2}}-\frac{1}{3^{2} \pi^{2}}-\frac{1}{4^{2} \pi^{2}} \ldots
$$

and then the final beautiful result (8.16).

Let us now consider these results using the (1)-based approach. We take the function $\sin (x)$ introduced using the standard trigonometric reasoning. Note that such a definition just describes its properties and does not tell us how to calculate $\sin (x)$ precisely at all $x$. When one uses the trigonometric definition, values of $\sin (x)$ only at certain $x$ are known precisely, e.g., $\sin \left(\frac{\pi}{2}\right)=1, \sin (2 \pi)=0$, etc. Notice that in these records we do not use any approximation of the number $\pi$. Similarly to $\sin (x)$, it is described by its properties and a special numeral, $\pi$, is introduced to indicate it. Then, those values of $\sin (x)$ that are not linked to geometric ideas are defined through various approximations of both $\sin (x)$ and $\pi$.

The (1)-based numerals taken together with the trigonometric definition of $\sin (x)$ allow us to evaluate $\sin (x)$ precisely not only at certain finite points but also at certain infinite points. For instance, it follows that $\left.\sin (21) \pi)=0, \sin (1) \pi+\frac{\pi}{2}\right)=1$, etc.

Then, the explicit usage of infinite and infinitesimal numbers requires that we move from (8.17) to the approximation with the polynomial $P_{1}(x, 2 k+1)$ of the order $2 k+1$ where

$$
\sin (x) \approx P_{1}(x, 2 k+1)=x-\frac{x^{3}}{3 !}+\frac{x^{5}}{5 !}-\frac{x^{7}}{7 !}+\ldots+(-1)^{k} \frac{x^{2 k+1}}{(2 k+1) !},
$$

and for different finite or infinite $k$ we get different approximations. Analogously, the idea used in (8.18) gives us the second kind of approximation where the polynomial $P_{2}(x, 2 n+$ 1) of the order $2 n+1$ is used

$$
\begin{aligned}
\sin (x) \approx P_{2}(x, 1) & =x, \\
\sin (x) \approx P_{2}(x, 2 n+1) & =x\left(1-\frac{x^{2}}{\pi^{2}}\right)\left(1-\frac{x^{2}}{2^{2} \pi^{2}}\right) \ldots\left(1-\frac{x^{2}}{n^{2} \pi^{2}}\right),
\end{aligned}
$$

where for different finite or infinite values of $n$ we get different approximations.

Obviously, when $k \neq n$, it follows $P_{1}(x, 2 k+1) \neq P_{2}(x, 2 n+1)$. In the case $k=n$, two polynomials will be equal if all their coefficients are equal. Following Euler we first equate the coefficients of $x^{3}$ and obtain

$$
-\frac{1}{3}=-\frac{1}{\pi^{2}}-\frac{1}{2^{2} \pi^{2}}-\frac{1}{3^{2} \pi^{2}}-\frac{1}{4^{2} \pi^{2}} \ldots-\frac{1}{k^{2} \pi^{2}} .
$$

From where we get

$$
\frac{\pi^{2}}{6}=1+\frac{1}{2^{2}}+\frac{1}{3^{2}}+\frac{1}{4^{2}}+\ldots+\frac{1}{k^{2}}
$$




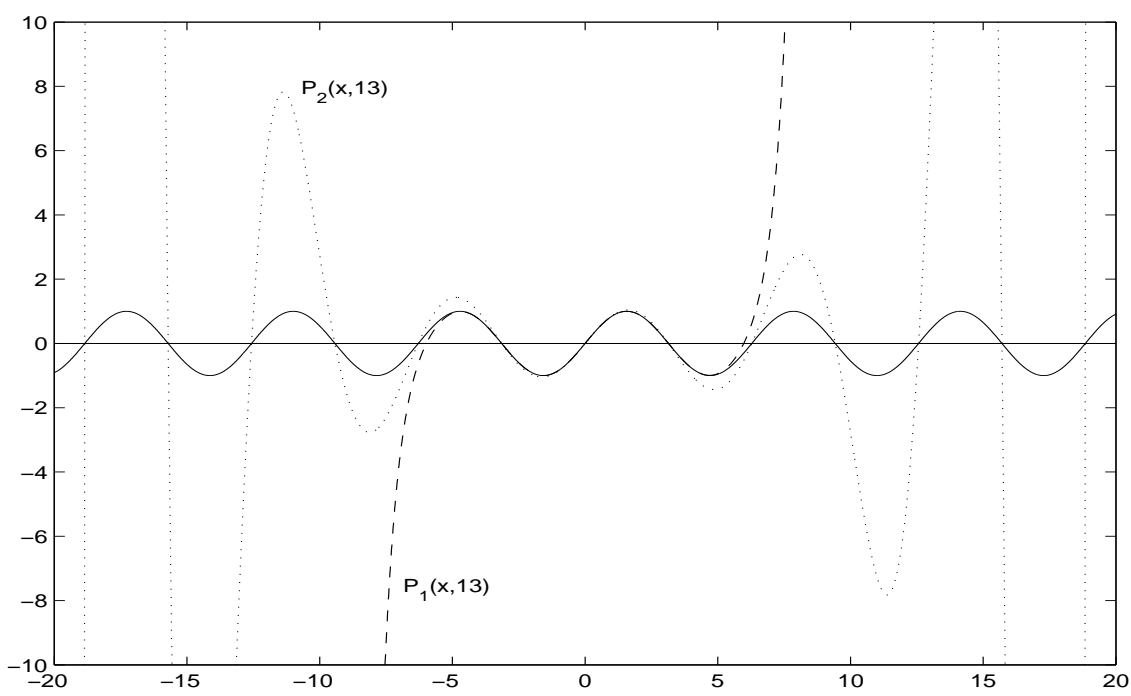

Figure 8.1. Polynomials $P_{1}(x, 13)$ and $P_{2}(x, 13)$ together with $\sin (x)$.

However, such a kind of equating should be done for all the coefficients of $P_{1}(x, 2 k+1)$ and $P_{2}(x, 2 n+1)$. Suppose that $k$ in the sum (8.20) is even, by equating the coefficients of the highest power $2 k+1$ of $x$ in two polynomials we get that it should be

$$
\frac{1}{(2 k+1) !}=\frac{1}{(k !)^{2} \pi^{2 k}},
$$

whence we deduce

$$
\frac{\pi^{2 k}}{(2 k+1) !}=\frac{1}{(k !)^{2}} .
$$

Equating coefficients for other powers of $x$ will give us several different expressions to be satisfied and it is easy to see that they cannot all simultaneously hold.

Thus, polynomials $P_{1}(x, 2 k+1)$ and $P_{2}(x, 2 n+1)$ give us different approximations of $\sin (x)$. As an example we show in Fig. 8.1 polynomials $P_{1}(x, 13)$ and $P_{2}(x, 13)$ together with $\sin (x)$. The higher is the order of the polynomials the better are the approximations but the differences are always present for both finite and infinite values of $k$ and $n$.

As it was with the product formula, Euler results are correct with the accuracy of the traditional mathematical language working only with the symbol $\infty$. Such language does not allow one to distinguish different infinite values of $k$ and $n$ and, as a consequence, different infinite polynomials $P_{1}(x, 2 k+1)$ and $P_{2}(x, 2 n+1)$.

Since all the traditional computations are executed with finite values of $k$ and $n$ and only the initial finite part of (8.23) is used, it is always possible to choose sufficiently high finite values of $k$ and $n$ yielding valid approximations of $\pi$ with a finite accuracy. We are not able by using the traditional mathematical tools to observe the behavior of the polynomials and $\sin (x)$ at various points $x$ at infinity. Thus, the fact of the difference of 
these objects in infinity cannot be detected by finite numeral systems extended only by the symbol $\infty$. However, it is perfectly visible when one uses the new numeral system where different infinite and infinitesimal numbers can be expressed and distinguished. Thus, we can conclude this subsection by the following theorem that has just been proved.

Theorem 8.2. For both finite and infinite values of $n$ and $k$ it follows that

$$
\sin (x) \neq P_{1}(x, 2 k+1) \neq P_{2}(x, 2 n+1) .
$$

8.2. Calculating $\zeta(s, n)$ for different infinite values of $n$ at $s=0,-1,-2$, etc. In this subsection, we establish some results for functions $\zeta(s, n)$ and $\eta(s, n)$ from (8.6) using the approach presented in sections 6.2 and 6.3 where it was shown how to move from series to sums with a fixed infinite number of addends. The analysis made there allows us to calculate $\zeta(s, n)$ and $\eta(s, n)$ for infinite values of $n$ at $s=0$ and $s=-1$ :

$$
\begin{gathered}
\zeta(0, n)=\underbrace{1+1+1+\ldots+1}_{n \text { addends }}=n, \\
\zeta(-1, n)=1+2+3+\ldots+n=\frac{(n+1) n}{2} .
\end{gathered}
$$

For instance, at $n=(1) / 2$ and $n=(1)$ we obtain

$$
\begin{gathered}
\zeta(0,1 / 2)=1 / 2, \quad \zeta(0,1)=1, \\
\zeta(-1,1 / 2)=\frac{(1 / 2+1)(1)}{4}, \quad \zeta(-1,1)=\frac{(1)+1)(1)}{2} .
\end{gathered}
$$

Analogously, calculation of the function, $\eta(s, n)$, at the points $s=0$ and $s=-1$ for infinite values of $n$ also has been discussed in section 6.3. We obtain

$$
\eta(0, n)=\underbrace{1-1+1-1+1-1+1-\ldots}_{n \text { addends }}= \begin{cases}0, & \text { if } n=2 k, \\ 1, & \text { if } n=2 k+1,\end{cases}
$$

that gives, for example, $\eta(0,1)=0$ and $\left.\eta(0,1)^{2}-1\right)=1$. In section 6.3 we have seen also how it is possible to calculate $\eta(-1, n)$ for infinite values of $n$. For instance, it follows that $\eta(-1,1)=-\frac{1}{2}$. Notice that these results fit well with relation (8.7). For example, for $n=(1)$ we have

$$
\begin{gathered}
\zeta(0,1)-2 \zeta(0,1) / 2)=0=\eta(0,1), \\
\zeta(-1,1)-4 \zeta(-1,1 / 2)=-\frac{1}{2}=\eta(-1,1) .
\end{gathered}
$$

Let us present now a general method for calculating values of $\zeta(s, n)$ and $\eta(s, n)$ for infinite or finite $n$ at $s$ being negative finite integers. For this purpose we use the idea of Euler to multiply the identity

$$
f(x)=\sum_{i=0}^{n} x^{i}=1+x+x^{2}+\ldots+x^{n}
$$


by $x$. By using the differential calculus developed in [91] we can apply this idea without distinction for both finite and infinite values of $n$ and $x \neq 1$ that can be finite, infinite or infinitesimal. By differentiation of (8.25) we obtain

$$
f^{\prime}(x)=\sum_{i=0}^{n-1} i x^{i-1}=1+2 x+3 x^{2}+\ldots+n x^{n-1},
$$

Since we have for finite, infinite or infinitesimal $x \neq 1$ that

$$
f(x)=\frac{1-x^{n+1}}{1-x}
$$

then by differentiation of (8.27) we have that

$$
f^{\prime}(x)=\frac{1+n x^{n+1}-(n+1) x^{n}}{(1-x)^{2}} .
$$

Thus, we can conclude that for finite and infinite $n$ and for finite, infinite or infinitesimal $x \neq 1$ it follows that

$$
\frac{1+n x^{n+1}-(n+1) x^{n}}{(1-x)^{2}}=\sum_{i=0}^{n-1} i x^{i-1}=1+2 x+3 x^{2}+\ldots+n x^{n-1} .
$$

Taking $x=-1$ we obtain that the value of $\eta(-1, n)$ is

$$
\eta(-1, n)=1-2+3-4+\ldots+n(-1)^{n-1}=\frac{1+n(-1)^{n+1}-(n+1)(-1)^{n}}{4} .
$$

For example, for $n=(1$ we obtain the result that has been already calculated in section 6.3 in a different way

$$
\begin{gathered}
\eta(-1,1)=1-2+3-4+\ldots+(1)(-1)^{\circledR}-1= \\
\frac{1+(1)(-1)^{\circledR+1}-(1+1)(-1)^{\circledR}}{4}=\frac{1-(1-(1)+1)}{4}=-\frac{1}{2} .
\end{gathered}
$$

If we multiply successively (8.29) by $x$ and use again differentiation of both parts of the obtained equalities then it becomes possible to obtain the values of $\eta(s, n)$ for other finite integer negative points $s$. Thus, in order to obtain, for instance, formulae for $s=$ $-2,-3,-4$, we proceed as follows

$$
\begin{gathered}
1+2^{2} x+3^{2} x^{2}+\ldots+n^{2} x^{n-1}=\frac{-n^{2} x^{n+2}+\left(2 n^{2}+2 n-1\right) x^{n+1}-(n+1)^{2} x^{n}+x+1}{(1-x)^{3}}, \\
1+2^{3} x+3^{3} x^{2}+4^{3} x^{3} \ldots+n^{3} x^{n-1}=\left(n^{3} x^{n+3}-\left(3 n^{3}+3 n^{2}-3 n+1\right) x^{n+2}+\right. \\
\left.\left(3 n^{3}+6 n^{2}-4\right) x^{n+1}-(n+1)^{3} x^{n}+x^{2}+4 x+1\right)(1-x)^{-4} \\
1+2^{4} x+3^{4} x^{2}+4^{4} x^{3} \ldots+n^{4} x^{n-1}=\left(n^{4} x^{n+4}-\left(4 n^{4}+4 n^{3}-6 n^{2}+4 n-1\right) x^{n+3}+\right.
\end{gathered}
$$




$$
\begin{gathered}
\left(6 n^{4}+12 n^{3}-6 n^{2}-12 n+11\right) x^{n+2}-\left(4 n^{4}+12 n^{3}+6 n^{2}-12 n-11\right) x^{n+1}+ \\
\left.(n+1)^{4} x^{n}-x^{3}-11 x^{2}-11 x-1\right)(x-1)^{-5} .
\end{gathered}
$$

Taking $x=-1$ in (8.30), (8.31), and (8.32) we obtain that

$$
\begin{gathered}
\eta(-2, n)=2^{-3}\left(-n^{2}(-1)^{n+2}+\left(2 n^{2}+2 n-1\right)(-1)^{n+1}-(n+1)^{2}(-1)^{n}\right), \\
\eta(-3, n)=2^{-4}\left(n^{3}(-1)^{n+3}-\left(3 n^{3}+3 n^{2}-3 n+1\right)(-1)^{n+2}+\right. \\
\left.\left(3 n^{3}+6 n^{2}-4\right)(-1)^{n+1}-(n+1)^{3}(-1)^{n}-2\right), \\
\eta(-4, n)=-2^{-5}\left(n^{4}(-1)^{n+4}-\left(4 n^{4}+4 n^{3}-6 n^{2}+4 n-1\right)(-1)^{n+3}+\right. \\
\left(6 n^{4}+12 n^{3}-6 n^{2}-12 n+11\right)(-1)^{n+2}-\left(4 n^{4}+12 n^{3}+6 n^{2}\right. \\
\left.-12 n-11)(-1)^{n+1}+(n+1)^{4}(-1)^{n}\right) .
\end{gathered}
$$

Then, by taking different infinite values of $n$ we are able to calculate the respective values of the functions. For example, for $n=(1)$ it follows that

$$
\begin{aligned}
& \left.\left.\left.\left.\eta(-2,1)=2^{-3}(-1)^{2}-21^{2}-21\right)+1-(1)+1\right)^{2}\right)=-0.5(1)(1)+1\right), \\
& \left.\left.\eta(-3,1)=2^{-4}(-1)^{3}-(31)^{3}+31\right)^{2}-31+1\right)- \\
& \left.\left.\left(31^{3}+6(1)^{2}-4\right)-(1)+1\right)^{3}-2\right)=-0.5(1)^{2}(1+3), \\
& \left.\eta(-4,1)=-2^{-5}(1)^{4}+4(1)^{4}+4(1)^{3}-6(1)^{2}+411-1+61\right)^{4}+12(1)^{3}- \\
& \left.\left.6(1)^{2}-12(1)+11+4(1)^{4}+12(1)^{3}+6(1)^{2}-12(1)-11+(1)+1\right)^{4}\right)= \\
& -0.51(1)+1)(1)^{2}+(1-1) \text {. }
\end{aligned}
$$

In order to use the same technique to calculate $\zeta(s, n)$ for infinite or finite $n$ at negative finite integers $s<-1$ it would be necessary to evaluate (8.30), (8.31), and (8.32) at the point $x=1$. In traditional Mathematics this is impossible whereas the new approach (see $[90,91])$ allows us to execute the required evaluations by using the following method.

If we put $x=1$ at the left-hand parts of (8.30), (8.31), and (8.32) then we see that we have there infinite sums of positive integers. Thus, these sums should be equal to some infinite positive integers. Since in the right-hand parts of these equalities it is not possible to use $x=1$, we introduce an infinitesimal perturbation, ${ }^{-\alpha}, \alpha>0$, and calculate the right-hand parts at the point $x=1+1^{-\alpha}$ that is infinitely close to $x=1$. Then, in the obtained result, we separate the contribution of the perturbation that can be kept infinitesimal by the choice of $\alpha$, from the contribution of the point $x=1$ that should be equal, as we have established, to an infinite integer.

In order to illustrate the method, let us execute calculations for $s=-2$, results for other values of $s$ are obtained by a complete analogy. We indicate the right-hand part of (8.30) as $f(x, n)$ and, by using the usual notation, $\left(\begin{array}{l}n \\ k\end{array}\right)$, for binomial coefficients, proceed as follows

$$
f\left(1+1^{-\alpha}, n\right)=-1^{3 \alpha}\left(-n^{2}\left[1+(n+2) 1^{-\alpha}+\frac{1}{2}(n+1)(n+2) 1^{-2 \alpha}+\right.\right.
$$




$$
\begin{gathered}
\left.\left.\frac{1}{6} n(n+1)(n+2) 1^{-3 \alpha}+\left(\begin{array}{c}
n+2 \\
4
\end{array}\right) 1^{-4 \alpha}+\ldots+1\right)^{-(n+2) \alpha}\right]+ \\
\left(2 n^{2}+2 n-1\right)\left[1+(n+1) 1^{-\alpha}+\frac{1}{2} n(n+1)(1)^{-2 \alpha}+\frac{1}{6}(n-1) n(n+1) 1^{-3 \alpha}+\right. \\
\left.\left.\left(\begin{array}{c}
n+1 \\
4
\end{array}\right)(1)^{-4 \alpha}+\ldots+1\right)^{-(n+1) \alpha}\right]-(n+1)^{2}\left[1+n\left(1^{-\alpha}+\frac{1}{2}(n-1) n(1)^{-2 \alpha}+\right.\right. \\
\left.\frac{1}{6}(n-2)(n-1) n 1^{-3 \alpha}+\left(\begin{array}{c}
n \\
4
\end{array}\right)\left(1^{-4 \alpha}+\ldots+(1)^{-n \alpha}\right]+(1)^{-\alpha}+2\right) .
\end{gathered}
$$

By collecting the terms of grossone we then obtain

$$
\begin{gathered}
f\left(1+1^{-\alpha}, n\right)=\frac{1}{6} n(n+1)(2 n+1)+ \\
\left(n^{2}\left(\begin{array}{c}
n+2 \\
4
\end{array}\right)-\left(2 n^{2}+2 n-1\right)\left(\begin{array}{c}
n+1 \\
4
\end{array}\right)+(n+1)^{2}\left(\begin{array}{c}
n \\
4
\end{array}\right)\right)(1)^{-\alpha}+\ldots+n^{2}(1)^{-(n+2) \alpha} .
\end{gathered}
$$

As it can be seen from (8.36), for any finite or infinite value of $n$ there always can be chosen a number $\alpha>0$ such that the contribution of the added infinitesimal $1^{-\alpha}$ in $f(1+$ $\left.1^{-\alpha}, n\right)$ is a sum of infinitesimals (see the second line of (8.36)). Due to the representation (4.16), (4.17), this contribution can be easily separated from the integer finite or infinite part represented by the first line of (8.36).

Thus, we have obtained that for finite and infinite values of $n$ it follows

$$
\zeta(-2, n)=\frac{1}{6} n(n+1)(2 n+1) .
$$

Analogously, by applying the same procedure again we can obtain the formulae for $\zeta(s, n)$ for other finite integer negative points $s$. For instance,

$$
\begin{gathered}
\zeta(-3, n)=\frac{1}{4} n^{2}(n+1)^{2}, \\
\zeta(-4, n)=\frac{1}{30} n(n+1)(2 n+1)\left(3 n^{2}+3 n-1\right) .
\end{gathered}
$$

Note that the previous results fit perfectly both well-known formulae for finite values of $n$ (see [8]) and relation (8.7). For example, using (8.7) and formulae (8.37), (8.38), and (8.39) with $n=(1)$ and $n=(1) / 2$ we obtain (cf. (8.33), (8.34), and (8.35)) that

$$
\begin{gathered}
\left.\left.\left.\zeta(-2,1)-8 \zeta(-2,1 / 2)=\frac{1}{6}(1)+1\right)(21+1)-\frac{1}{3}(1)+2\right)(1)+1\right)= \\
-0.51(1)+1)=\eta(-2,1), \\
\left.\left.\zeta(-3,1)-16 \zeta(-3,1) / 2)=\frac{1}{4} 1^{2}(1)+1\right)^{2}-(1)^{2}(0.51)+1\right)^{2}= \\
\left.-0.5(1)^{2}(1)+3\right)=\eta(-3,1), \\
\left.\left.\zeta(-4,1)-32 \zeta(-4,1 / 2)=\frac{1}{30}(1)+1\right)(21+1)(31)^{2}+31-1\right)-
\end{gathered}
$$


$\left.\left.\left.\left.\left.\frac{1}{15}(1)(1)+2\right)(1)+1\right)(31)^{2}+6(1)-4\right)=-0.5(1)(1)+1\right)(1)^{2}+(1)-1\right)=\eta(-4,(1)$.

The analysis done in this section shows that the traditional mathematical language using the symbol $\infty$ very often does not possess a sufficiently high accuracy when one deals with problems having their interesting properties at infinity. For instance, it does not allow us to distinguish within the record $f(x)=\sum_{i=1}^{\infty} a_{i}(x)$ different functions $f_{n}(x)=$ $\sum_{i=1}^{n} a_{i}(x)$ emerging for different infinite $n$. However, functions $f_{n}(x)$ become visible if one uses more powerful numeral systems allowing one to represent different infinite (and infinitesimal) numbers. Then, it follows that if $a_{n+1}(x) \neq 0$ functions $f_{n}(x)$ and $f_{n+1}(x)$ are different and $f_{n}(x) \neq f_{n+1}(x)$. When $a_{n+1}(x)$ is an infinitesimal number then the difference $f_{n}(x)-f_{n+1}(x)$ is also infinitesimal, i.e., invisible if one uses the traditional mathematical language but perfectly visible through the (1)-based numeral system.

In particular, we have seen that the Riemann Hypothesis asks a question about the behavior of a function defined traditionally using the symbol $\infty$. As we can see using the (1)-based methodology and the respective numeral system distinguishing different infinite numbers, the Riemann zeta function is not a function but many different functions that are indistinguishable if one uses the traditional mathematics. The analysis of the Riemann zeta function made in the traditional style does not consider various infinite and infinitesimal numbers that are crucial not only in the context of the Hypothesis but even with respect to the definition of the function itself. It has been shown that several classical results do not represent properties of the the Riemann zeta function but reflect defects of the numeral system using the symbol $\infty$. Of course, there remains a possibility that the question asked in the Hypothesis has the same answer for all infinite $n$. Unfortunately, this is impossible due to the analysis made above and the analysis of the partial zeta functions with a finite number of addends made in [4, 11, 108].

We conclude this subsection by the following remark. It is well known that the Riemann zeta function appears in many different mathematical contexts and there exist many different equivalent formulations of the Riemann hypothesis. In view of the above analysis, this can be explained again by the accuracy of the traditional mathematical language. Such phenomena indicate situations where its accuracy is not sufficient. By returning to the metaphor of the microscope, we can say that out traditional lens is too weak to distinguish different mathematical objects observed in these situations. Thus, these mathematical contexts can be viewed as very promising for obtaining new results by applying the new numeral system that does not use the symbol $\infty$ and allows one to distinguish and to treat numerically various infinite and infinitesimal numbers.

\section{Grossone for studying probability and fractals}

9.1. Probability and absence of nonempty sets of measure zero. A formalization of the concept 'point' introduced above allows us to execute more accurately computations having relations to this concept. In this subsection, we show that the (1)-based approach allows us to distinguish the impossible event having probability equal to zero (i.e., $P(\varnothing)=$ 0 ) and events having infinitesimal probabilities. Moreover, the introduction of the notion 


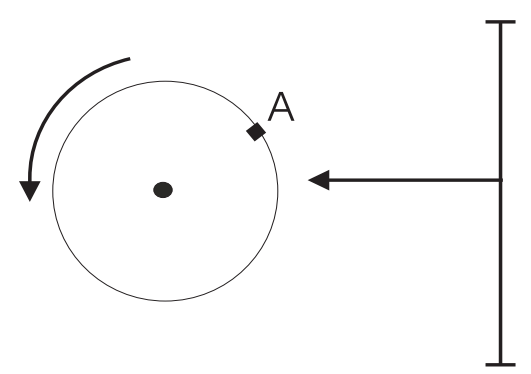

Figure 9.1. What is the probability that the rotating disk stops in such a way that the point $A$ will be exactly in front of the arrow?

of accuracy of numeral systems allows us to avoid the occurrence of nonempty sets of measure zero in computations.

Let us consider the problem presented in Fig. 9.1 from the traditional point of view of probability theory. A disk of radius $r$, with point $A$ marked on its edge, is rotating and we would like to know the probability $P(E)$ of the following event $E$ : the disk stops in such a way that the point $A$ will be exactly in front of the arrow fixed on the wall. Since the point $A$ is an entity that has no extent traditionally $P(E)$ is calculated by considering the following limit

$$
P(E)=\lim _{h \rightarrow 0} \frac{h}{2 \pi r}=0,
$$

where $h$ is an arc of the circumference containing $A$ and $2 \pi r$ is its length. However, the point $A$ can stop in front of the arrow, i.e., this event is not impossible and its probability should be strictly greater than zero, i.e., $P(E)>0$.

In order to consider this example in the (1)-based framework, let us redescribe the experiment more rigorously by choosing a numeral system that will be used to express points on the circumference. This choice will fix the number (infinite or finite) of points, $K$, that we are able to distinguish on the circumference. This definition of the notion point allows us to define elementary events in our experiment as follows: the disk has stopped and the arrow indicates a point. As a consequence, we obtain that the number, $N(\Omega)$, of all possible elementary events, $e_{i}$, in our experiment is equal to $K$ where $\Omega=\cup_{i=1}^{N(\Omega)} e_{i}$ is the sample space of our experiment. If our disk is well balanced, all elementary events are equiprobable and, therefore, have the same probability equal to $\frac{1}{N(\Omega)}$. Thus, we can calculate $P(E)$ directly by subdividing the number, $N(E)$, of favorable elementary events by the number, $K=N(\Omega)$, of all possible events.

For example, if we use numerals of the type $\frac{i}{(1}, i \in \mathbb{N}$, then $K=1$. The number $N(E)$ depends on our decision about how many numerals we want to use to represent the point $A$. If we decide that the point $A$ on the circumference is represented by $m$ numerals, we obtain

$$
P(E)=\frac{N(E)}{N(\Omega)}=\frac{m}{K}=\frac{m}{1}>0
$$


where the number $\frac{m}{1}$ is infinitesimal if $m$ is finite. Note that this representation is interesting also from the point of view of distinguishing the notions 'point' and 'arc'. When $m$ is finite then we deal with a point, when $m$ is infinite we deal with an arc.

In case we need a higher accuracy, we could choose, for instance, numerals of the type $i\left(1^{-2}, 1 \leq i \leq 1^{2}\right.$, for expressing points at the disk. Then it follows $K=1^{2}$ and, as a result, we obtain $P(E)=m 1^{-2}>0$.

This example with the rotating disk, of course, is a particular instance of the general situation related to continuous random variables and probabilities of events corresponding to sets with measure zero. While for a discrete random variable one could say that an event with probability zero is impossible, this can not be said traditionally in the continuous case where to events corresponding to sets having measure zero the probability zero is assigned. As we have shown by the example above, the (1)-based methodology allows us to model the continuous case using infinitesimals and to avoid appearance of sets of measure zero. Thus, in both discrete and continuous cases the only event having probability zero is the impossible event.

Notice also that the obtained probabilities are not absolute, they depend on the accuracy chosen to express points on the circumference. There is again a straight analogy with Physics where it is not possible to obtain results that have a precision higher than the accuracy of the instrument used to measure data. With respect to probability, it is not possible to get a precision of computations that is higher than the precision of numerals chosen to represent elementary events. Thus, the introduction of the concept of accuracy and availability of (1)-based infinitesimals allow us to avoid appearance of non-empty sets having measure zero and to introduce the concept of relativity of probability that depends on the chosen numeral system.

9.2. Koch snowflake and its quantitative analysis using (1)-based numerals. Fractal objects have been very well studied during the last few decades (see, e.g., [41, 78] and references given therein) and have been applied in various fields (see numerous applications given in $[35,41,50,78,83,85])$. However, the mathematical analysis of fractals very often continues to have mainly a qualitative character and tools for a quantitative analysis of fractals at infinity are not very rich yet. Since fractals are objects defined as a limit of an infinite process, the computation of their dimension is one of a very few quantitative characteristics that can be calculated at infinity.

Traditionally, after $n$ iterations of a fractal process it is possible to give numerical answers to questions regarding fractals (calculation of, e.g., their length, area, volume or the number of smaller copies of initiators present at the $n$-th iteration) only for finite values of $n$. The same questions very often remain without any answer when we consider an infinite number of steps because when we speak about limit fractal objects the required values often either tend to zero and disappear, in practice, or tend to infinity, i.e., become intractable numerically. Moreover, we cannot distinguish at infinity fractals starting from similar initiators even though they are different for any fixed finite value of the iteration number $n$.

In this subsection, we propose to apply the (1) methodology to the study of fractals. In particular, the Koch snowflake being one of the first mathematically described fractals 


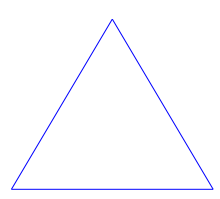

a)

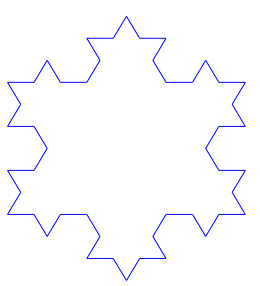

c)

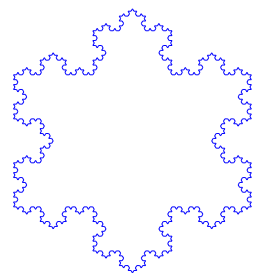

e)

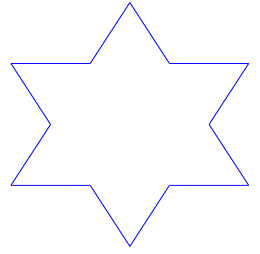

b)

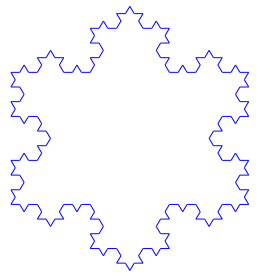

d)

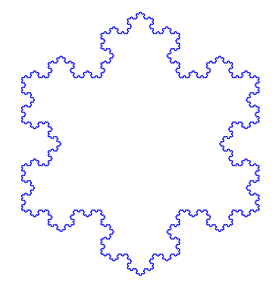

f)

Figure 9.2. Generation of the Koch snowflake.

is considered (for more texts on applying the (1) methodology to traditional and blinking fractals see $[15,87,89,97,102]$; see also papers $[68,69,70]$ related to tilings of the hyperbolic plane and grossone). The snowflake has been introduced by Helge von Koch in 1904 (see [61]). This fractal is interesting because it is known that in the limit it has an infinite perimeter but its area is finite. The procedure of its construction is shown in Fig. 9.2. The initiator (iteration number $n=0$ ) is the triangle shown in Fig. 9.2, a), more precisely, its three sides. Then each side (segment) is substituted by four smaller segments as it is shown in Fig. 9.2, b) during the first iteration, in other words, a smaller copy of the triangle is added to each side. At the iteration $n=2$ (see Fig. 9.2, c)) each segment is substituted again by four smaller segments, an so on. Thus, the Koch snowflake is the resulting limit object obtained at $n \rightarrow \infty$. It is known (see, e.g., [78]) that its fractal dimension is equal to $\frac{\log 4}{\log 3} \approx 1.26186$.

Clearly, for finite values of $n$ we can calculate the perimeter $P_{n}$ and the respective area $A_{n}$ of the snowflake. If iteration numbers $n$ and $k$ are such that $n \neq k$ then it follows $P_{n} \neq P_{k}$ and $A_{n} \neq A_{k}$. Moreover, the snowflake started from the initiator a) after $n$ iterations will 
be different from the snowflake started from the configuration b) after $n$ iterations. The simple illustration for $n=3$ can be viewed in Fig. 9.2. In fact, starting from the initiator a) after three iterations we have the snowflake d) and starting from the initiator b) after three iterations we have the snowflake e).

Unfortunately, the traditional analysis of fractals does not allow us to have quantitative answers to the questions stated above when $n \rightarrow \infty$. In fact, we know only (see, e.g., [78]) that

$$
\lim _{n \rightarrow \infty} P_{n}=\lim _{n \rightarrow \infty} \frac{4^{n}}{3^{n-1}} l=\infty, \quad \lim _{n \rightarrow \infty} A_{n}=\frac{8}{5} a_{0}, \quad a_{0}=\frac{\sqrt{3}}{4} l^{2},
$$

where $a_{0}$ is the area of the original triangle from Fig. 9.2, a) expressed in the terms of its side length $l$.

By using the (1)-based methodology a more precise quantitative analysis of the Koch snowflake can be done at infinity. In particular, it becomes possible:

- to show that at infinity the Koch snowflake is not a unique object, namely, different snowflakes can be distinguished at infinity similarly to different snowflakes that can be distinguished for different finite values of $n$;

- to calculate the exact (up to infinitesimals) perimeter $P_{n}$ of the snowflake (together with the infinite number of sides and the infinitesimal length of each side) after $n$ iterations for different infinite values of $n$;

- to show that for infinite $n$ and $k$ such that $k>n$ it follows that both $P_{n}$ and $P_{k}$ are infinite but $P_{k}>P_{n}$ and their difference $P_{k}-P_{n}$ can be computed exactly and it turns out to be infinite;

- to calculate the exact (up to infinitesimals) finite areas $A_{n}$ and $A_{k}$ of the snowflakes for infinite $n$ and $k$ such that $k>n$ and to show that it follows $A_{k}>A_{n}$, the difference $A_{k}-A_{n}$ can be also calculated exactly and it turns out to be infinitesimal;

- to show that the snowflakes constructed starting from different initiators (e.g., from initiators shown in Fig. 9.2) are different after $k$ iterations where $k$ is an infinite number, i.e., they have different infinite perimeters and different areas where the difference can be measured using infinities and infinitesimals.

Let us begin our quantitative analysis. It can be seen from Fig. 9.2 that at each iteration each side of the snowflake is substituted by 4 new sides having the length of one third of the segment that has been substituted. Thus, if we indicate as $N_{n}, n \geq 1$, the number of segments of the snowflake and as $L_{n}$ their length at the $n$-th iteration then

$$
N_{n}=4 N_{n-1}=3 \cdot 4^{n}, \quad L_{n}=\frac{1}{3} L_{n-1}=\frac{l}{3^{n}}, \quad n>1,
$$

where $l$ is the length of each side of the original triangle from Fig. 9.2. As a result, the perimeter $P_{n}$ of the Koch snowflake is calculated as follows

$$
P_{n}=N_{n} \cdot L_{n}=\frac{4}{3} N_{n-1} \cdot L_{n-1}=\frac{4}{3} P_{n-1}=\frac{4^{n}}{3^{n-1}} l .
$$

Therefore, if we start our computations from the original triangle from Fig. 9.2, after (1) steps we have the snowflake having the infinite number of segments $N_{\circledast}=3 \cdot 4^{\circledR}$. Each of 
the segments has the infinitesimal length $L_{\oplus}=\frac{1}{3^{\natural}} l$. In order to calculate the perimeter, $P_{\Phi}$, of the snowflake we should multiply the infinite number $N_{\oplus}$ and the infinitesimal number $L_{\oplus}$. Thus, the perimeter is

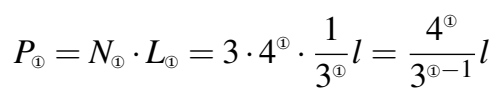

and it is infinite. Analogously, after executing (1)-1 steps we have the infinite perimeter $P_{(\mathbb{1}-1}=\frac{4^{(\mathbb{1}-1}}{3^{(\mathbb{1}-2}} l$. Since the new numeral systems allows us to execute easily arithmetical operations with infinite numbers, we can divide the obtained two infinite numbers, $P_{\varpi}$ and $P_{\mathbb{1}-1}$, one by another and to obtain as the result the finite number that is in a complete agreement with $(9.3)$

$$
\frac{P_{(1)}}{P_{(1)-1}}=\frac{\frac{4^{(1)}}{3^{(-1}} l}{\frac{4^{(1-1}}{3^{(1-2}} l}=\frac{4}{3} .
$$

The difference of the two perimeters can also be calculated easily

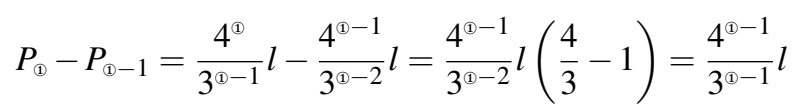

and it turns out to be infinite.

In case the infinite number of steps is $n=0.51$, it follows that the infinite perimeter is $P_{0.5 \oplus}=\frac{4^{0.5 \Phi}}{3^{0.5 \Phi-1}} l$ and the operation of division of two infinite numbers gives us as the result also an infinite number that can be calculated precisely:

$$
\frac{P_{(}}{P_{0.5 \Phi}}=\frac{\frac{4^{\circledR}}{3^{(1-1}} l}{\frac{4^{0.5 \Phi}}{3^{0.5(-1}} l}=\left(\frac{4}{3}\right)^{0.5 \bowtie} \text {. }
$$

Thus we can distinguish now in a precise manner that the infinite perimeter $P_{\circledast}$ is infinitely longer than the infinite perimeter $P_{0.5 \text { ब }}$.

We can also distinguish at infinity the snowflakes having different initial generators. As we have already seen, starting from the original triangle after (1) steps the snowflake has $3 \cdot 4^{\Phi}$ segments and each of them has the infinitesimal length $L_{\circledast}=\frac{1}{3^{\Phi}} l$ and the perimeter of the snowflake is $P_{(}=\frac{4^{\circledR}}{3^{(-1}} l$. If we start from the initial configuration shown at Fig. 9.2, c) and also execute (1) steps then the resulting snowflake will have $N_{\Phi+2}=3 \cdot 4^{\circledR+2}$ segments, each of them will have the infinitesimal length $L_{\mathbb{1}+2}=\frac{1}{3^{\mathbb{1}+2}} l$ and the perimeter of the snowflake will be $P_{\mathbb{1}+2}=\frac{4^{\mathbb{1}+2}}{3^{\mathbb{1}+1}} l$. Thus, this snowflake will have infinitely many more segments than the one started from the original triangle. More precisely, this infinite difference is equal to

$$
N_{\Phi}+2-N_{\Phi}=3 \cdot 4^{\oplus+2}-3 \cdot 4^{\Phi}=3 \cdot 4^{\Phi}\left(4^{2}-1\right)=45 \cdot 4^{\oplus} .
$$

The lengths of the segments in both snowflakes are infinitesimal and, in spite of the fact that their difference is also infinitesimal, it can be calculated precisely as follows

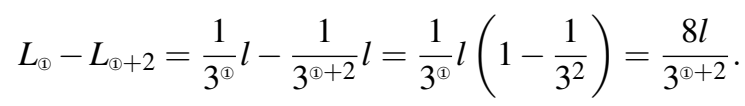


Let us see now what happens with the area of the snowflake at infinity. As it can be seen from Fig. 9.2, at each iteration $n$ a new triangle is added at each side of the snowflake built at iteration $n-1$ and, therefore, the number of new triangles, $T_{n}$, is equal to

$$
T_{n}=N_{n-1}=3 \cdot 4^{n-1} .
$$

The area, $a_{n}$, of each triangle added at $n$-th iteration is $\frac{1}{9}$ of each triangle added during the iteration $n-1$

$$
a_{n}=\frac{a_{n-1}}{9}=\frac{a_{0}}{9^{n}},
$$

where $a_{0}$ is the area of the original triangle (see (9.1)). Therefore, the whole new area added to the snowflake is

$$
T_{n} a_{n}=3 \cdot 4^{n-1} \cdot \frac{a_{0}}{9^{n}}=\frac{3}{4} \cdot\left(\frac{4}{9}\right)^{n} \cdot a_{0}=\frac{a_{0}}{3} \cdot\left(\frac{4}{9}\right)^{n-1}
$$

and the complete area, $A_{n}$, of the snowflake at the $n$-th iteration is

$$
\begin{aligned}
& A_{n}=a_{0}+\sum_{i=1}^{n} T_{n} a_{n}=a_{0}\left(1+\frac{1}{3} \sum_{i=1}^{n}\left(\frac{4}{9}\right)^{i-1}\right)=a_{0}\left(1+\frac{1}{3} \sum_{i=0}^{n-1}\left(\frac{4}{9}\right)^{i}\right)= \\
& a_{0}\left(1+\frac{1}{3} \cdot \frac{1-\left(\frac{4}{9}\right)^{n}}{1-\frac{4}{9}}\right)=a_{0}\left(1+\frac{3}{5}\left(1-\left(\frac{4}{9}\right)^{n}\right)\right)=\frac{a_{0}}{5}\left(8-3\left(\frac{4}{9}\right)^{n}\right) .
\end{aligned}
$$

Traditionally, the limit of the area is considered and the result

$$
\lim _{n \rightarrow \infty} A_{n}=\frac{8}{5} a_{0}
$$

is obtained. Thanks to (1)-based numerals we are able now to work with infinitesimals easily and to observe the infinitesimal difference of the areas of the snowflakes at infinity. For example, after (1)-1 and (1) iterations the difference between the areas $A_{\varpi-1}$ and $A_{\circledast}$ is

$$
A_{\circledast}-A_{\Phi-1}=\frac{a_{0}}{5}\left(8-3\left(\frac{4}{9}\right)^{\Phi}\right)-\frac{a_{0}}{5}\left(8-3\left(\frac{4}{9}\right)^{\mathbb{\Phi}-1}\right)=\frac{a_{0}}{3}\left(\frac{4}{9}\right)^{\Phi-1}>0 .
$$

This number is an infinitesimal and it perfectly corresponds to the general formula (9.4).

\section{A brief conclusion}

In this survey, a recent computational methodology has been described. It allows one to express, by a finite number of symbols, not only finite numbers but infinities and infinitesimals, as well, and to execute numerical (not symbolic) computations with all of them on a new kind of supercomputer. It should be stressed that traditional approaches dealing with infinity and the new methodology do not contradict one another. They just describe mathematical objects with different accuracies. Several theoretical and applied problems 
where the new methodology helps a lot have been discussed. Numerous examples and illustrations have been provided.

It has been emphasized that the philosophical triad - researcher, object of investigation, and tools used to observe the object - existing in such natural sciences as Physics and Chemistry, exists in Mathematics and Computer Science, too. In natural sciences, the instrument used to observe the object influences the results of observation. The same happens in Mathematics and Computer Science, where numeral systems used to express numbers are among the instruments of observations used by mathematicians. The usage of powerful numeral systems yields more precise results in Mathematics, in the same way as the usage of a good microscope gives the possibility to obtain more precise results in Physics. When a mathematician chooses a numeral system (an instrument), in this moment he/she chooses both a set of numbers that can be observed through the numerals available in the chosen numeral system and the accuracy of results that can be obtained during computations.

The analysis performed in this study shows that the traditional mathematical language using symbols $\infty, \omega, \boldsymbol{\aleph}_{0}, \boldsymbol{\aleph}_{1}$, etc. very often does not possess a sufficiently high accuracy when one deals with problems having their interesting properties at infinity. This lack of accuracy can lead to paradoxes and problems that are considered to be very hard in traditional Mathematics. It has been emphasized that their resistance to solution is often a consequence of the weakness of numeral systems used traditionally. Numerous theoretical and applied problems considered here show that the (1)-based numeral system can help avoid difficulties and paradoxes on several occasions and it gives rise not only to powerful computational and theoretical techniques but also to the possibility to work with mathematical objects that are invisible if one uses traditional tools.

In particular, the Continuum Hypothesis and the Riemann Hypothesis were studied. It has been shown that the hardness of both problems is not related to their nature but is induced by the weakness of traditional numeral systems used to formulate and study the problems. The application of the (1)-based numeral system has allowed us to avoid traditional difficulties and to observe mathematical objects involved in the two problems in a new light and with a higher accuracy. The effect of employing the (1)-based methodology in the study of the above Hypotheses is comparable to the dissolution of computational problems posed in Roman numerals (e.g., X - X) once a positional system capable of expressing zero is adopted.

In conclusion, several pure mathematical and numerical applications discussed here show that the introduction of the (1)-based methodology gives rise to a more accurate description of a variety of mathematical objects, leads to the construction of new powerful numerical methods, and provides answers to several problems that look exceptionally challenging when traditional mathematical tools are applied.

\section{References}

[1] R.A. Adams. Single variable calculus. Pearson Education Canada, Ontario, 5 edition, 2003. 
[2] P. Amodio, F. Iavernaro, F. Mazzia, M.S. Mukhametzhanov, and Ya.D. Sergeyev. A generalized Taylor method of order three for the solution of initial value problems in standard and infinity floating-point arithmetic. Mathematics and Computers in Simulation, 141:24-39, 2017.

[3] J. Bagaria and M. Magidor. Group radicals and strongly compact cardinals. Transactions of the American Mathematical Society, 366(4):1857-1877, 2014.

[4] M. Balazard and O.V. Castan. Sur l'infimum des parties réelles des zéros des sommes partielles de la fonction zêta de Riemann. http://front.math. ucdavis.edu/0902.0923, 2009.

[5] R. Barrio. Performance of the Taylor series method for ODEs/DAEs. Applied Mathematics and Computation, 163(2):525-545, 2005. cited By 47.

[6] V. Benci and M. Di Nasso. Numerosities of labeled sets: a new way of counting. Advances in Mathematics, 173:50-67, 2003.

[7] M. Berz. Automatic differentiation as nonarchimedean analysis. In Computer Arithmetic and Enclosure Methods, pages 439-450. Elsevier, Amsterdam, 1992.

[8] W.H. Beyer, editor. CRC standard mathematical tables. CRC Press, West Palm Beach, Fla., 1978.

[9] C. Bischof and M. Bücker. Computing derivatives of computer programs. In Modern Methods and Algorithms of Quantum Chemistry Proceedings, NIC Series, vol. 3, pages 315-327. John von Neumann Institute for Computing, Jülich, 2 edition, 2000.

[10] E. Bombieri. The mathematical infinity. In M. Heller and W. Hugh Woodin, editors, Infinity: New Research Frontiers, pages 55-75. Cambridge University Press, Cambridge, 2011.

[11] P. Borwein, G. Fee, R. Ferguson, and A. van der Waal. Zeros of partial summs of the Riemann zeta function. Experiment. Math., 16(1):21-40, 2007.

[12] L. Brugnano and D. Trigiante. Solving Differential Problems by Multistep Initial and Boundary Value Methods. Gordon and Breach Science Publ., Amsterdam, 1998.

[13] J.C. Butcher. Numerical methods for ordinary differential equations. John Wiley \& Sons, Chichester, 2 edition, 2003.

[14] B. Butterworth, R. Reeve, F. Reynolds, and D. Lloyd. Numerical thought with and without words: Evidence from indigenous Australian children. Proceedings of the National Academy of Sciences of the United States of America, 105(35):1317913184, 2008.

[15] F. Caldarola. The Sierpinski curve viewed by numerical computations with infinities and infinitesimals. Applied Mathematics and Computation, 318:321-328, 2018.

[16] G. Cantor. Contributions to the founding of the theory of transfinite numbers. Dover Publications, New York, 1955. 
[17] J.B. Carroll, editor. Language, Thought, and Reality: Selected Writings of Benjamin Lee Whorf. MIT Press, 1956.

[18] J.R. Cash and S. Considine. An mebdf code for stiff initial value problems. ACM Trans. Math. Software, 18(2):142-155, 1992.

[19] A.L. Cauchy. Le Calcul infinitésimal. Paris, 1823.

[20] M. Cococcioni, M. Pappalardo, and Ya.D. Sergeyev. Lexicographic multi-objective linear programming using grossone methodology: Theory and algorithm. Applied Mathematics and Computation, 318:298-311, 2018.

[21] J.S. Cohen. Computer Algebra and Symbolic Computation: Mathematical Methods. A K Peters, Ltd., Wellesley, MA, 1966.

[22] P.J. Cohen. Set Theory and the Continuum Hypothesis. Benjamin, New York, 1966.

[23] M. Colyvan. An Introduction to the Philosophy of Mathematics. Cambridge University Press, Cambridge, 2012.

[24] J. Brian Conrey. The Riemann hypothesis. Notices Amer. Math. Soc., 50(3):341353, 2003.

[25] J.H. Conway and R.K. Guy. The Book of Numbers. Springer-Verlag, New York, 1996.

[26] G. Corliss, C. Faure, A. Griewank, L. Hascoet, and U. Naumann, editors. Automatic Differentiation of Algorithms: From Simulation to Optimization. Springer-Verlag, New York, 2002.

[27] L. Corry. Numbers. Oxford University Press, Oxford, 2015.

[28] J. d'Alembert. Différentiel. Encyclopédie, ou dictionnaire raisonné des sciences, des arts et des métiers, 4, 1754.

[29] L. D'Alotto. Cellular automata using infinite computations. Applied Mathematics and Computation, 218(16):8077-8082, 2012.

[30] L. D'Alotto. A classification of two-dimensional cellular automata using infinite computations. Indian Journal of Mathematics, 55:143-158, 2013.

[31] L. D'Alotto. A classification of one-dimensional cellular automata using infinite computations. Applied Mathematics and Computation, 255:15-24, 2015.

[32] S. De Cosmis and R. De Leone. The use of grossone in mathematical programming and operations research. Applied Mathematics and Computation, 218(16):80298038, 2012.

[33] R. De Leone. Nonlinear programming and grossone: Quadratic programming and the role of constraint qualifications. Applied Mathematics and Computation, 318:290-297, 2018.

[34] R. De Leone, G. Fasano, and Ya.D. Sergeyev. Planar methods and grossone for the conjugate gradient breakdown in nonlinear programming. Computational Optimization and Applications, in press. 
[35] R.L. Devaney. An Introduction to Chaotic Dynamical Systems. Westview Press Inc., New York, 2003.

[36] H.M. Edwards. Riemann's zeta function. Dover Publications, New York, 2001.

[37] L. Euler. De seriebus quibusdam considerationes. Commentarii academiae scientarium Petropolitanae, 12:53-96, Opera omnia, Series prima XIV, pp. 407-461, 1740.

[38] L. Euler. De seriebus divergentibus. Novi commentarii academiae scientarium Petropolitanae, 5:205-237, Opera omnia, Series prima XIV, pp. 585-617, 17541755.

[39] L. Euler. Remarques sur un beau rapport entre les series des puissances tant directes que reciproques. Memoires de lacademie des sciences de Berlin, 17:83-106. Opera omnia, Series prima XV, pp. 70-90, 1761.

[40] L. Euler. An introduction to the analysis of the infinite (translated by John D. Blanton). Springer-Verlag, New York, 1988.

[41] K. Falconer. Fractal Geometry: Mathematical foundations and applications. John Wiley \& Sons, Chichester, 1995.

[42] G. Gamow. One, Two, Three...Infinity. Viking Press, New York, 1961.

[43] M. Gaudioso, G. Giallombardo, and M. S. Mukhametzhanov. Numerical infinitesimals in a variable metric method for convex nonsmooth optimization. Applied Mathematics and Computation, 318:312-320, 2018.

[44] K. Gödel. The Consistency of the Continuum-Hypothesis. Princeton University Press, Princeton, 1940.

[45] P. Gordon. Numerical cognition without words: Evidence from Amazonia. Science, 306(15 October):496-499, 2004.

[46] N. Greenberg, D. Hirschfeldt, J.D. Hamkins, and R. Miller, editors. Effective Mathematics of the Uncountable. Lecture Notes in Logic. Cambridge University Press, Cambridge, 2001.

[47] D.F. Griffiths and D.J. Higham. Numerical methods for Ordinary differential equations. Initial Value Problems. Springer Undergraduate Mathematics Series, 271, 2010.

[48] E. Hairer, S. P. Nørsett, and G. Wanner. Solving ordinary differential equations I: Nonstiff problems. Springer-Verlag, New York, 2 edition, 1993.

[49] G.H. Hardy. Orders of infinity. Cambridge University Press, Cambridge, 1910.

[50] H.M. Hastings and G. Sugihara. Fractals: A user's guide for the natural sciences. Oxford University Press, Oxford, 1994.

[51] M. Heller and W. Hugh Woodin, editors. Infinity: New Research Frontiers. Cambridge University Press, Cambridge, 2011.

[52] P. Henrici. Applied and computational complex analysis, volume 1. John Wiley \& Sons, Chichester, 1997. 
[53] D. Hilbert. Mathematical problems: Lecture delivered before the International Congress of Mathematicians at Paris in 1900. Bulletin of the American Mathematical Society, 8:437-479, 1902.

[54] F. Iavernaro and F. Mazzia. Solving ordinary differential equations by generalized adams methods: properties and implementation techniques. Appl. Numer. Math., 28(2-4):107-126, 1998.

[55] H. Isermann. Linear lexicographic optimization. OR Spektrum, 4:223-228, 1982.

[56] D.I. Iudin, Ya.D. Sergeyev, and M. Hayakawa. Interpretation of percolation in terms of infinity computations. Applied Mathematics and Computation, 218(16):8099-8111, 2012.

[57] D.I. Iudin, Ya.D. Sergeyev, and M. Hayakawa. Infinity computations in cellular automaton forest-fire model. Communications in Nonlinear Science and Numerical Simulation, 20(3):861-870, 2015.

[58] A. Kanamori. The mathematical development of set theory from Cantor to Cohen. Bull. Symbolic Logic, 2(1):1-71, 1996.

[59] A. Kanamori. The Higher Infinite: Large cardinals in set theory from their beginnings. Springer, Berlin Heidelberg, 2 edition, 2003.

[60] K. Knopp. Theory and Application of Infinite Series. Dover Publications, New York, 1990.

[61] H. von Koch. Sur une courbe continue sans tangente, obtenue par une construction géométrique élémentaire. Archiv för Matemat., Astron. och Fys., 1:681-702, 1904.

[62] G.C. Leder. Mathematics for all? The case for and against national testing. In S.J. Cho, editor, The Proceedings of the 12th International Congress on Mathematical Education: Intellectual and Attitudinal Chalenges, pages 189-207. Springer, New York, 2015.

[63] G.W. Leibniz and J.M. Child. The Early Mathematical Manuscripts of Leibniz. Dover Publications, New York, 2005.

[64] T. Levi-Civita. Sui numeri transfiniti. Rend. Acc. Lincei, Series 5a, 113:7-91, 1898.

[65] G. Lolli. Infinitesimals and infinites in the history of mathematics: A brief survey. Applied Mathematics and Computation, 218(16):7979-7988, 2012.

[66] G. Lolli. Metamathematical investigations on the theory of grossone. Applied Mathematics and Computation, 255:3-14, 2015.

[67] M. Margenstern. Using grossone to count the number of elements of infinite sets and the connection with bijections. p-Adic Numbers, Ultrametric Analysis and Applications, 3(3):196-204, 2011.

[68] M. Margenstern. An application of grossone to the study of a family of tilings of the hyperbolic plane. Applied Mathematics and Computation, 218(16):8005-8018, 2012. 
[69] M. Margenstern. Fibonacci words, hyperbolic tilings and grossone. Communications in Nonlinear Science and Numerical Simulation, 21(1-3):3-11, 2015.

[70] M. Margenstern. Infinigons of the hyperbolic plane and grossone. Applied Mathematics and Computation, 278:45-53, 2016.

[71] A.A. Markov Jr. and N.M. Nagorny. Theory of Algorithms. FAZIS, Moscow, second edition, 1996.

[72] J.P. Mayberry. The Foundations of Mathematics in the Theory of Sets. Cambridge University Press, Cambridge, 2001.

[73] F. Mazzia and A.M. Nagy. A new mesh selection strategy with stiffness detection for explicit Runge-Kutta methods. Applied Mathematics and Computation, 255:125-134, 2015.

[74] F. Mazzia, Ya.D. Sergeyev, F. Iavernaro, P. Amodio, and M.S. Mukhametzhanov. Numerical methods for solving ODEs on the Infinity Computer. In Sergeyev Ya.D., Kvasov D.E., Dell'Accio F., and Mukhametzhanov M.S., editors, Proc. of the 2nd Intern. Conf. "Numerical Computations: Theory and Algorithms", volume 1776, page 090033. AIP Publishing, New York, 2016.

[75] F. Montagna, G. Simi, and A. Sorbi. Taking the Pirahã seriously. Communications in Nonlinear Science and Numerical Simulation, 21(1-3):52-69, 2015.

[76] J.M. Muller. Elementary functions: algorithms and implementation. Birkhäuser, Boston, 2006.

[77] I. Newton. Method of Fluxions. 1671.

[78] H.-O. Peitgen, H. Jürgens, and D. Saupe. Chaos and Fractals. Springer-Verlag, New York, 1992.

[79] P. Pica, C. Lemer, V. Izard, and S. Dehaene. Exact and approximate arithmetic in an amazonian indigene group. Science, 306(15 October):499-503, 2004.

[80] L. Pourkarimi and M. Zarepisheh. A dual-based algorithm for solving lexicographic multiple objective programs. European Journal of Operational Research, 176:1348-1356, 2007.

[81] D. Rizza. Supertasks and numeral systems. In Sergeyev Ya.D., Kvasov D.E., Dell'Accio F., and Mukhametzhanov M.S., editors, Proc. of the 2nd Intern. Conf. "Numerical Computations: Theory and Algorithms", volume 1776, page 090005. AIP Publishing, New York, 2016.

[82] A. Robinson. Non-standard Analysis. Princeton Univ. Press, Princeton, 1996.

[83] H. Sagan. Space-Filling Curves. Springer, New York, 1994.

[84] E. Sapir. Selected Writings of Edward Sapir in Language, Culture and Personality. University of California Press, Princeton, 1958.

[85] Ya. D. Sergeyev, R. G. Strongin, and D. Lera. Introduction to Global Optimization Exploiting Space-Filling Curves. Springer, New York, 2013.

[86] Ya.D. Sergeyev. Arithmetic of Infinity. Edizioni Orizzonti Meridionali, CS, 2003, 2nd ed. 2013. 
[87] Ya.D. Sergeyev. Blinking fractals and their quantitative analysis using infinite and infinitesimal numbers. Chaos, Solitons \& Fractals, 33(1):50-75, 2007.

[88] Ya.D. Sergeyev. A new applied approach for executing computations with infinite and infinitesimal quantities. Informatica, 19(4):567-596, 2008.

[89] Ya.D. Sergeyev. Evaluating the exact infinitesimal values of area of Sierpinski's carpet and volume of Menger's sponge. Chaos, Solitons \& Fractals, 42(5):30423046, 2009.

[90] Ya.D. Sergeyev. Numerical computations and mathematical modelling with infinite and infinitesimal numbers. Journal of Applied Mathematics and Computing, 29:177-195, 2009.

[91] Ya.D. Sergeyev. Numerical point of view on Calculus for functions assuming finite, infinite, and infinitesimal values over finite, infinite, and infinitesimal domains. Nonlinear Analysis Series A: Theory, Methods \& Applications, 71(12):e1688e1707, 2009.

[92] Ya.D. Sergeyev. Computer system for storing infinite, infinitesimal, and finite quantities and executing arithmetical operations with them. USA patent 7,860,914, 2010.

[93] Ya.D. Sergeyev. Counting systems and the First Hilbert problem. Nonlinear Analysis Series A: Theory, Methods \& Applications, 72(3-4):1701-1708, 2010.

[94] Ya.D. Sergeyev. Lagrange Lecture: Methodology of numerical computations with infinities and infinitesimals. Rendiconti del Seminario Matematico dell'Università e del Politecnico di Torino, 68(2):95-113, 2010.

[95] Ya.D. Sergeyev. Higher order numerical differentiation on the Infinity Computer. Optimization Letters, 5(4):575-585, 2011.

[96] Ya.D. Sergeyev. On accuracy of mathematical languages used to deal with the Riemann zeta function and the Dirichlet eta function. $p$-Adic Numbers, Ultrametric Analysis and Applications, 3(2):129-148, 2011.

[97] Ya.D. Sergeyev. Using blinking fractals for mathematical modelling of processes of growth in biological systems. Informatica, 22(4):559-576, 2011.

[98] Ya.D. Sergeyev. Solving ordinary differential equations by working with infinitesimals numerically on the Infinity Computer. Applied Mathematics and Computation, 219(22):10668-10681, 2013.

[99] Ya.D. Sergeyev. Computations with grossone-based infinities. In Calude C.S. and Dinneen M.J., editors, Unconventional Computation and Natural Computation: Proc. of the 14th International Conference UCNC 2015, volume LNCS 9252, pages 89-106. Springer, New York, 2015.

[100] Ya.D. Sergeyev. The Olympic medals ranks, lexicographic ordering, and numerical infinities. The Mathematical Intelligencer, 37(2):4-8, 2015.

[101] Ya.D. Sergeyev. Un semplice modo per trattare le grandezze infinite ed infinitesime. Matematica nella Società e nella Cultura: Rivista della Unione Matematica Italiana, 8(1):111-147, 2015. 
[102] Ya.D. Sergeyev. The exact (up to infinitesimals) infinite perimeter of the Koch snowflake and its finite area. Communications in Nonlinear Science and Numerical Simulation, 31(1-3):21-29, 2016.

[103] Ya.D. Sergeyev and A. Garro. Observability of Turing machines: A refinement of the theory of computation. Informatica, 21(3):425-454, 2010.

[104] Ya.D. Sergeyev and A. Garro. Single-tape and multi-tape Turing machines through the lens of the Grossone methodology. Journal of Supercomputing, 65(2):645-663, 2013.

[105] Ya.D. Sergeyev, M.S. Mukhametzhanov, F. Mazzia, F. Iavernaro, and P. Amodio. Numerical methods for solving initial value problems on the Infinity Computer. International Journal of Unconventional Computing, 12(1):3-23, 2016.

[106] S. Shelah. Cardinal Arithmetic, volume 29 of Oxford Logic Guides. Oxford University Press, 1994.

[107] H.D. Sherali and A.L. Soyster. Preemptive and nonpreemptive multi-objective programming: Relationship and counterexamples. Journal of Optimization Theory and Applications, 39(2):173-186, 1983.

[108] R. Spira. Zeros of sections of the zeta function. I. Mathematics of Computation, 20(96):542-550, 1966.

[109] I.P. Stanimirovic. Compendious lexicographic method for multi-objective optimization. Facta universitatis - series: Mathematics and Informatics, 27(1):55-66, 2012.

[110] M.C. Vita, S. De Bartolo, C. Fallico, and M. Veltri. Usage of infinitesimals in the Menger's Sponge model of porosity. Applied Mathematics and Computation, 218(16):8187-8196, 2012.

[111] P. Vopěnka and P. Hájek. The Theory of Semisets. Academia Praha/North Holland Publishing Company, Praha, 1972.

[112] J. Wallis. Arithmetica infinitorum. 1656.

[113] W. H. Woodin. The Continuum Hypothesis, Part I. Notices of the AMS, 48(6):567576, 2001.

[114] A. Zhigljavsky. Computing sums of conditionally convergent and divergent series using the concept of grossone. Applied Mathematics and Computation, 218(16):8064-8076, 2012.

[115] A. Zhigljavsky and V. Kornikov. Classical areas of mathematics where the concept of grossone could be useful. In Sergeyev Ya.D., Kvasov D.E., Dell'Accio F., and Mukhametzhanov M.S., editors, Proc. of the 2nd Intern. Conf. "Numerical Computations: Theory and Algorithms”, volume 1776, page 020004. AIP Publishing, New York, 2016.

[116] A. Žilinskas. On strong homogeneity of two global optimization algorithms based on statistical models of multimodal objective functions. Applied Mathematics and Computation, 218(16):8131-8136, 2012. 
Received 2017-07-14; revised 2017-08-25 